\title{
Sulfur and Selenium Ylide Bond Enthalpies
}

Stacey A. Stoffregen, Ryan D. McCulla, Robert Wilson, Samuel Cercone, Jennifer Miller and

William S. Jenks*

Department of Chemistry, Iowa State University, Ames, IA 50011-3111

\section{Supporting Information}

Coordinates and Absolute Energies ..............................................................................2

Computed BDEs as a function of basis set .................................................................75

Table S1. Computational and experimental BDEs for sulfoxides (1-4) in $\mathrm{kcal} / \mathrm{mol}$............75

Table S2. Computational and experimental BDEs for sulfoxides (5-9) in $\mathrm{kcal} / \mathrm{mol}$.............76

Table S3. BDEs for 1-4 from isodesmic reactions at different basis sets, in $\mathrm{kcal} / \mathrm{mol}$..........77

Table S4. BDEs for sulfilimines 10-15 as a function of basis set....................................79

Table S5. Calculated BDEs for sulfonium carbenes 19-23 in kcal $/ \mathrm{mol}$.............................8 80

Table S6. Computed BDEs for selenoxides 27-32, in $\mathrm{kcal} / \mathrm{mol}$................................... 81 


\section{Coordinates and Absolute Energies}

\section{$O\left({ }^{3} \mathrm{P}\right)$}

$\operatorname{MP} 2 / 6-31 G(d, p)$

$0.0 \quad .00000000000 \quad .0000000000 \quad .0000000000$

Energy

ZPE Correction

298.15K Temperature Correction

$-46986.7678 \mathrm{KCAL} / \mathrm{MOL}$

$0.0000 \mathrm{KCAL} / \mathrm{MOL}$

$1.481 \mathrm{KCAL} / \mathrm{MOL}$

\begin{tabular}{lcc}
\hline Basis Set & MP2 (kcal/mol) & B3LYP (kcal/mol) \\
\hline $6-31 G(d)$ & -46986.76779 & -47081.58153 \\
$6-31 G(2 d)$ & -46999.70479 & -47083.33631 \\
$6-31 G(d, p)$ & -46986.76779 & -47081.58153 \\
$6-31+G(d)$ & -46989.90189 & -47085.87546 \\
$6-31+G(2 d, p)$ & -47002.75640 & -47087.68341 \\
$6-311 G(d)$ & -47013.53427 & -47097.52784 \\
$6-311 G(3 d f, 2 p)$ & -47034.84814 & -47098.62731 \\
$6-311++G(d)$ & -47015.47389 & -47100.23089 \\
$6-311++G(3 d f, 2 p)$ & -47036.97187 & -47101.60628 \\
\hline$G 3(298.15 \mathrm{~K})$ & -47081.80861 \\
G3 (0K) & -47082.69716 \\
\hline
\end{tabular}

TOTAL MULLIKEN AND LOWDIN ATOMIC POPULATIONS

$\begin{array}{lllll}\text { ATOM } & \text { MULL.POP } & \text { CHARGE } & \text { LOW.POP. } & \text { CHARGE } \\ 10 & 8.000000 & .000000 & 8.000000 & .000000\end{array}$




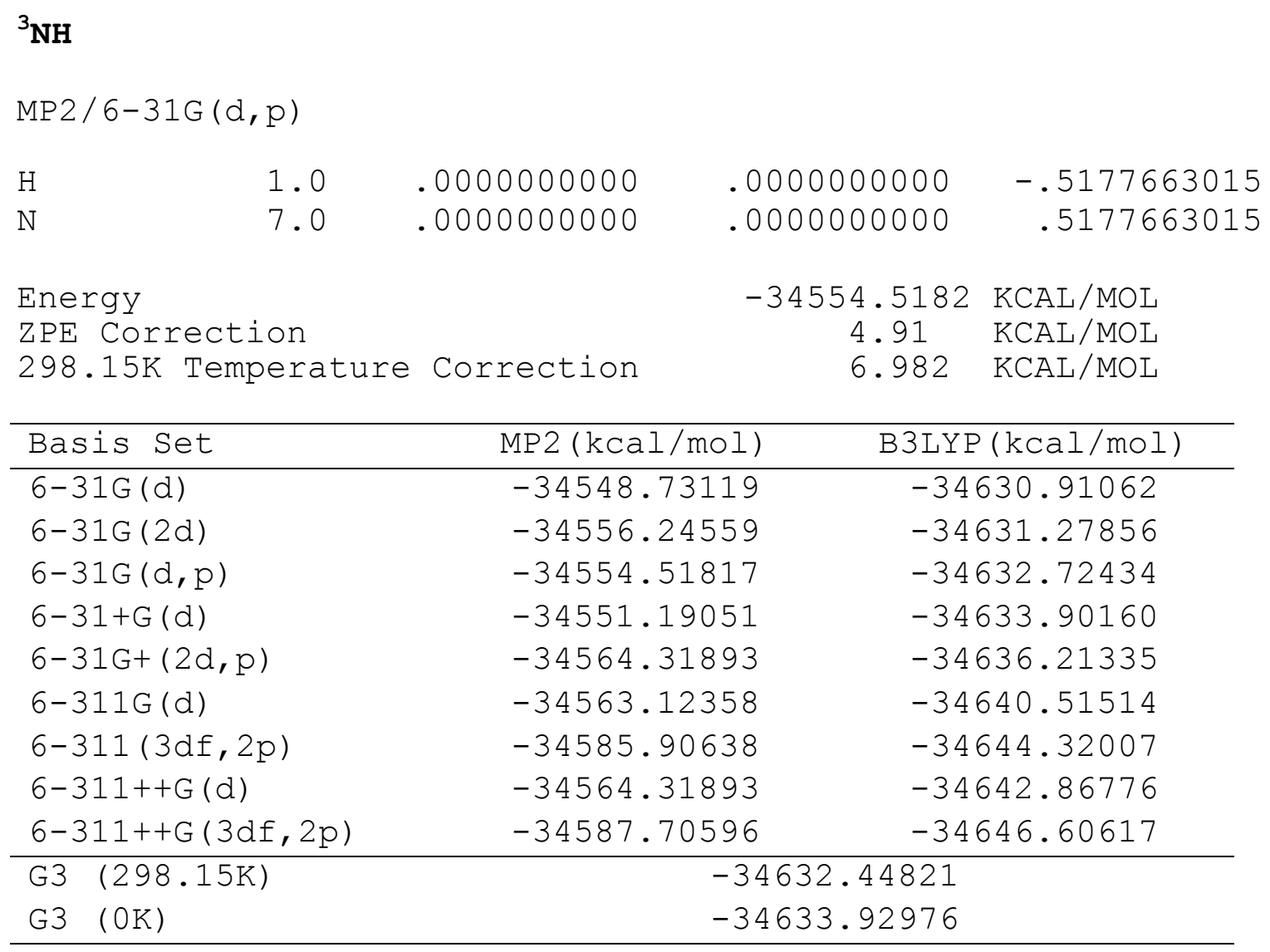

TOTAL MULLIKEN AND LOWDIN ATOMIC POPULATIONS

$\begin{array}{lcrrr}\text { ATOM } & \text { MULL.POP } & \text { CHARGE } & \text { LOW.POP. } & \text { CHARGE } \\ 1 \mathrm{H} & .752252 & .247748 & .841911 & .158089 \\ 2 \mathrm{~N} & 7.247748 & -.247748 & 7.158089 & -.158089\end{array}$

${ }^{3} \mathrm{CH}_{2}$

$\operatorname{MP} 2 / 6-31 G(d, p)$

$\begin{array}{lrrrr}\mathrm{H} & 1.0 & .0000000000 & -.9785778959 & .7429948987 \\ \mathrm{H} & 1.0 & .0000000000 & .9785778959 & .7429948987 \\ \mathrm{C} & 6.0 & .0000000000 & .0000000000 & .3000882006\end{array}$

Energy

ZPE Correction

298.15K Temperature Correction

$-24483.642 \mathrm{KCAL} / \mathrm{MOL}$

$11.36 \mathrm{KCAL} / \mathrm{MOL}$

$13.739 \mathrm{KCAL} / \mathrm{MOL}$ 


\begin{tabular}{lcc}
\hline Basis Set & MP2 $(\mathrm{kcal} / \mathrm{mol})$ & B3LYP $(\mathrm{kcal} / \mathrm{mol})$ \\
\hline $6-31 \mathrm{G}(\mathrm{d})$ & -24473.6073 & -24547.83779 \\
$6-31 \mathrm{G}(2 \mathrm{~d})$ & -24478.25298 & -24547.54653 \\
$6-31 \mathrm{G}(\mathrm{d}, \mathrm{p})$ & -24483.64159 & -24550.00374 \\
$6-31+\mathrm{G}(\mathrm{d})$ & -24475.88709 & -24528.29615 \\
$6-311 \mathrm{G}(\mathrm{d})$ & -24483.9349 & -24554.81581 \\
$6-311 \mathrm{G}(3 \mathrm{df}, 2 \mathrm{p})$ & -24504.80837 & -24558.55652 \\
$6-311++\mathrm{G}(\mathrm{d})$ & -24484.62501 & -24555.73821 \\
$6-311++\mathrm{G}(3 \mathrm{df}, 2 \mathrm{p})$ & -24505.58167 & -24559.58917 \\
\hline G3 (298.15K) & \multicolumn{2}{c}{-24545.23312} \\
G3 (0K) & \multicolumn{2}{c}{-24547.02654} \\
\hline
\end{tabular}

TOTAL MULLIKEN AND LOWDIN ATOMIC POPULATIONS

\begin{tabular}{lrrrr} 
ATOM & MULL.POP & CHARGE & LOW.POP. & \multicolumn{1}{c}{ CHARGE } \\
$1 \mathrm{H}$ & .863418 & .136582 & .880810 & .119190 \\
$2 \mathrm{H}$ & .863418 & .136582 & .880810 & .119190 \\
$3 \mathrm{C}$ & 6.273164 & -.273164 & 6.238380 & -.238380
\end{tabular}

\section{${ }^{1}$ formyl nitrene}

MP2 / 6-31G ( d, p)

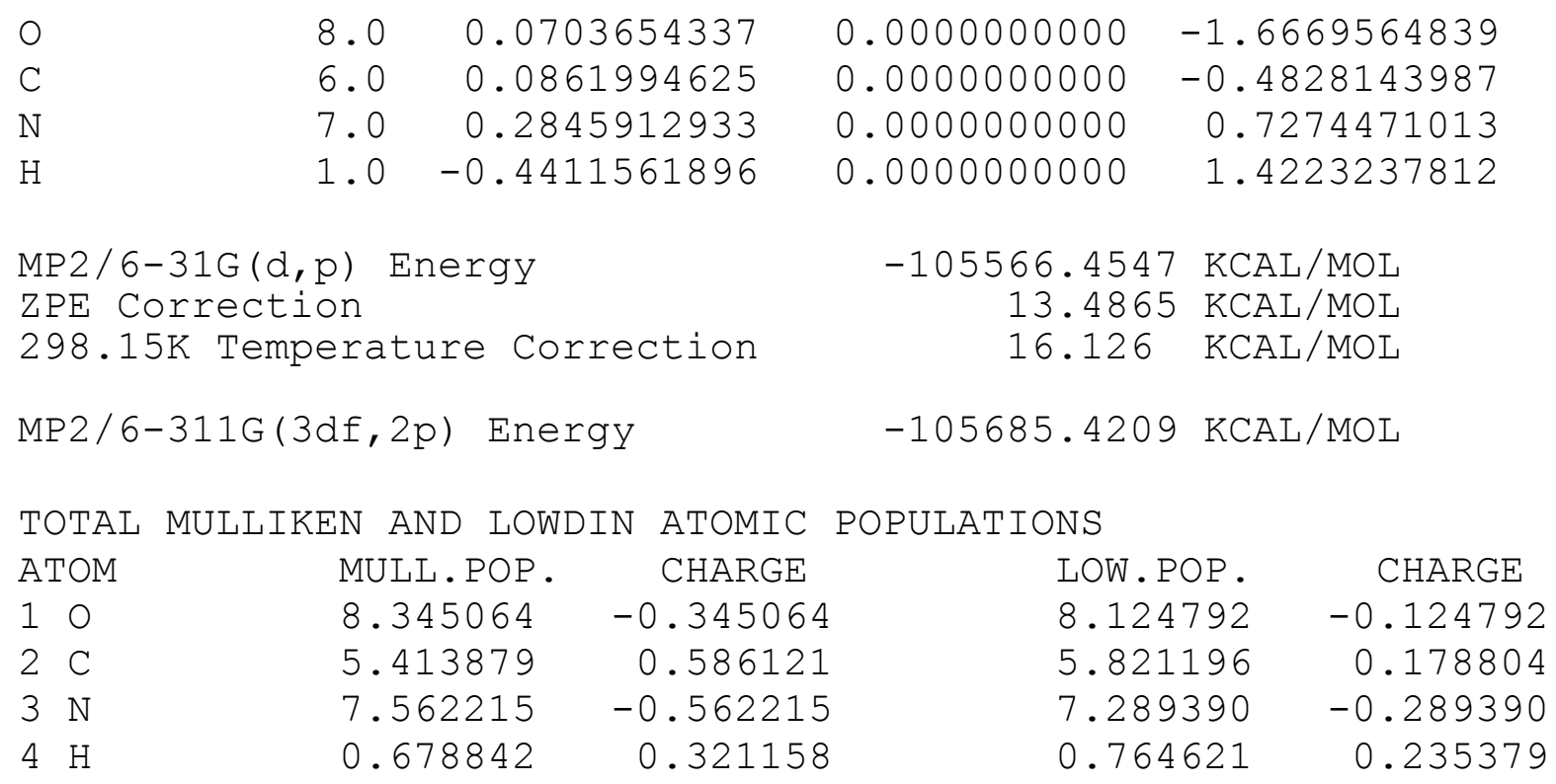




\section{${ }^{3}$ formyl nitrene}

$\operatorname{MP} 2 / 6-31 G(d, p)$

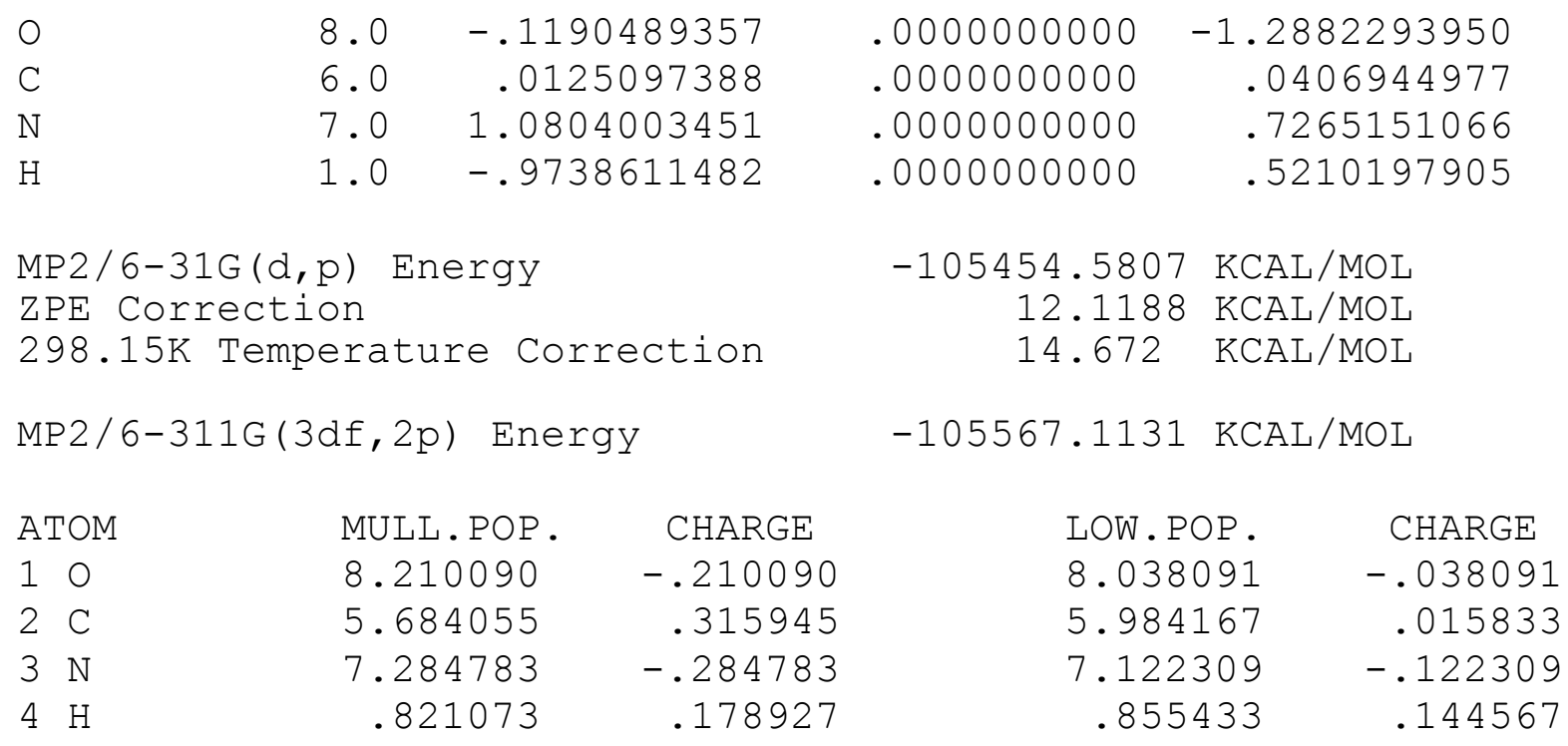

\section{${ }^{3}$ methylsulfonyl nitrene}

$\operatorname{MP} 2 / 6-31 G(d, p)$

\begin{tabular}{|c|c|c|c|c|c|}
\hline 0 & 8.0 & \multicolumn{2}{|c|}{0.8772741700} & 1.0237010808 & -1.4029063756 \\
\hline S & 16.0 & \multicolumn{2}{|c|}{-0.0915960667} & 0.7881695797 & -0.3353279792 \\
\hline O & 8.0 & \multicolumn{2}{|c|}{-1.5022662333} & 1.1465927975 & -0.4593311495 \\
\hline $\mathrm{N}$ & 7.0 & \multicolumn{2}{|c|}{0.5098252861} & 1.6148141747 & 1.0691430719 \\
\hline C & 6.0 & \multicolumn{2}{|c|}{0.0379838284} & -0.8973142132 & 0.2146715634 \\
\hline $\mathrm{H}$ & 1.0 & \multicolumn{2}{|c|}{1.0779627302} & -1.1241674706 & 0.4304326206 \\
\hline $\mathrm{H}$ & 1.0 & \multicolumn{2}{|c|}{-0.3191546113} & -1.5146636779 & -0.6063754819 \\
\hline $\mathrm{H}$ & 1.0 & \multicolumn{2}{|c|}{-0.5900291035} & -1.0371322179 & 1.0896937313 \\
\hline \multicolumn{4}{|c|}{$\begin{array}{l}\text { MP2/6-31G }(\mathrm{d}, \mathrm{p}) \text { Energy } \\
\text { ZPE Correction } \\
\text { 298.15K Temperature Correction } \\
\text { MP2/6-311G(3df,2p) Energy }\end{array}$} & $\begin{array}{rl}-402798.0171 & \mathrm{KC} \\
31.8022 & \mathrm{KC} \\
35.838 & \mathrm{KC} \\
-403041.2652 & \mathrm{KC}\end{array}$ & $\begin{array}{l}\mathrm{KCAL} / \mathrm{MOL} \\
\mathrm{KCAL} / \mathrm{MOL} \\
\mathrm{KCAL} / \mathrm{MOL} \\
\mathrm{KCAL} / \mathrm{MOL}\end{array}$ \\
\hline \multicolumn{6}{|c|}{ TOTAL MULLIKEN AND LOW } \\
\hline ATOM & & MULL. POP. & CHARGE & LOW. POP. & CHARGE \\
\hline 10 & & 8.390744 & -.390744 & 8.364159 & -.364159 \\
\hline $2 \mathrm{~s}$ & & 14.798581 & 1.201419 & 14.707160 & 1.292840 \\
\hline 30 & & 8.453931 & -.453931 & 8.517116 & -.517116 \\
\hline $4 \mathrm{~N}$ & & 7.388824 & -.388824 & 7.426737 & -.426737 \\
\hline $5 \mathrm{C}$ & & 6.537977 & -.537977 & 6.439010 & -.439010 \\
\hline $6 \mathrm{H}$ & & .802752 & .197248 & .847755 & .152245 \\
\hline $7 \mathrm{H}$ & & .810567 & .189433 & .847340 & .152660 \\
\hline $8 \mathrm{H}$ & & .816624 & .183376 & .850724 & .149276 \\
\hline
\end{tabular}


$\mathrm{SH}_{2}$

$\operatorname{MP} 2 / 6-31 G(d, p)$

$\begin{array}{rrrrr}\mathrm{H} & 1.0 & .0000000000 & .9638021397 & .3055777504 \\ \mathrm{~S} & 16.0 & .0000000000 & .0000000001 & -.6111555020 \\ \mathrm{H} & 1.0 & .0000000000 & -.9638021398 & . .3055777505\end{array}$

MP2 / 6-31G (d,p) Energy

$-250257.32467 \mathrm{KCAL} / \mathrm{MOL}$

$9.95 \mathrm{KCAL} / \mathrm{MOL}$

ZPE Correction

298.15K Temperature Correction

$12.331 \mathrm{KCAL} / \mathrm{MOL}$

\begin{tabular}{lcc}
\hline Basis Set & MP2 $(\mathrm{kcal} / \mathrm{mol})$ & B3LYP $(\mathrm{kcal} / \mathrm{mol})$ \\
\hline $6-31 \mathrm{G}(\mathrm{d})$ & -250243.65435 & -250575.22533 \\
$6-31 \mathrm{G}(2 \mathrm{~d})$ & -250256.40862 & -250577.23986 \\
$6-31 \mathrm{G}(\mathrm{d}, \mathrm{p})$ & -250257.32467 & -250579.15187 \\
$6-31+\mathrm{G}(\mathrm{d})$ & -250244.76749 & -250576.39538 \\
$6-31 \mathrm{G}+(2 \mathrm{~d}, \mathrm{p})$ & -250270.24063 & -250582.29571 \\
$6-311 \mathrm{G}(\mathrm{d})$ & -250263.47234 & -250593.26484 \\
$6-311(3 \mathrm{df}, 2 \mathrm{p})$ & -250311.81844 & -250603.20855 \\
$6-311++G(\mathrm{~d})$ & -250264.31644 & -250593.7648 \\
$6-311++G(3 \mathrm{df}, 2 \mathrm{p})$ & -250312.13480 & -250603.58962 \\
\hline G3 (298.15K) & -250524.2818 \\
G3 (0K) & -250526.0689 \\
\hline
\end{tabular}

TOTAL MULLIKEN AND LOWDIN ATOMIC POPULATIONS

\begin{tabular}{lrrrr} 
ATOM & MULL.POP & \multicolumn{1}{c}{ CHARGE } & \multicolumn{1}{c}{ LOW.POP. } & \multicolumn{1}{c}{ CHARGE } \\
$1 \mathrm{H}$ & 0.930177 & 0.069823 & 0.931204 & 0.068796 \\
$2 \mathrm{~S}$ & 16.139647 & -0.139647 & 16.137593 & -0.137593 \\
$3 \mathrm{H}$ & 0.930177 & 0.069823 & 0.931204 & 0.068796
\end{tabular}

\section{${ }^{3}$ carbomethoxycarbene}

$\begin{array}{lrrrr}\text { MP2 } / 6-31 G(d, p) & & & \\ \mathrm{O} & 8.0 & -1.1380377594 & -0.1701499695 & -1.0215712642 \\ \mathrm{C} & 6.0 & -0.8666653671 & -0.0190309967 & 0.1615518003 \\ \mathrm{O} & 8.0 & 0.3875348640 & 0.0937383148 & 0.6534391713 \\ \mathrm{C} & 6.0 & -1.8687571330 & 0.0613052059 & 1.2141283236 \\ \mathrm{H} & 1.0 & -2.9385016061 & 0.0029079750 & 1.1101718382 \\ \mathrm{C} & 6.0 & 1.4134404843 & 0.0199492250 & -0.3545468162 \\ \mathrm{H} & 1.0 & 2.3470060928 & 0.1254966772 & 0.1858049925 \\ \mathrm{H} & 1.0 & 1.2928891472 & 0.8218389853 & -1.0786291782 \\ \mathrm{H} & 1.0 & 1.3710512770 & -0.9360554171 & -0.8703388674\end{array}$


$\operatorname{MP} 2 / 6-31 G(d, p)$ Energy

ZPE Correction

298.15K Temperature Correction

MP2 / 6-311G (3df, 2p) Energy
$-167092.4429 \mathrm{KCAL} / \mathrm{MOL}$

$41.5974 \mathrm{KCAL} / \mathrm{MOL}$

$45.64 \mathrm{KCAL} / \mathrm{MOL}$

$-167276.1669 \mathrm{KCAL} / \mathrm{MOL}$

TOTAL MULLIKEN AND LOWDIN ATOMIC POPULATIONS

\begin{tabular}{|c|c|c|c|c|}
\hline ATOM & MULL . POP . & CHARGE & LOW . POP. & CHARGE \\
\hline 10 & 8.443306 & -0.443306 & 8.247754 & -0.247754 \\
\hline $\mathrm{C}$ & 5.434905 & 0.565095 & 5.818626 & 0.181374 \\
\hline 0 & 8.485192 & -0.485192 & 8.200444 & -0.200444 \\
\hline $\mathrm{C}$ & 6.121907 & -0.121907 & 6.076128 & -0.076128 \\
\hline $\mathrm{H}$ & 0.836035 & 0.163965 & 0.866543 & 0.133457 \\
\hline $6 \mathrm{C}$ & 6.084238 & -0.084238 & 6.127551 & -0.127551 \\
\hline $\mathrm{H}$ & 0.865658 & 0.134342 & 0.885058 & 0.114942 \\
\hline $\mathrm{H}$ & 0.864366 & 0.135634 & 0.888363 & 0.111637 \\
\hline $\mathrm{H}$ & 0.864394 & 0.135606 & 0.889534 & 0.110466 \\
\hline
\end{tabular}

\section{${ }^{3}$ dicarbomethoxycarbene}

$\operatorname{MP} 2 / 6-31 G(d, p)$

\begin{tabular}{|c|c|c|c|c|}
\hline $\mathrm{C}$ & 6.0 & -.5023634738 & -.0006562954 & -.2142555325 \\
\hline $\mathrm{C}$ & 6.0 & -.4483548889 & -.1244418933 & 1.2411814800 \\
\hline $\mathrm{C}$ & 6.0 & .5849545679 & .1250182628 & -1.1829933020 \\
\hline O & 8.0 & .5390364723 & -.4859056981 & 1.8620682268 \\
\hline O & 8.0 & 1.7156863398 & .4895288911 & -.9005633909 \\
\hline O & 8.0 & -1.6417758000 & .1869784562 & 1.7907129184 \\
\hline O & 8.0 & .1561471936 & -.1886796545 & -2.4243542421 \\
\hline C & 6.0 & 1.1718366688 & -.0512994823 & -3.4386433969 \\
\hline $\mathrm{H}$ & 1.0 & 1.5268862006 & .9752727229 & -3.480929421 \\
\hline $\mathrm{H}$ & 1.0 & 2.0085990294 & -.7118051467 & -3.226606795 \\
\hline $\mathrm{H}$ & 1.0 & .6827022351 & -.3306807010 & -4.3643507 \\
\hline $\mathrm{C}$ & 6.0 & -1.6701750166 & .0511530815 & 3.2259887379 \\
\hline $\mathrm{H}$ & 1.0 & -.9381863810 & .7125678101 & 3.68215922 \\
\hline $\mathrm{H}$ & 1.0 & -2.6769793832 & .3301517709 & 3.5137781 \\
\hline $\mathrm{H}$ & 1.0 & -1.4542592801 & -.9749900035 & 3.512636707 \\
\hline \multirow{3}{*}{\multicolumn{3}{|c|}{$\begin{array}{l}\text { MP2/6-31G (d,p) Energy } \\
\text { ZPE Correction } \\
\text { 298.15K Temperature Correction }\end{array}$}} & 9698.7107 & $\mathrm{KCAL} / \mathrm{MO}$ \\
\hline & & & 0037 & $\mathrm{KCAL} / \mathrm{MOL}$ \\
\hline & & & & KCAL/MOL \\
\hline \multicolumn{3}{|c|}{ MP2/6-311G (3df,2p) Energy } & 131 & \\
\hline
\end{tabular}




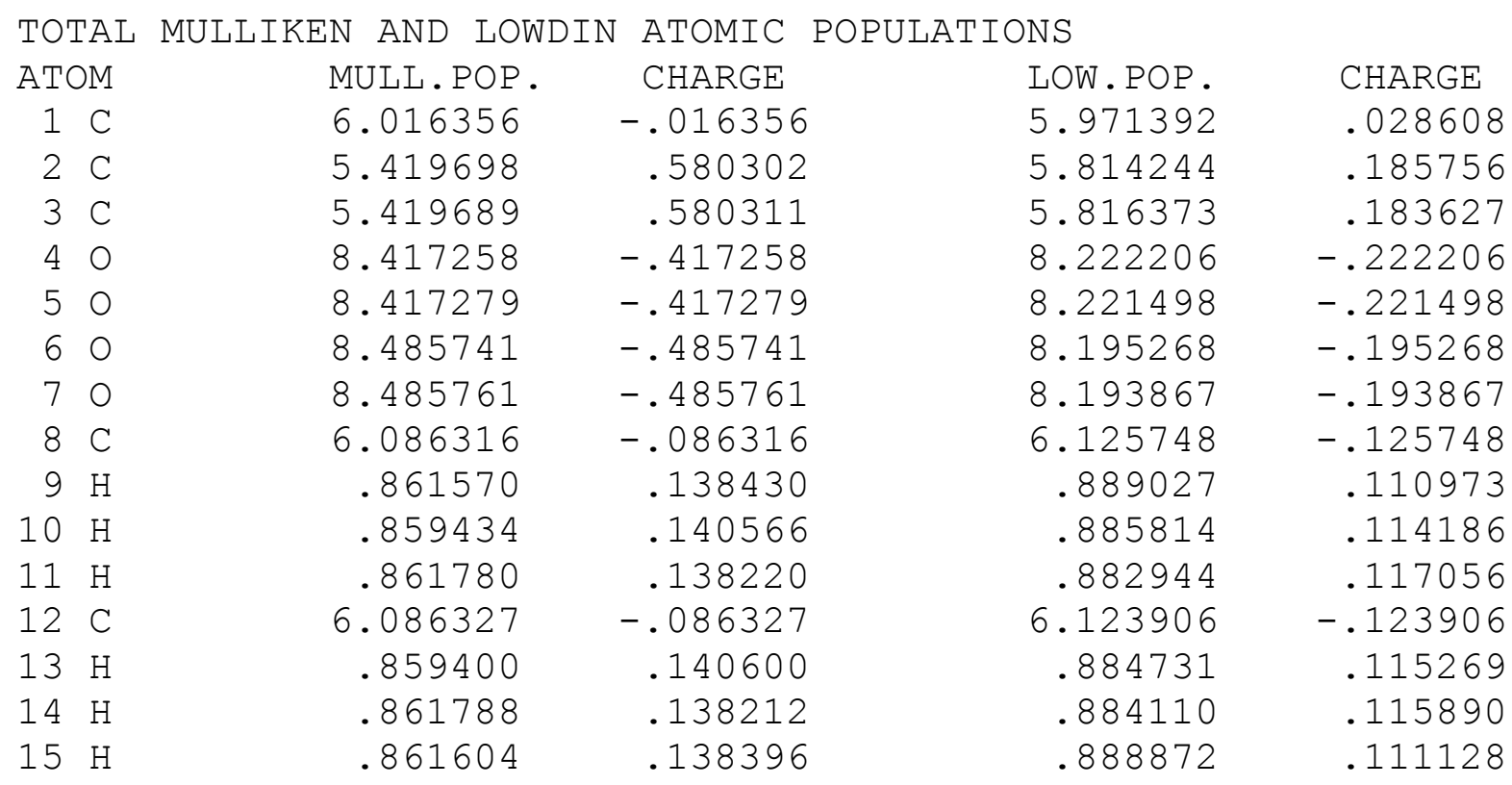

\section{dimethylsulfide}

$\operatorname{MP} 2 / 6-31 G(d, p)$

$\begin{array}{lrrrr}\mathrm{C} & 6.0 & -.9356015305 & -.6682076868 & -.7394741407 \\ \mathrm{~S} & 16.0 & -.8185911339 & .8455464993 & .2362777688 \\ \mathrm{C} & 6.0 & .9057452030 & .6986579416 & .7484506166 \\ \mathrm{H} & 1.0 & 1.5732196651 & .6864359744 & -.1114430910 \\ \mathrm{H} & 1.0 & 1.0663234024 & -.1983516082 & 1.3438444577 \\ \mathrm{H} & 1.0 & 1.1429734108 & 1.5668362514 & 1.3586847004 \\ \mathrm{H} & 1.0 & -.2350057657 & -.6559080958 & -1.5725990999 \\ \mathrm{H} & 1.0 & -1.9459031085 & -.7260693222 & -1.1373993668 \\ \mathrm{H} & 1.0 & -.7531606187 & -1.5489396887 & -.1263417921\end{array}$

MP2 / 6-31G (d,p) Energy

ZPE Correction

298.15K Temperature Correction
$-299428.53866 \mathrm{KCAL} / \mathrm{MOL}$

$49.41 \mathrm{KCAL} / \mathrm{MOL}$

$52.995 \mathrm{KCAL} / \mathrm{MOL}$ 


\begin{tabular}{lcc}
\hline Basis Set & MP2 $(\mathrm{kcal} / \mathrm{mol})$ & B3LYP $(\mathrm{kcal} / \mathrm{mol})$ \\
\hline $6-31 \mathrm{G}(\mathrm{d})$ & -299398.21971 & -299878.54633 \\
$6-31 \mathrm{G}(\mathrm{d})$ & -299420.36864 & -299878.29406 \\
$6-31 \mathrm{G}(\mathrm{d}, \mathrm{p})$ & -299428.53866 & -299883.91823 \\
$6-31+\mathrm{G}(\mathrm{d})$ & -299401.19796 & -299881.02809 \\
$6-31 \mathrm{G}+(2 \mathrm{~d}, \mathrm{p})$ & -299453.31291 & -299887.44316 \\
$6-311 \mathrm{G}(\mathrm{d})$ & -299440.87434 & -299907.76820 \\
$6-311(3 \mathrm{df}, 2 \mathrm{p})$ & -299531.18690 & -299921.17182 \\
$6-311++\mathrm{G}(\mathrm{d})$ & -299443.10333 & -299908.54632 \\
$6-311++\mathrm{G}(3 \mathrm{df}, 2 \mathrm{p})$ & -299531.91535 & -299921.67480 \\
\hline G3 (298.15K) & -299802.6465 \\
G3 (0K) & -299805.7476 \\
\hline
\end{tabular}

TOTAL MULLIKEN AND LOWDIN ATOMIC POPULATIONS

\begin{tabular}{llrrrr} 
ATOM & \multicolumn{1}{c}{ MULL.POP } & \multicolumn{1}{c}{ CHARGE } & \multicolumn{1}{c}{ LOW.POP. } & \multicolumn{1}{c}{ CHARGE } \\
1 & $\mathrm{C}$ & 6.464376 & -0.464376 & 6.406122 & -0.406122 \\
$2 \mathrm{~S}$ & 15.896877 & 0.103123 & 15.878172 & 0.121828 \\
$3 \mathrm{C}$ & 6.464376 & -0.464376 & 6.408067 & -0.408067 \\
4 & $\mathrm{H}$ & 0.866308 & 0.133692 & 0.889748 & 0.110252 \\
$5 \mathrm{H}$ & 0.866120 & 0.133880 & 0.890064 & 0.109936 \\
$6 \mathrm{H}$ & 0.854757 & 0.145243 & 0.873396 & 0.126604 \\
$7 \mathrm{H}$ & 0.866309 & 0.133691 & 0.889639 & 0.110361 \\
$8 \mathrm{H}$ & 0.854757 & 0.145243 & 0.875091 & 0.124909 \\
$9 \mathrm{H}$ & 0.866120 & 0.133880 & 0.889700 & 0.110300
\end{tabular}

\section{$\mathrm{CH}_{3} \mathrm{SH}$}

$\operatorname{MP} 2 / 6-31 G(d, p)$

$\begin{array}{rrrrr}\mathrm{C} & 6.0 & 0.0944885526 & 0.4764196287 & -0.0669216366 \\ \mathrm{H} & 1.0 & 1.1176693744 & 0.4889201579 & 0.2971684725 \\ \mathrm{~S} & 16.0 & -0.8321068124 & -0.9378791716 & 0.5906587973 \\ \mathrm{H} & 1.0 & -0.4080602493 & 1.3713421983 & 0.2893290366 \\ \mathrm{H} & 1.0 & -0.0679700276 & -1.8833906230 & 0.0493601256 \\ \mathrm{H} & 1.0 & 0.0891614033 & 0.4885774335 & -1.1529173754\end{array}$

MP2 / 6-31G (d,p) Energy

ZPE Correction

$-274841.7152 \mathrm{KCAL} / \mathrm{MOL}$

$30.12 \mathrm{KCAL} / \mathrm{MOL}$

298.15K Temperature Correction

$32.926 \mathrm{KCAL} / \mathrm{MOL}$ 


\begin{tabular}{lcc}
\hline Basis Set & MP2 $(\mathrm{kcal} / \mathrm{mol})$ & B3LYP $(\mathrm{kcal} / \mathrm{mol})$ \\
\hline $6-31 \mathrm{G}(\mathrm{d})$ & -274819.6223 & -275226.1094 \\
$6-31 \mathrm{G}(2 \mathrm{~d})$ & -274836.7898 & -275226.9354 \\
$6-31 \mathrm{G}(\mathrm{d}, \mathrm{p})$ & -274841.7152 & -275230.7364 \\
$6-31+\mathrm{G}(\mathrm{d})$ & -274821.5476 & -275227.9033 \\
$6-311 \mathrm{G}(\mathrm{d})$ & -274850.7988 & -275249.7059 \\
$6-311 \mathrm{G}(3 \mathrm{df}, 2 \mathrm{p})$ & -274919.9373 & -275261.2911 \\
$6-311++\mathrm{G}(\mathrm{d})$ & -274852.2258 & -275250.3488 \\
$6-311++\mathrm{G}(3 \mathrm{df}, 2 \mathrm{p})$ & -274920.4715 & -275261.7396 \\
\hline G3 (298.15K) & -275161.8681 \\
G3 (0K) & -275164.1522 \\
\hline
\end{tabular}

TOTAL MULLIKEN AND LOWDIN ATOMIC POPULATIONS

\begin{tabular}{|c|c|c|c|c|}
\hline ATOM & MULL . POP . & CHARGE & LOW . POP . & CHARGE \\
\hline $1 \mathrm{C}$ & 6.467140 & -.467140 & 6.398952 & -.398952 \\
\hline $2 \mathrm{H}$ & .856620 & .143380 & .886547 & .113453 \\
\hline $3 \mathrm{~s}$ & 16.021335 & -.021335 & 16.009563 & -.009563 \\
\hline $\mathrm{H}$ & .851733 & .148267 & .873969 & .126031 \\
\hline $5 \mathrm{H}$ & .946557 & .053443 & 988 & .057012 \\
\hline $6 \mathrm{H}$ & .856615 & .143385 & .887981 & .112019 \\
\hline
\end{tabular}

ethenethiol

$\operatorname{MP} 2 / 6-31 G(d, p)$

$\begin{array}{rrrrr}\mathrm{H} & 1.0 & -1.7151159828 & 0.2000932598 & -1.0404537998 \\ \mathrm{~S} & 16.0 & -1.8408670513 & 0.1113105377 & 0.2829151987 \\ \mathrm{C} & 6.0 & -0.1197259641 & -0.0230879710 & 0.6295453206 \\ \mathrm{C} & 6.0 & 0.8925529634 & -0.0229748723 & -0.2457525270 \\ \mathrm{H} & 1.0 & 0.0724046078 & -0.1127358588 & 1.6908421195 \\ \mathrm{H} & 1.0 & 1.9099463949 & -0.1127046837 & 0.1039414324 \\ \mathrm{H} & 1.0 & 0.7352248682 & 0.0651871553 & -1.3117131105\end{array}$

MP2 / 6-31G (d,p) Energy

ZPE Correction

$-298663.89536 \mathrm{KCAL} / \mathrm{MOL}$

$33.08 \mathrm{KCAL} / \mathrm{MOL}$

298.15K Temperature Correction

$36.225 \mathrm{KCAL} / \mathrm{MOL}$

\begin{tabular}{lcc}
\hline Basis Set & MP2 $(\mathrm{kcal} / \mathrm{mol})$ & B3LYP $(\mathrm{kcal} / \mathrm{mol})$ \\
\hline $6-31 \mathrm{G}(\mathrm{d})$ & -298641.76791 & -299106.86099 \\
$6-31 \mathrm{G}(2 \mathrm{~d})$ & -298664.32031 & -299108.28517 \\
$6-31 \mathrm{G}(\mathrm{d}, \mathrm{p})$ & -298663.89536 & -299111.87120 \\
$6-31+\mathrm{G}(\mathrm{d})$ & -298646.48031 & -299110.64823 \\
$6-311 \mathrm{G}(\mathrm{d})$ & -298684.33162 & -299136.58707 \\
$6-311 \mathrm{G}(3 \mathrm{df}, 2 \mathrm{p})$ & -298764.73834 & -299151.36067 \\
$6-311++\mathrm{G}(\mathrm{d})$ & -298682.92185 & -299137.90015 \\
$6-311++\mathrm{G}(3 \mathrm{df}, 2 \mathrm{p})$ & -298765.99031 & -299152.33862 \\
\hline G3 (298.15K) & -299044.792 \\
G3 (0K) & -299047.4169 \\
\hline
\end{tabular}




\begin{tabular}{lcrrr}
\multicolumn{1}{l}{ TOTAL MULLIKEN AND LOWDIN } & ATOMIC & POPULATIONS \\
ATOM & MULL.POP. & CHARGE & LOW. POP. & CHARGE \\
$1 \mathrm{H}$ & .944531 & .055469 & .941100 & .058900 \\
$2 \mathrm{~S}$ & 15.958152 & .041848 & 15.935407 & .064593 \\
$3 \mathrm{C}$ & 6.230910 & -.230910 & 6.218118 & -.218118 \\
$4 \mathrm{C}$ & 6.247625 & -.247625 & 6.256502 & -.256502 \\
$5 \mathrm{H}$ & .852896 & .147104 & .875161 & .124839 \\
$6 \mathrm{H}$ & .876206 & .123794 & .881486 & .118514 \\
$7 \mathrm{H}$ & .889679 & .110321 & .892225 & .107775
\end{tabular}

\section{thiirane}

MP2 / 6-31g (d,p)

$\begin{array}{lrrrr}\mathrm{H} & 1.0 & -1.2466717881 & .9115354684 & .3591001536 \\ \mathrm{C} & 6.0 & -.7402086504 & -.0000000004 & .0732107641 \\ \mathrm{C} & 6.0 & .7402086461 & -.0000000002 & .0732107603 \\ \mathrm{H} & 1.0 & -1.2466717885 & -.9115354687 & .3591001533 \\ \mathrm{H} & 1.0 & 1.2466717904 & .9115354723 & .3591001538 \\ \mathrm{H} & 1.0 & 1.2466717889 & -.9115354713 & .3591001538 \\ \mathrm{~S} & 16.0 & .0000000016 & -.0000000001 & -1.5828221399 \\ & & -298670.3413 \mathrm{KCAL} / \mathrm{MOL} \\ \mathrm{MP} 2 / 6-31 \mathrm{G}(\mathrm{d}, \mathrm{p}) & \text { Energy } & 35.87 & \mathrm{KCAL} / \mathrm{MOL} \\ \mathrm{ZPE} \text { Correction } & & 38.528 & \mathrm{KCAL} / \mathrm{MOL}\end{array}$

\begin{tabular}{lcc}
\hline Basis Set & MP2 $(\mathrm{kcal} / \mathrm{mol})$ & B3LYP $(\mathrm{kcal} / \mathrm{mol})$ \\
\hline $6-31 \mathrm{G}(\mathrm{d})$ & -298650.3138 & -299110.8023 \\
$6-31 \mathrm{G}(2 \mathrm{~d})$ & -298671.5823 & -299110.9153 \\
$6-31 \mathrm{G}(\mathrm{d}, \mathrm{p})$ & -298670.3413 & -299114.4541 \\
$6-31+\mathrm{G}(\mathrm{d})$ & -298653.6779 & -299113.274 \\
$6-31+\mathrm{G}(2 \mathrm{~d}, \mathrm{p})$ & -298694.1158 & -299118.0182 \\
$6-311 \mathrm{G}(\mathrm{d})$ & -298691.5966 & -299139.3633 \\
$6-311 \mathrm{G}(3 \mathrm{df}, 2 \mathrm{p})$ & -298770.8459 & -299151.578 \\
$6-311++\mathrm{G}(\mathrm{d})$ & -298693.5236 & -299140.1352 \\
$6-311++\mathrm{G}(3 \mathrm{df}, 2 \mathrm{p})$ & -298771.6278 & -299152.1221 \\
\hline $\mathrm{G} 3(298.15 \mathrm{~K})$ & -299044.9426 \\
$G 3(0 \mathrm{~K})$ & -299047.078 \\
\hline
\end{tabular}


TOTAL MULLIKEN AND LOWDIN ATOMIC POPULATIONS

\begin{tabular}{llrrrr} 
ATOM & MULL.POP. & \multicolumn{1}{c}{ CHARGE } & \multicolumn{1}{c}{ LOW.POP. } & \multicolumn{1}{c}{ CHARGE } \\
$1 \mathrm{H}$ & 0.843151 & 0.156849 & 0.876679 & 0.123321 \\
$2 \mathrm{C}$ & 6.316714 & -0.316714 & 6.265985 & -0.265985 \\
$3 \mathrm{C}$ & 6.316714 & -0.316714 & 6.265985 & -0.265985 \\
$4 \mathrm{H}$ & 0.843151 & 0.156849 & 0.876679 & 0.123321 \\
$5 \mathrm{H}$ & 0.843151 & 0.156849 & 0.876679 & 0.123321 \\
$6 \mathrm{H}$ & 0.843151 & 0.156849 & 0.876679 & 0.123321 \\
$7 \mathrm{~S}$ & 15.993969 & 0.006031 & 15.961314 & 0.038686
\end{tabular}

\section{methyl vinyl sulfide}

$\operatorname{MP} 2 / 6-31 G(d, p)$

$\begin{array}{lrrrr}\mathrm{C} & 6.0 & -.2510613838 & -1.3513930757 & -.7406270654 \\ \mathrm{~S} & 16.0 & -1.5221887952 & -.2826840183 & -.0431148871 \\ \mathrm{C} & 6.0 & -.5426244717 & 1.0150413208 & .6100440214 \\ \mathrm{H} & 1.0 & .4240121313 & -1.7130982228 & .0319879677 \\ \mathrm{H} & 1.0 & .3166146053 & -.8316541694 & -1.5094483548 \\ \mathrm{H} & 1.0 & -.7616947340 & -2.1991607342 & -1.1899517996 \\ \mathrm{C} & 6.0 & .7917601161 & 1.1473203768 & .5882011422 \\ \mathrm{H} & 1.0 & -1.1538880824 & 1.7774471600 & 1.0775379746 \\ \mathrm{H} & 1.0 & 1.4509763914 & .4208412359 & .1389781919 \\ \mathrm{H} & 1.0 & 1.2480922641 & 2.0173407620 & 1.036393380\end{array}$

MP2 / 6-31G (d,p) Energy

ZPE Correction

$-323251.8287 \mathrm{KCAL} / \mathrm{MOL}$ $52.51 \mathrm{KCAL} / \mathrm{MOL}$ 298.15K Temperature Correction

$56.414 \mathrm{KCAL} / \mathrm{MOL}$

\begin{tabular}{lcc}
\hline Basis Set & MP2 (kcal/mol) & B3LYP $(\mathrm{kcal} / \mathrm{mol})$ \\
\hline $6-31 G(\mathrm{~d})$ & -323221.3369 & -323760.1308 \\
$6-31 \mathrm{G}(2 \mathrm{~d})$ & -323249.212 & -323760.5558 \\
$6-31 \mathrm{G}(\mathrm{d}, \mathrm{p})$ & -323251.8287 & -323765.9305 \\
$6-31+\mathrm{G}(\mathrm{d})$ & -323227.0857 & -323764.6355 \\
$6-31+\mathrm{G}(2 \mathrm{~d}, \mathrm{p})$ & -323284.207 & -323772.1336 \\
$6-311 \mathrm{G}(\mathrm{d})$ & -323272.9771 & -323795.4542 \\
$6-311 \mathrm{G}(3 \mathrm{df}, 2 \mathrm{p})$ & -323377.7002 & -323812.2837 \\
$6-311++\mathrm{G}(\mathrm{d})$ & -323276.1397 & -323796.9318 \\
$6-311++\mathrm{G}(3 \mathrm{df}, 2 \mathrm{p})$ & -323379.2241 & -323813.3946 \\
\hline G3 (298.15K) & \multicolumn{2}{c}{-323687.0319} \\
G3 $(0 \mathrm{~K})$ & \multicolumn{2}{c}{-323690.4643} \\
\hline
\end{tabular}




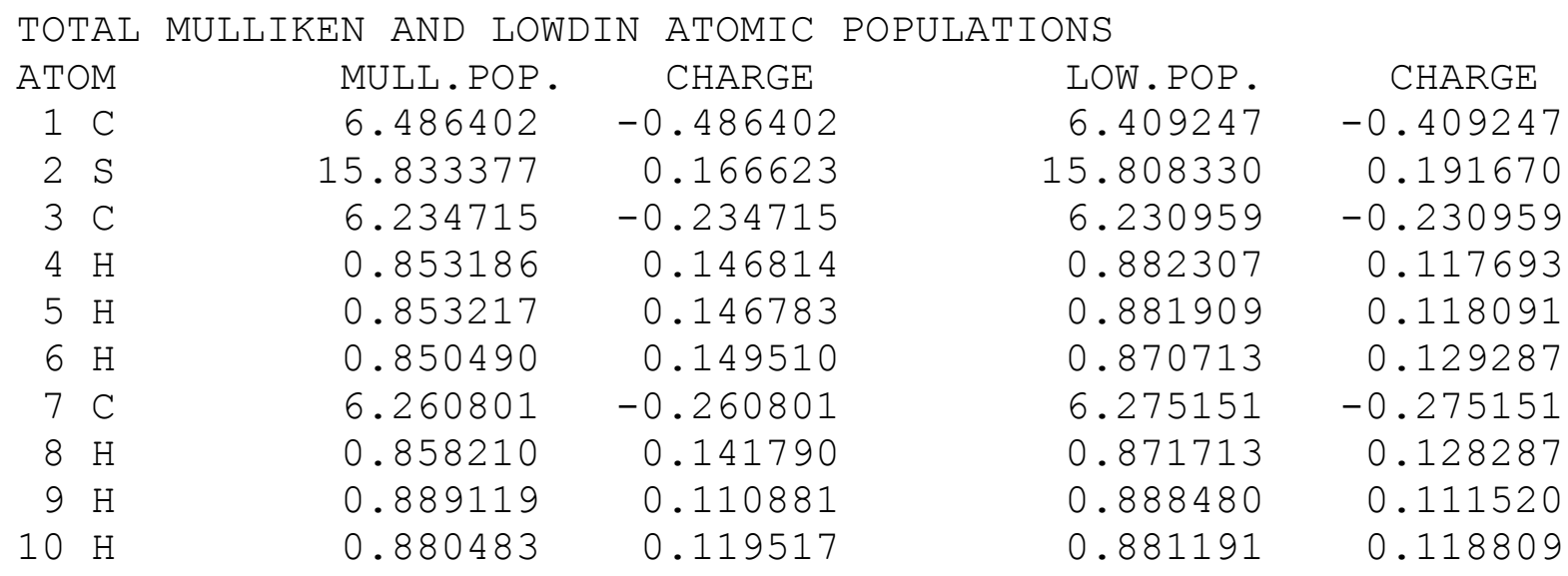

\section{diethyl sulfide}

$\operatorname{MP} 2 / 6-31 G(d, p)$

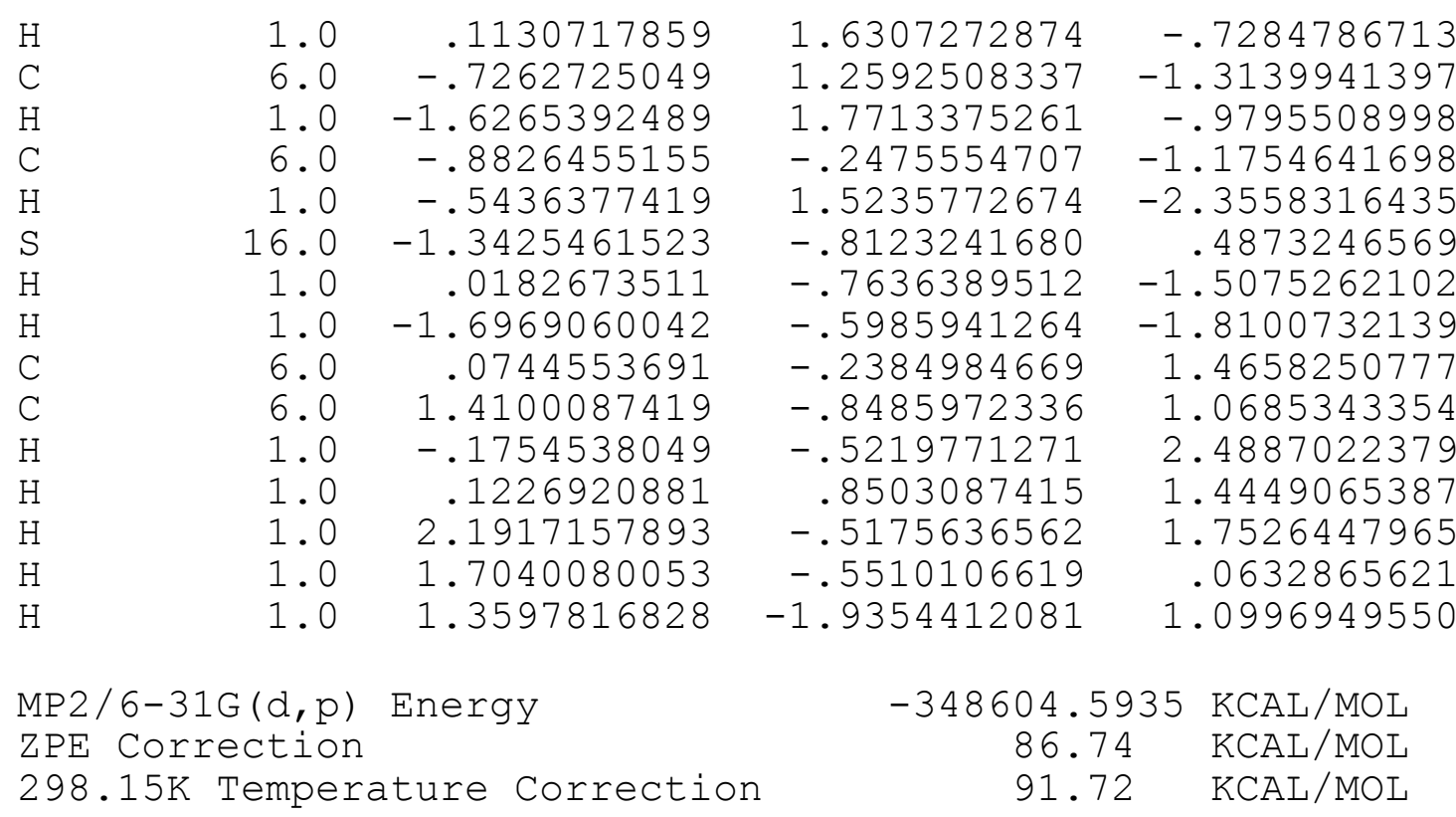




\begin{tabular}{lcc}
\hline Basis Set & MP2 (kcal/mol) & B3LYP $(\mathrm{kcal} / \mathrm{mol})$ \\
\hline $6-31 G(\mathrm{~d})$ & -348553.4483 & -349181.67 \\
$6-31 G(2 \mathrm{~d})$ & -348586.6531 & -349180.8646 \\
$6-31 \mathrm{G}(\mathrm{d}, \mathrm{p})$ & -348604.5935 & -349190.5021 \\
$6-31+\mathrm{G}(\mathrm{d})$ & -348558.7049 & -349185.4464 \\
$6-31+\mathrm{G}(2 \mathrm{~d}, \mathrm{p})$ & -348641.0076 & -349195.2273 \\
$6-311 \mathrm{G}(\mathrm{d})$ & -348617.0452 & -349222.8724 \\
$6-311 G(3 \mathrm{df}, 2 \mathrm{p})$ & -348755.9586 & -349241.5496 \\
$6-311++\mathrm{G}(\mathrm{d})$ & -348620.0984 & -349222.8724 \\
$6-311++\mathrm{G}(3 \mathrm{df}, 2 \mathrm{p})$ & -348757.0943 & -349242.1869 \\
\hline G3 (298.15K) & -349088.1980 \\
G3 (0K) & -349092.9138 \\
\hline
\end{tabular}

TOTAL MULLIKEN AND LOWDIN ATOMIC POPULATIONS

\begin{tabular}{|c|c|c|c|c|}
\hline ATOM & MULL.POP & CHARGE & LOW. POP. & CHARGE \\
\hline $1 \mathrm{H}$ & .886542 & .113458 & .895836 & .104164 \\
\hline 20 & 6.331503 & -.331503 & 6.310208 & -.310208 \\
\hline $3 \mathrm{H}$ & .869929 & .130071 & .887542 & .112458 \\
\hline 4 & 6.353471 & -.353471 & 6.293367 & -.293367 \\
\hline 5 & .883844 & .116156 & .894292 & .105708 \\
\hline 6 & 15.903643 & .096357 & 15.890693 & .109307 \\
\hline $7 \mathrm{H}$ & .865352 & .134648 & .894295 & .105705 \\
\hline 8 & .857480 & .142520 & .879184 & .120816 \\
\hline 9 & 6.353679 & -.353679 & 6.287542 & -.287542 \\
\hline 10 & 6.331028 & -.331028 & 6.307646 & -.307646 \\
\hline $11 \mathrm{H}$ & .857508 & .142492 & .881477 & .118523 \\
\hline $12 \mathrm{H}$ & .865308 & .134692 & .897667 & .10233 \\
\hline $13 \mathrm{H}$ & .884018 & .115982 & .891482 & .108518 \\
\hline 14 & .886631 & .113369 & .897876 & .102 \\
\hline $15 \mathrm{H}$ & .870065 & .129935 & .890893 & .109 \\
\hline
\end{tabular}




\section{allyl ethyl sulfide}

$\operatorname{MP} 2 / 6-31 G(d, p)$

$\begin{array}{rrrrr}\mathrm{H} & 1.0 & -0.4009802946 & 1.4272704198 & -1.7098930780 \\ \mathrm{C} & 6.0 & -1.2310604290 & 0.7333889416 & -1.6775947200 \\ \mathrm{C} & 6.0 & -1.7197177852 & 0.2871633598 & -0.5133568646 \\ \mathrm{H} & 1.0 & -1.6567530958 & 0.4300321168 & -2.6233056408 \\ \mathrm{C} & 6.0 & -1.1569062438 & 0.6609509795 & 0.8175579115 \\ \mathrm{H} & 1.0 & -2.5471097527 & -0.4140825197 & -0.5062956176 \\ \mathrm{~S} & 16.0 & -0.4124886089 & -0.7621337711 & 1.6828161057 \\ \mathrm{H} & 1.0 & -1.9436344650 & 1.0061427109 & 1.4902670755 \\ \mathrm{H} & 1.0 & -0.4315242691 & 1.4690715871 & 0.7186752356 \\ \mathrm{C} & 6.0 & 0.8827891563 & -1.2148849957 & 0.4962527809 \\ \mathrm{C} & 6.0 & 2.0153943118 & -0.2057237887 & 0.3863388573 \\ \mathrm{H} & 1.0 & 0.4248318923 & -1.3895294007 & -0.4774059968 \\ \mathrm{H} & 1.0 & 1.2621010488 & -2.1723610355 & 0.8542315636 \\ \mathrm{H} & 1.0 & 2.7788290153 & -0.5713743412 & -0.3010449488 \\ \mathrm{H} & 1.0 & 2.4763644251 & -0.0340947958 & 1.3570473521 \\ \mathrm{H} & 1.0 & 1.6598646696 & 0.7501633138 & 0.0057098796\end{array}$

MP2 / 6-31G (d,p) Energy

ZPE Correction

$-372427.0882 \mathrm{KCAL} / \mathrm{MOL}$

$89.40 \mathrm{KCAL} / \mathrm{MOL}$

298.15K Temperature Correction

$94.96 \mathrm{KCAL} / \mathrm{MOL}$

\begin{tabular}{lcc}
\hline Basis Set & MP2 $(\mathrm{kcal} / \mathrm{mol})$ & B3LYP $(\mathrm{kcal} / \mathrm{mol})$ \\
\hline $6-31 \mathrm{G}(\mathrm{d})$ & -372376.1199 & -373062.4294 \\
$6-31 \mathrm{G}(2 \mathrm{~d})$ & -372415.2141 & -373062.14 \\
$6-31 \mathrm{G}(\mathrm{d}, \mathrm{p})$ & -372427.0882 & -373071.6508 \\
$6-31+\mathrm{G}(\mathrm{d})$ & -372384.0753 & -373067.8323 \\
$6-31+\mathrm{G}(2 \mathrm{~d}, \mathrm{p})$ & -372471.1529 & -373078.6424 \\
$6-311 \mathrm{G}(\mathrm{d})$ & -372448.5966 & -373108.7626 \\
$6-311 \mathrm{G}(3 \mathrm{df}, 2 \mathrm{p})$ & -372601.6182 & -373131.2436 \\
$6-311++\mathrm{G}(\mathrm{d})$ & -372452.8722 & -373110.2244 \\
$6-311++\mathrm{G}(3 \mathrm{df}, 2 \mathrm{p})$ & -372603.3496 & -373132.3588 \\
\hline $\mathrm{G} 3(298.15 \mathrm{~K})$ & -372971.3560 & \\
G3 (0K) & -372976.5217 & \\
\hline
\end{tabular}




\begin{tabular}{|c|c|c|c|c|}
\hline ATOI & MULL . POP . & CHARGE & LOW. POP. & CHARGE \\
\hline 11 & 0.891897 & 0.108103 & 0.892059 & 0.107941 \\
\hline 2 & 6.252230 & -0.252230 & 6.239117 & -0.239117 \\
\hline 3 & 6.051998 & -0.051998 & 6.107903 & -0.107903 \\
\hline 4 & 0.882261 & 0.117739 & 0.885845 & 0.114155 \\
\hline 5 & 6.393801 & -0.393801 & 6.289389 & -0.289389 \\
\hline 6 & 0.875588 & 0.124412 & 0.886342 & 0.113658 \\
\hline 7 & 15.898221 & 0.101779 & 15.884813 & 0.115187 \\
\hline 8 & 0.847398 & 0.152602 & 0.872727 & 0.127273 \\
\hline 9 & 0.860521 & 0.139479 & 0.889510 & 0.110490 \\
\hline 10 & 6.355931 & -0.355931 & 6.290170 & -0.290170 \\
\hline 11 & 6.325972 & -0.325972 & 6.310042 & -0.310042 \\
\hline 12 & 0.857566 & 0.142434 & 0.889960 & 0.110040 \\
\hline 13 & 0.858913 & 0.141087 & 0.881731 & 0.118269 \\
\hline 14 & 0.885037 & 0.114963 & 0.892044 & 0.107956 \\
\hline $15 \mathrm{H}$ & 0.872298 & 0.127702 & 0.889875 & 0.110125 \\
\hline $16 \mathrm{H}$ & 0.890368 & 0.109632 & 0.898474 & 0.101526 \\
\hline
\end{tabular}

\section{diphenyl sulfide}

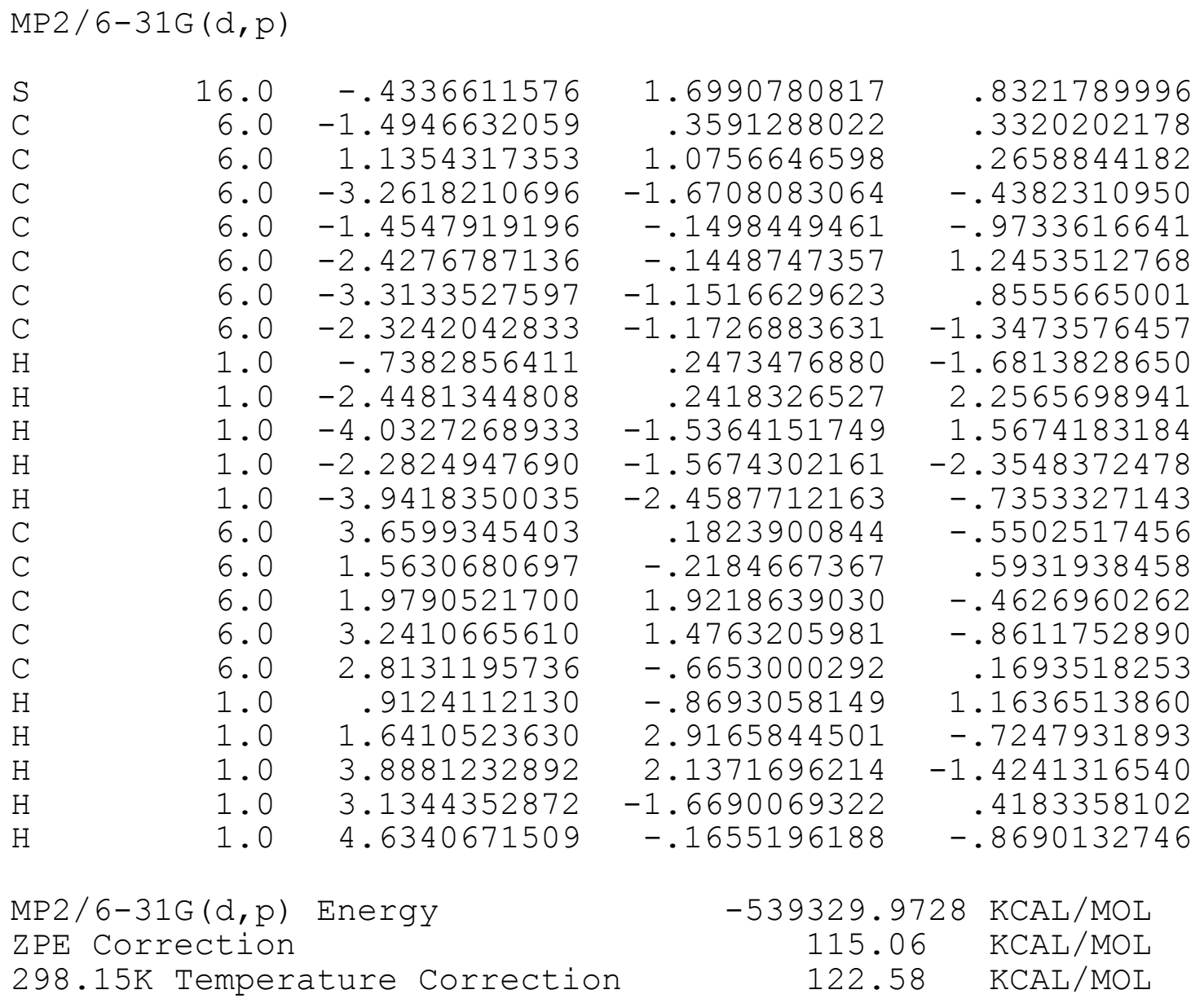




\begin{tabular}{lcc}
\hline Basis Set & MP2 $(\mathrm{kcal} / \mathrm{mol})$ & B3LYP $(\mathrm{kcal} / \mathrm{mol})$ \\
\hline $6-31 \mathrm{G}(\mathrm{d})$ & -539280.1292 & -540361.6415 \\
$6-31 \mathrm{G}(2 \mathrm{~d})$ & -539357.1008 & -540365.0398 \\
$6-31 \mathrm{G}(\mathrm{d}, \mathrm{p})$ & -539329.9728 & -540371.6461 \\
$6-31+\mathrm{G}(\mathrm{d})$ & -539300.4046 & -540373.0855 \\
$6-31+\mathrm{G}(2 \mathrm{~d}, \mathrm{p})$ & -539420.8938 & -540388.2683 \\
$6-311 \mathrm{G}(\mathrm{d})$ & -539408.4941 & -540442.262 \\
$6-311 \mathrm{G}(3 \mathrm{df}, 2 \mathrm{p})$ & -539660.9393 & -540481.9237 \\
$6-311++\mathrm{G}(\mathrm{d})$ & -539418.2606 & -540445.6606 \\
$6-311++\mathrm{G}(3 \mathrm{df}, 2 \mathrm{p})$ & -539664.7363 & -540484.1543 \\
\hline
\end{tabular}

TOTAL MULLIKEN AND LOWDIN ATOMIC POPULATIONS

\begin{tabular}{|c|c|c|c|c|c|}
\hline ATOI & & MULL . POP . & CHARGE & LOW . POP. & CHARGE \\
\hline 1 & S & 15.828917 & .171083 & 15.800284 & .199716 \\
\hline 2 & C & 6.173905 & -.173905 & 6.131341 & -.131341 \\
\hline 3 & C & 6.173943 & -.173943 & 6.131711 & -.131711 \\
\hline 4 & C & 6.123389 & -.123389 & 6.113363 & -.113363 \\
\hline 5 & $\mathrm{C}$ & 6.095005 & -.095005 & 6.112287 & -.112287 \\
\hline 6 & C & 6.115726 & -.115726 & 6.119181 & -.119181 \\
\hline 7 & C & 6.112760 & -.112760 & 6.108049 & -.108049 \\
\hline 8 & $\mathrm{C}$ & 6.113293 & -.113293 & 6.104728 & -.104728 \\
\hline 9 & $\mathrm{H}$ & .863607 & .136393 & .877052 & .122948 \\
\hline 10 & $\mathrm{H}$ & .864294 & .135706 & .880641 & .119359 \\
\hline 11 & $\mathrm{H}$ & .874054 & .125946 & .882936 & .117064 \\
\hline $12 \mathrm{I}$ & $\mathrm{H}$ & .873863 & .126137 & .885536 & .114464 \\
\hline 131 & $\mathrm{H}$ & .875663 & .124337 & .884608 & .115392 \\
\hline 14 & C & 6.123388 & -.123388 & 6.112192 & -.112192 \\
\hline 15 & C & 6.094991 & -.094991 & 6.112578 & -.112578 \\
\hline 16 & C & 6.115700 & -.115700 & 6.119447 & -.119447 \\
\hline 17 & C & 6.112767 & -.112767 & 6.108431 & -.108431 \\
\hline 18 & C & 6.113289 & -.113289 & 6.104647 & -.104647 \\
\hline 19 & $\mathrm{H}$ & .863600 & .136400 & .876672 & .123328 \\
\hline 20 & $\mathrm{H}$ & .864284 & .135716 & .880365 & .119635 \\
\hline 21 & $\mathrm{H}$ & .874049 & .125951 & .882507 & .117493 \\
\hline 22 & $\mathrm{H}$ & .873856 & .126144 & .885441 & .114559 \\
\hline 23 & $\mathrm{H}$ & .875658 & .124342 & .886005 & .113995 \\
\hline
\end{tabular}




\section{thiophene}

$\operatorname{MP} 2 / 6-31 G(d, p)$

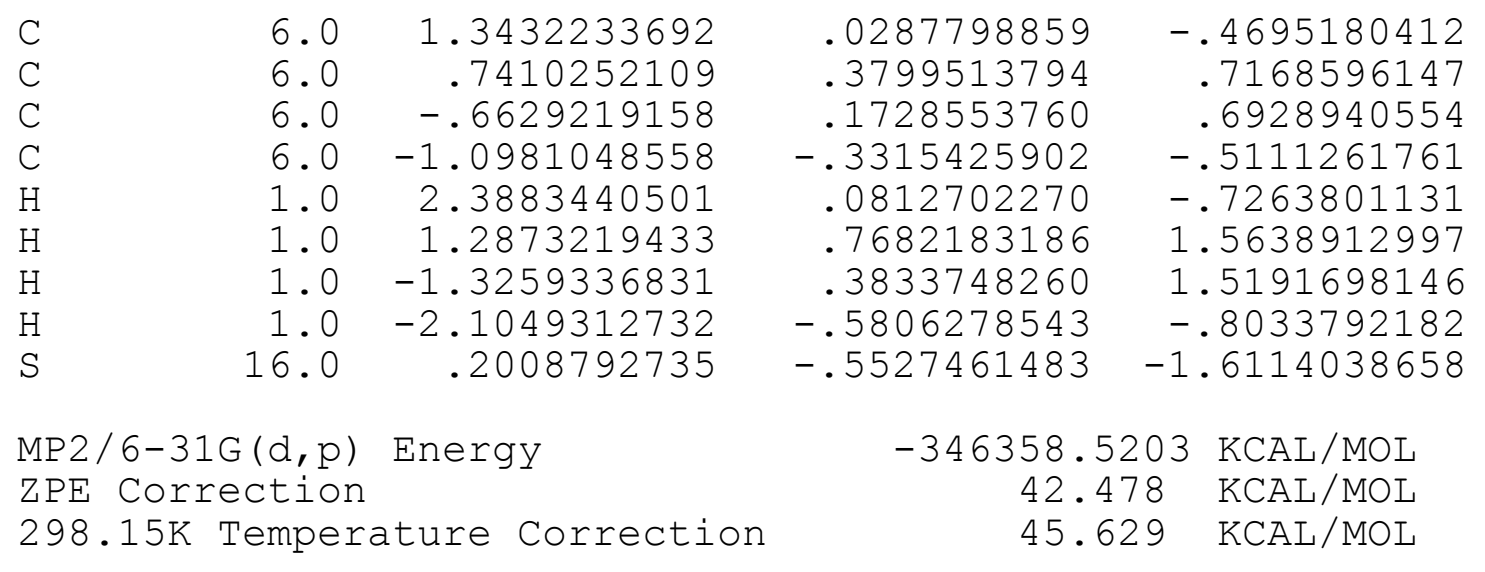

\begin{tabular}{lcc}
\hline Basis Set & MP2 $(\mathrm{kcal} / \mathrm{mol})$ & B3LYP $(\mathrm{kcal} / \mathrm{mol})$ \\
\hline $6-31 \mathrm{G}(\mathrm{d})$ & -346338.4039 & -346911.1695 \\
$6-31 \mathrm{G}(2 \mathrm{~d})$ & -346371.3186 & -346913.2678 \\
$6-31 \mathrm{G}(\mathrm{d}, \mathrm{p})$ & -346358.5203 & -346915.3726 \\
$6-31+\mathrm{G}(\mathrm{d})$ & -346346.0829 & -346916.7319 \\
$6-31+\mathrm{G}(2 \mathrm{~d}, \mathrm{p})$ & -346397.7064 & -346924.1867 \\
$6-311 \mathrm{G}(\mathrm{d})$ & -346396.6926 & -346950.8663 \\
$6-311 \mathrm{G}(3 \mathrm{df}, 2 \mathrm{p})$ & -346505.6794 & -346970.1119 \\
$6-311++\mathrm{G}(\mathrm{d})$ & -346400.5672 & -346952.8251 \\
$6-311++\mathrm{G}(3 \mathrm{df}, 2 \mathrm{p})$ & -346507.8208 & -346971.3842 \\
\hline $\mathrm{G} 3(298.15 \mathrm{~K})$ & -346850.0983 \\
$G 3(0 \mathrm{~K})$ & -346852.6999 \\
\hline
\end{tabular}

TOTAL MULLIKEN AND LOWDIN ATOMIC POPULATIONS

$\begin{array}{lrrrr}\text { ATOM } & \text { MULL.POP } & \text { CHARGE } & \text { LOW.POP. } & \text { CHARGE } \\ 1 \mathrm{C} & 6.332026 & -.332026 & 6.286543 & -.286543 \\ 2 \mathrm{C} & 6.095078 & -.095078 & 6.143178 & -.143178 \\ 3 \mathrm{C} & 6.095073 & -.095073 & 6.143205 & -.143205 \\ 4 \mathrm{C} & 6.332036 & -.332036 & 6.287697 & -.287697 \\ 5 \mathrm{H} & .846795 & .153205 & .869547 & .130453 \\ 6 \mathrm{H} & .871802 & .128198 & .878906 & .121094 \\ 7 \mathrm{H} & .871803 & .128197 & .878924 & .121076 \\ 8 \mathrm{H} & .846791 & .153209 & .868725 & .131275 \\ 9 \mathrm{~S} & 15.708598 & .291402 & 15.643277 & .356723\end{array}$




\section{benzothiophene}

MP 2 / 6-31G (d, p )

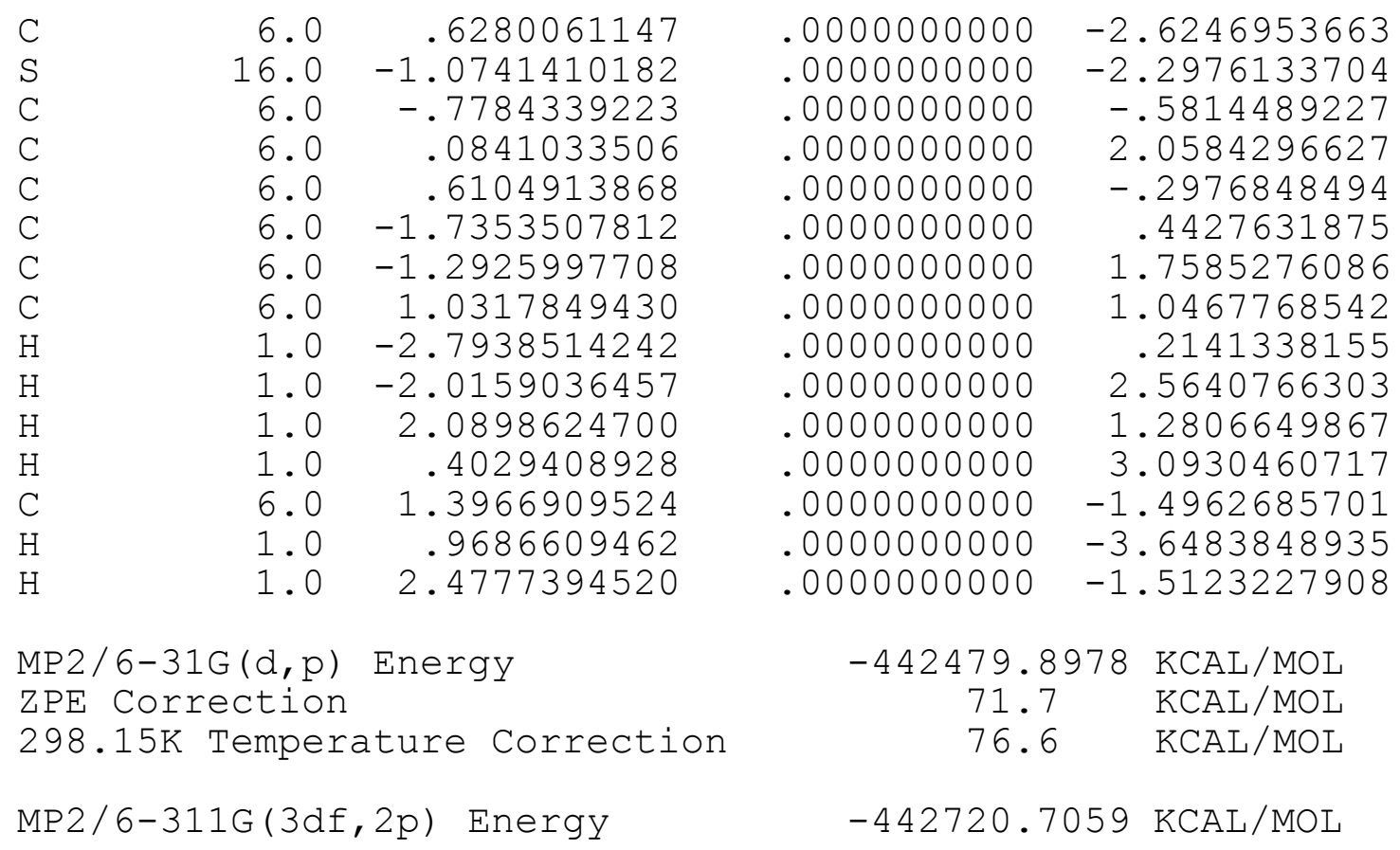

\begin{tabular}{|c|c|c|c|c|}
\hline АTOM & MULL . POP . & CHARGE & LOW. POP. & CHARGE \\
\hline $1 \mathrm{C}$ & 6.328220 & -0.328220 & 6.256950 & -0.256950 \\
\hline $2 \mathrm{~s}$ & 15.727380 & 0.272620 & 15.685029 & 0.314971 \\
\hline $\mathrm{C}$ & 6.206994 & -0.206994 & 6.150020 & -0.150020 \\
\hline $\mathrm{C}$ & 6.132718 & -0.132718 & 6.115236 & -0.115236 \\
\hline $\mathrm{C}$ & 5.960425 & 0.039575 & 6.024954 & -0.024954 \\
\hline C & 6.113145 & -0.113145 & 6.112655 & -0.112655 \\
\hline $\mathrm{C}$ & 6.117976 & -0.117976 & 6.115664 & -0.115664 \\
\hline $\mathrm{C}$ & 6.113014 & -0.113014 & 6.107925 & -0.107925 \\
\hline $\mathrm{H}$ & 0.869054 & 0.130946 & 0.883697 & 0.116303 \\
\hline $10 \mathrm{H}$ & 0.877595 & 0.122405 & 0.884206 & 0.115794 \\
\hline $11 \mathrm{H}$ & 0.876423 & 0.123577 & 0.887534 & 0.112466 \\
\hline $12 \mathrm{H}$ & 0.878278 & 0.121722 & 0.887740 & 0.112260 \\
\hline $13 \mathrm{C}$ & 6.086715 & -0.086715 & 6.138721 & -0.138721 \\
\hline $14 \mathrm{H}$ & 0.845209 & 0.154791 & 0.868747 & 0.131253 \\
\hline 15 & 0.866855 & 0.133145 & 0.880921 & 0.119079 \\
\hline
\end{tabular}




\section{dibenzothiophene}

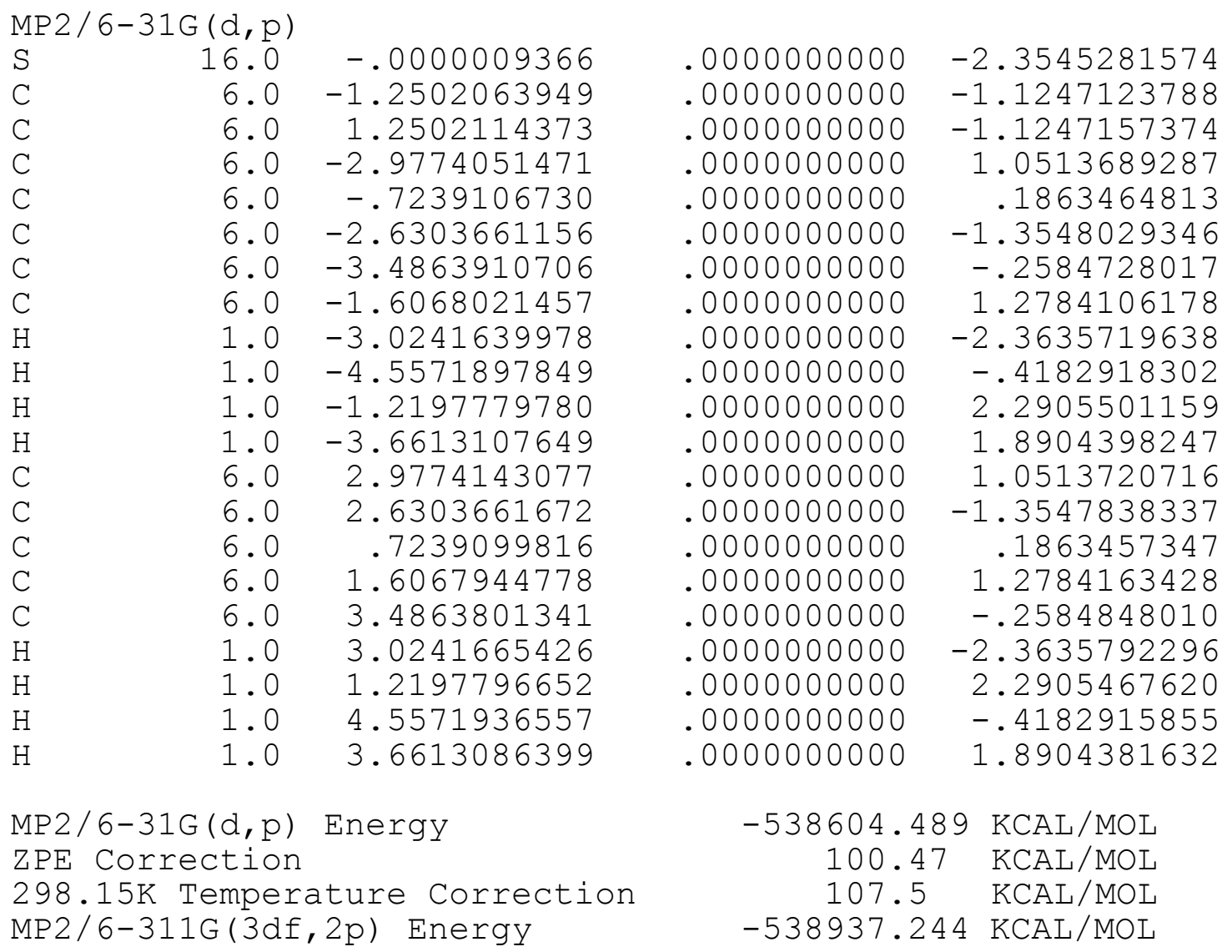




\begin{tabular}{rrrrrr}
\multicolumn{1}{l}{ TOTAL MULLIKEN AND LOWDIN ATOMIC } & POPULATIONS \\
ATOM & MULL.POP & \multicolumn{1}{c}{ CHARGE } & \multicolumn{1}{l}{ LOW.POP. } & \multicolumn{1}{c}{ CHARGE } \\
$1 \mathrm{~S}$ & 15.735740 & 0.264260 & 15.717735 & 0.282265 \\
2 & $\mathrm{C}$ & 6.196928 & -0.196928 & 6.135312 & -0.135312 \\
3 & $\mathrm{C}$ & 6.196922 & -0.196922 & 6.135312 & -0.135312 \\
4 & $\mathrm{C}$ & 6.139454 & -0.139454 & 6.116282 & -0.116282 \\
5 & $\mathrm{C}$ & 5.968774 & 0.031226 & 6.023915 & -0.023915 \\
6 & $\mathrm{C}$ & 6.127589 & -0.127589 & 6.114502 & -0.114502 \\
7 & $\mathrm{C}$ & 6.110192 & -0.110192 & 6.106283 & -0.106283 \\
8 & $\mathrm{C}$ & 6.096049 & -0.096049 & 6.105958 & -0.105958 \\
9 & $\mathrm{H}$ & 0.866984 & 0.133016 & 0.881638 & 0.118362 \\
10 & $\mathrm{H}$ & 0.875360 & 0.124640 & 0.886710 & 0.113290 \\
11 & $\mathrm{H}$ & 0.874722 & 0.125278 & 0.886299 & 0.113701 \\
12 & $\mathrm{H}$ & 0.876077 & 0.123923 & 0.884232 & 0.115768 \\
13 & $\mathrm{C}$ & 6.139456 & -0.139456 & 6.116283 & -0.116283 \\
14 & $\mathrm{C}$ & 6.127590 & -0.127590 & 6.114500 & -0.114500 \\
15 & $\mathrm{C}$ & 5.968778 & 0.031222 & 6.023914 & -0.023914 \\
16 & $\mathrm{C}$ & 6.096052 & -0.096052 & 6.105960 & -0.105960 \\
17 & $\mathrm{C}$ & 6.110190 & -0.110190 & 6.106283 & -0.106283 \\
18 & $\mathrm{H}$ & 0.866985 & 0.133015 & 0.881639 & 0.118361 \\
19 & $\mathrm{H}$ & 0.874722 & 0.125278 & 0.886299 & 0.113701 \\
20 & $\mathrm{H}$ & 0.875360 & 0.124640 & 0.886711 & 0.113289 \\
$21 \mathrm{H}$ & 0.876076 & 0.123924 & 0.884232 & 0.115768
\end{tabular}

\section{dimethyl selenide}

MP2 / 6-31g (d, p)

$\begin{array}{lrrrr}\mathrm{C} & 6.0 & 1.4500939122 & .0000170736 & -.0058103875 \\ \mathrm{SE} & 34.0 & -.0000000345 & -.0000822221 & -1.3051163153 \\ \mathrm{C} & 6.0 & -1.4500948675 & .0000211826 & -.0058117184 \\ \mathrm{H} & 1.0 & 1.3991811338 & -.8929683023 & .6110263873 \\ \mathrm{H} & 1.0 & 1.3993561441 & .8931970519 & .6106664843 \\ \mathrm{H} & 1.0 & 2.3828581732 & -.0002031898 & -.5633242542 \\ \mathrm{H} & 1.0 & -1.3991811236 & -.8929709347 & .6110279754 \\ \mathrm{H} & 1.0 & -2.3828573048 & -.0002032332 & -.5633236884 \\ \mathrm{H} & 1.0 & -1.3993560330 & .8931954337 & .6106655708 \\ & & & \\ \mathrm{MP} / 6-31 G(d, p) & \text { Energy } & -1554502.477643 \mathrm{KCAL} / \mathrm{MOL} \\ \mathrm{ZPE} \text { Correction } & & 58.4 & \mathrm{KCAL} / \mathrm{MOL} \\ \text { 298.15K Temperature Correction } & 52.173 & \mathrm{KCAL} / \mathrm{MOL}\end{array}$




\begin{tabular}{lcc}
\hline Basis Set & MP2 $(\mathrm{kcal} / \mathrm{mol})$ & B3LYP $(\mathrm{kcal} / \mathrm{mol})$ \\
\hline $6-31 \mathrm{G}(\mathrm{d})$ & -1554471.647425 & -1555616.774921 \\
$6-31 \mathrm{G}(2 \mathrm{~d})$ & -1554571.415981 & -1555693.317194 \\
$6-31 \mathrm{G}(\mathrm{d}, \mathrm{p})$ & -1554502.477643 & -1555622.798761 \\
$6-31+\mathrm{G}(\mathrm{d})$ & -1554485.229319 & -1555629.816120 \\
$6-31+\mathrm{G}(2 \mathrm{~d}, \mathrm{p})$ & -1554610.962258 & -1555708.541470 \\
$6-311 \mathrm{G}(\mathrm{d})$ & -1555874.429914 & -1556973.245152 \\
$6-311 \mathrm{G}(3 \mathrm{df}, 2 \mathrm{p})$ & -1555959.852014 & -1556985.741316 \\
$6-311++\mathrm{G}(\mathrm{d})$ & -1555875.525965 & -1556973.705192 \\
$6-311++\mathrm{G}(3 \mathrm{df}, 2 \mathrm{p})$ & -1555960.544073 & -1556986.234998 \\
\hline G3 (298.15K) & \multicolumn{3}{c}{-1556658.3690} \\
G3 (0K) & -1556661.1748 \\
\hline
\end{tabular}

TOTAL MULLIKEN AND LOWDIN ATOMIC POPULATIONS

\begin{tabular}{llrrr} 
ATOM & MULL.POP & \multicolumn{1}{c}{ CHARGE } & \multicolumn{1}{c}{ LOW.POP. } & \multicolumn{1}{c}{ CHARGE } \\
$1 \mathrm{C}$ & 6.419739 & -.419739 & 6.386648 & -.386648 \\
$2 \mathrm{SE}$ & 33.994745 & .005255 & 33.922328 & .077672 \\
$3 \mathrm{C}$ & 6.419739 & -.419739 & 6.386648 & -.386648 \\
$4 \mathrm{H}$ & .862700 & .137300 & .887202 & .112798 \\
$5 \mathrm{H}$ & .862682 & .137318 & .887202 & .112798 \\
$6 \mathrm{H}$ & .857506 & .142494 & .877784 & .122216 \\
$7 \mathrm{H}$ & .862697 & .137303 & .887201 & .112799 \\
$8 \mathrm{H}$ & .857505 & .142495 & .877784 & .122216 \\
$9 \mathrm{H}$ & .862686 & .137314 & .887203 & .112797
\end{tabular}

\section{selenirane}

$\operatorname{MP} 2 / 6-31 G(d, p)$

$\begin{array}{lrrrr}\mathrm{SE} & 34.0 & .0000000006 & .0000000000 & -1.7190312090 \\ \mathrm{C} & 6.0 & -.7372628793 & .0000000004 & .1098592251 \\ \mathrm{H} & 1.0 & -1.2493481973 & -.9141969100 & .3748281495 \\ \mathrm{C} & 6.0 & .7372628781 & .0000000001 & .1098592216 \\ \mathrm{H} & 1.0 & -1.2493481973 & .9141969095 & .3748281510 \\ \mathrm{H} & 1.0 & 1.2493481979 & .9141969103 & .3748281501 \\ \mathrm{H} & 1.0 & 1.2493481973 & -.9141969103 & .3748281518\end{array}$

MP2 / 6-31G (d,p) Energy

$-1553743.742 \mathrm{KCAL} / \mathrm{MOL}$ $35.04 \mathrm{KCAL} / \mathrm{MOL}$

ZPE Correction $37.809 \mathrm{KCAL} / \mathrm{MOL}$ 


\begin{tabular}{lcc}
\hline Basis Set & MP2 $(\mathrm{kcal} / \mathrm{mol})$ & B3LYP $(\mathrm{kcal} / \mathrm{mol})$ \\
\hline $6-31 \mathrm{G}(\mathrm{d})$ & -1553723.394 & -1554848.268 \\
$6-31 \mathrm{G}(2 \mathrm{~d})$ & -1553822.846 & -1554925.987 \\
$6-31 \mathrm{G}(\mathrm{d}, \mathrm{p})$ & -1553743.742 & -1554852.453 \\
$6-31+\mathrm{G}(\mathrm{d})$ & -1553738.427 & -1554862.515 \\
$6-31+\mathrm{G}(2 \mathrm{~d}, \mathrm{p})$ & -1553853.067 & -1554940.373 \\
$6-311 \mathrm{G}(\mathrm{d})$ & -1555126.673 & -1556206.323 \\
$6-311 \mathrm{G}(3 \mathrm{df}, 2 \mathrm{p})$ & -1555200.819 & -1556217.718 \\
$6-311++\mathrm{G}(\mathrm{d})$ & -1555127.672 & -1556206.769 \\
$6-311++\mathrm{G}(3 \mathrm{df}, 2 \mathrm{p})$ & -1555201.548 & -1556218.267 \\
\hline G3 (298.15K) & -1555900.7050 \\
G3 (0K) & -1555904.0878 \\
\hline
\end{tabular}

TOTAL MULLIKEN AND LOWDIN ATOMIC POPULATIONS

\begin{tabular}{|c|c|c|c|c|}
\hline ATOM & MULL . POP . & CHARGE & LOW. POP. & CHARGE \\
\hline $1 \mathrm{SE}$ & 34.107651 & -0.107651 & 34.031735 & -0.031735 \\
\hline $2 \mathrm{C}$ & 6.267231 & -0.267231 & 6.235767 & -0.235767 \\
\hline $3 \mathrm{H}$ & 0.839472 & 0.160528 & 0.874183 & 0.125817 \\
\hline $\mathrm{C}$ & 6.267231 & -0.267231 & 6.235767 & -0.235767 \\
\hline $5 \mathrm{H}$ & 0.839472 & 0.160528 & 0.874183 & 0.125817 \\
\hline $6 \mathrm{H}$ & 0.839472 & 0.160528 & 0.874183 & 0.125817 \\
\hline $7 \mathrm{H}$ & 0.839472 & 0.160528 & 0.874183 & 0.125817 \\
\hline
\end{tabular}

methyl vinyl selenide

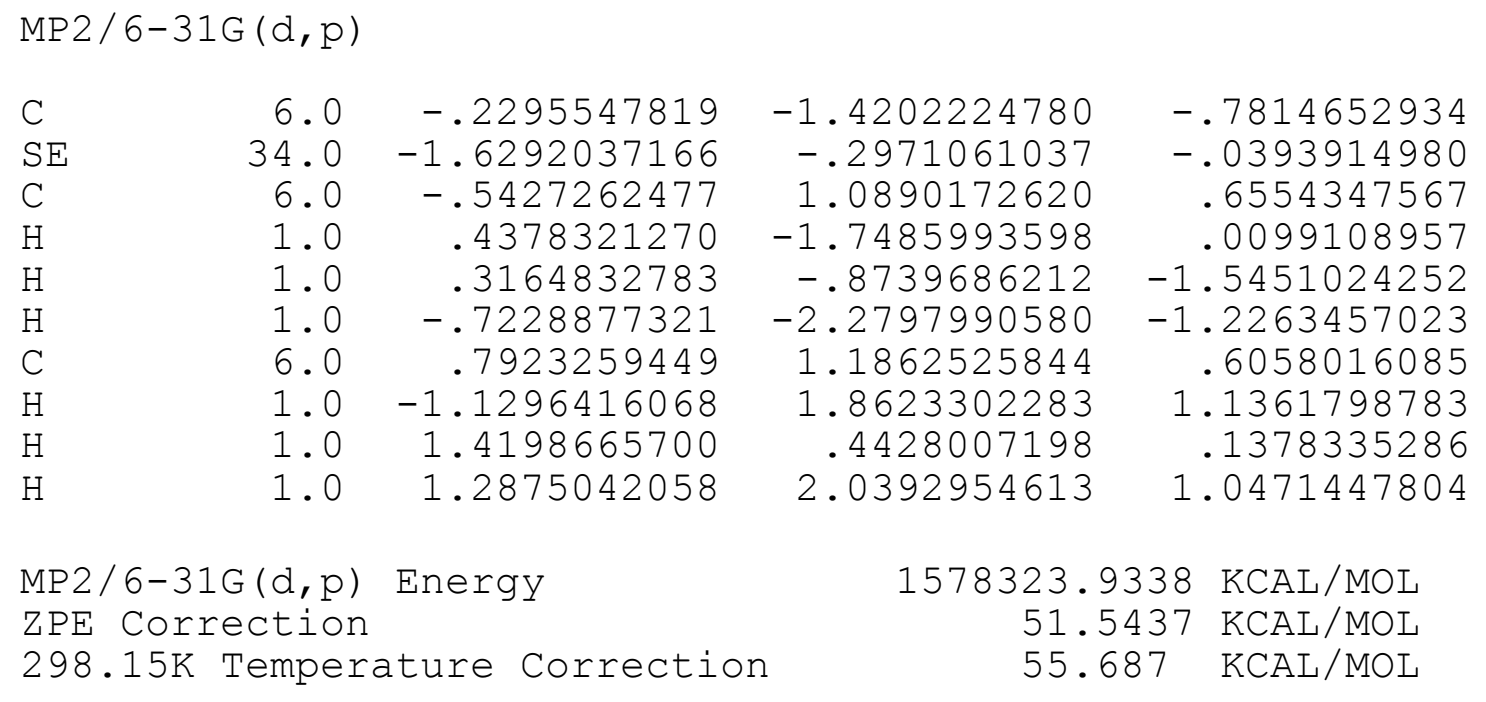




\begin{tabular}{lcc}
\hline Basis Set & MP2 $(\mathrm{kcal} / \mathrm{mol})$ & B3LYP $(\mathrm{kcal} / \mathrm{mol})$ \\
\hline $6-31 \mathrm{G}(\mathrm{d})$ & -1578293.073 & -1579496.991 \\
$6-31 \mathrm{G}(2 \mathrm{~d})$ & -1578399.927 & -1579574.889 \\
$6-31 \mathrm{G}(\mathrm{d}, \mathrm{p})$ & -1578323.936 & -1579503.306 \\
$6-31+\mathrm{G}(\mathrm{d})$ & -1578312.804 & -1579515.26 \\
$6-31+\mathrm{G}(2 \mathrm{~d}, \mathrm{p})$ & -1578443.142 & -1579594.083 \\
$6-311 \mathrm{G}(\mathrm{d})$ & -1579704.734 & -1580859.329 \\
$6-311 \mathrm{G}(3 \mathrm{df}, 2 \mathrm{p})$ & -1579804.663 & -1580874.795 \\
$6-311++\mathrm{G}(\mathrm{d})$ & -1579706.791 & -1580860.414 \\
$6-311++\mathrm{G}(3 \mathrm{df}, 2 \mathrm{p})$ & -1579806.047 & -1580875.823 \\
\hline $\mathrm{G} 3(298.15 \mathrm{~K})$ & -1580541.0580 \\
$G 3(0 \mathrm{~K})$ & -1580544.1427 \\
\hline
\end{tabular}

TOTAL MULLIKEN AND LOWDIN ATOMIC POPULATIONS

\begin{tabular}{rlrrr} 
ATOM & MULL.POP & \multicolumn{1}{c}{ CHARGE } & \multicolumn{1}{c}{ LOW.POP. } & \multicolumn{1}{c}{ CHARGE } \\
$1 \mathrm{C}$ & 6.438004 & -.438004 & 6.387679 & -.387679 \\
$2 \mathrm{SE}$ & 33.950870 & .049130 & 33.866014 & .133986 \\
$3 \mathrm{C}$ & 6.162443 & -.162443 & 6.198583 & -.198583 \\
$4 \mathrm{H}$ & .851445 & .148555 & .880799 & .119201 \\
$5 \mathrm{H}$ & .851468 & .148532 & .880383 & .119617 \\
$6 \mathrm{H}$ & .853718 & .146282 & .873592 & .126408 \\
$7 \mathrm{C}$ & 6.256105 & -.256105 & 6.266126 & -.266126 \\
$8 \mathrm{H}$ & .864299 & .135701 & .876092 & .123908 \\
$9 \mathrm{H}$ & .890834 & .109166 & .888886 & .111114 \\
$10 \mathrm{H}$ & .880813 & .119187 & .881846 & .118154
\end{tabular}

\section{selenophene}

\begin{tabular}{|c|c|c|c|c|}
\hline C & 6.0 & -1.2845599352 & .0000376878 & -.5553659411 \\
\hline & 6.0 & -.7110015139 & -.0000116293 & .6927145916 \\
\hline & 6.0 & .7110013313 & -.0000116425 & .6927155114 \\
\hline & 6.0 & 1.2845605400 & .0000376492 & -.5553666274 \\
\hline & 1.0 & -2.3363305215 & -.0000043432 & -.7905266463 \\
\hline & 1.0 & -1.3015342076 & -.0000043280 & 1.5985647623 \\
\hline & 1.0 & 1.3015341209 & -.0000044091 & 1.5985645467 \\
\hline & 1.0 & 2.3363302167 & -.0000042732 & -.7905265581 \\
\hline & 34.0 & -.0000000307 & -.0000347121 & -1.8907737452 \\
\hline & $\begin{array}{l}G(d, p) \\
\text { ection } \\
\text { Temper } \\
1 G(3 d f\end{array}$ & $\begin{array}{l}\text { ature Correction } \\
\text { 2p) Energy }\end{array}$ & \multicolumn{2}{|c|}{$\begin{array}{cl}-1601427.2925 & \mathrm{KCAL} / \mathrm{MOL} \\
41.69 & \mathrm{KCAL} / \mathrm{MOL} \\
44.988 & \mathrm{KCAL} / \mathrm{MOL} \\
-1602925.436 & \mathrm{KCAL} / \mathrm{MOL}\end{array}$} \\
\hline
\end{tabular}




\begin{tabular}{llrrrr}
\multicolumn{4}{l}{ TOTAL MULLIKEN AND LOWDIN ATOMIC } & POPULATIONS \\
ATOM & MULL.POP & CHARGE & LOW.POP. & \multicolumn{1}{c}{ CHARGE } \\
$1 \mathrm{C}$ & 6.244234 & -0.244234 & 6.247936 & -0.247936 \\
$2 \mathrm{C}$ & 6.099703 & -0.099703 & 6.140235 & -0.140235 \\
$3 \mathrm{C}$ & 6.099703 & -0.099703 & 6.140235 & -0.140235 \\
$4 \mathrm{C}$ & 6.244234 & -0.244234 & 6.247936 & -0.247936 \\
$5 \mathrm{H}$ & 0.854096 & 0.145904 & 0.873625 & 0.126375 \\
$6 \mathrm{H}$ & 0.873359 & 0.126641 & 0.880685 & 0.119315 \\
$7 \mathrm{H}$ & 0.873359 & 0.126641 & 0.880685 & 0.119315 \\
$8 \mathrm{H}$ & 0.854097 & 0.145903 & 0.873625 & 0.126375 \\
$9 \mathrm{SE}$ & 33.857215 & 0.142785 & 33.715038 & 0.284962
\end{tabular}

\section{benzoselenophene}

MP 2 / 6-31G (d, p)

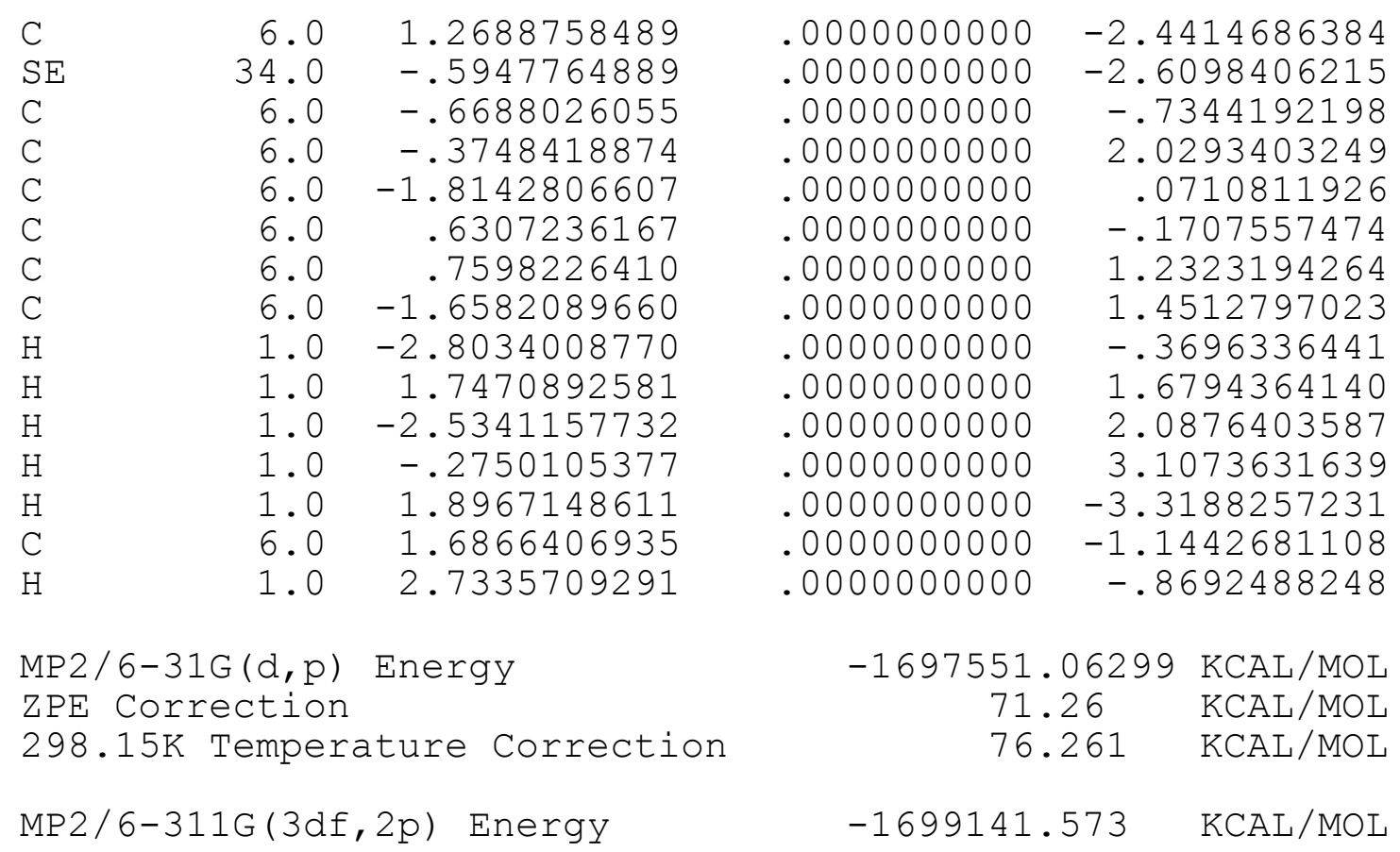




\begin{tabular}{|c|c|c|c|c|c|}
\hline $\mathrm{ATO}$ & & MULL. POP. & CHARGE & LOW. POP. & CHARGE \\
\hline & $\mathrm{C}$ & 6.245838 & -.245838 & 6.227994 & -.227994 \\
\hline 2 & $S E$ & 33.878453 & .121547 & 33.750769 & .249231 \\
\hline 3 & $\mathrm{C}$ & 6.108596 & -.108596 & 6.112733 & -.112733 \\
\hline 4 & $\mathrm{C}$ & 6.130038 & -.130038 & 6.113237 & -.113237 \\
\hline 5 & C & 6.116710 & -.116710 & 6.116599 & -.116599 \\
\hline 6 & $\mathrm{C}$ & 5.973847 & .026153 & 6.024557 & -.024557 \\
\hline 7 & C & 6.116324 & -.116324 & 6.108943 & -.108943 \\
\hline 8 & C & 6.120827 & -.120827 & 6.114691 & -.114691 \\
\hline 9 & $\mathrm{H}$ & .870762 & .129238 & .884002 & .115998 \\
\hline 10 & $\mathrm{H}$ & .876580 & .123420 & .886303 & .113697 \\
\hline 11 & $\mathrm{H}$ & .876948 & .123052 & .884445 & .115555 \\
\hline 12 & $\mathrm{H}$ & .877722 & .122278 & .888619 & .111381 \\
\hline 13 & $\mathrm{H}$ & .851344 & .148656 & .870639 & .129361 \\
\hline 14 & C & 6.086731 & -.086731 & 6.135151 & -.135151 \\
\hline 15 & $\mathrm{H}$ & .869279 & .130721 & .881317 & .118683 \\
\hline
\end{tabular}

\section{dibenzoselenophene}

MP2 / 6-31G (d, p)

\begin{tabular}{|c|c|c|c|c|c|}
\hline $\mathrm{H}$ & 1.0 & -3.1172621713 & -.0001441883 & \multicolumn{2}{|c|}{-2.3316431483} \\
\hline $\mathrm{C}$ & 6.0 & -2.6871622640 & -.0000848793 & \multicolumn{2}{|c|}{-1.3377930002} \\
\hline $\mathrm{C}$ & 6.0 & -1.5763147720 & .0000818764 & \multicolumn{2}{|c|}{1.2507529306} \\
\hline C & 6.0 & -3.5089325298 & -.0001010718 & \multicolumn{2}{|c|}{-.2153408270} \\
\hline C & 6.0 & -1.3005900034 & .0000536347 & \multicolumn{2}{|c|}{-1.1579113148} \\
\hline C & 6.0 & -.7260534951 & .0001039482 & \multicolumn{2}{|c|}{.1320974441} \\
\hline $\mathrm{C}$ & 6.0 & -2.9544873569 & .0000905484 & \multicolumn{2}{|c|}{1.0745039515} \\
\hline $\mathrm{H}$ & 1.0 & -4.5845034781 & -.0001815380 & \multicolumn{2}{|c|}{-.3395084726} \\
\hline SE & 34.0 & .000003 & -.0000470427 & \multicolumn{2}{|c|}{-2.5276972629} \\
\hline $\mathrm{H}$ & 1.0 & -3.6066549646 & .0000209037 & \multicolumn{2}{|c|}{1.9384689218} \\
\hline $\mathrm{H}$ & 1.0 & -1.1578729463 & .0001896909 & \multicolumn{2}{|c|}{2.2502203299} \\
\hline C & 6.0 & 1.300 & -.0000121722 & \multicolumn{2}{|c|}{-1.1579631094} \\
\hline $\mathrm{C}$ & 6.0 & 2.9545113122 & -.0000063661 & \multicolumn{2}{|c|}{1.044657138} \\
\hline C & 6.0 & 260239797 & .00009 & \multicolumn{2}{|c|}{.1321457426} \\
\hline $\mathrm{C}$ & 6.0 & $2.68^{\circ}$ & -.00003 & \multicolumn{2}{|c|}{-1.3378263549} \\
\hline $\mathrm{C}$ & 6.0 & 3.508 & -.0001187288 & \multicolumn{2}{|c|}{-.2152771724} \\
\hline $\mathrm{C}$ & 6.0 & 1.576 & .0001773701 & \multicolumn{2}{|c|}{1.2507737007} \\
\hline $\mathrm{H}$ & 1.0 & 3.11 & -.00013 & \multicolumn{2}{|c|}{-2.3316362832} \\
\hline $\mathrm{H}$ & 1.0 & 4.58 & -.0001 & \multicolumn{2}{|c|}{-.3395109379} \\
\hline $\mathrm{H}$ & 1.0 & & & \multicolumn{2}{|c|}{2.2502093556} \\
\hline $\mathrm{H}$ & 1.0 & 3.60665 & 570 & \multicolumn{2}{|c|}{1.9384697382} \\
\hline \multicolumn{3}{|c|}{ MP2 / 6-31G (d,p) Energy } & \multicolumn{3}{|c|}{-1793677.5472} \\
\hline & ection & & & $\begin{array}{l}5472 \\
19\end{array}$ & $\mathrm{KCAL} / \mathrm{MOL}$ \\
\hline \multicolumn{3}{|c|}{ 298.15K Temperature Correction } & 107.422 & $\begin{array}{l}9 \\
22\end{array}$ & $\mathrm{KCAL} / \mathrm{MO}$ \\
\hline \multicolumn{3}{|c|}{ MP2/6-311G (3df, 2p) Energy } & \multicolumn{2}{|c|}{-1795362.888} & \\
\hline
\end{tabular}




\begin{tabular}{|c|c|c|c|c|}
\hline ATOM & MULL. POP. & CHARGE & LOW. POP. & CHARGE \\
\hline $1 \mathrm{H}$ & 0.868926 & 0.131074 & 0.883394 & 0.116606 \\
\hline $\mathrm{C}$ & 6.126196 & -0.126196 & 6.116695 & -0.116695 \\
\hline $\mathrm{C}$ & 6.100671 & -0.100671 & 6.106021 & -0.106021 \\
\hline $\mathrm{C}$ & 6.113275 & -0.113275 & 6.105939 & -0.105939 \\
\hline $\mathrm{C}$ & 6.105219 & -0.105219 & 6.099037 & -0.099037 \\
\hline $\mathrm{C}$ & 5.978209 & 0.021791 & 6.022551 & -0.022551 \\
\hline $\mathrm{C}$ & 6.137262 & -0.137262 & 6.115426 & -0.115426 \\
\hline $\mathrm{H}$ & 0.874900 & 0.125100 & 0.886860 & 0.113140 \\
\hline $9 \mathrm{SE}$ & 33.889185 & 0.110815 & 33.786700 & 0.213300 \\
\hline $10 \mathrm{H}$ & 0.875758 & 0.124242 & 0.884289 & 0.115711 \\
\hline 11 & 0.874949 & 0.125051 & 0.886432 & 0.113568 \\
\hline 12 & 6.105058 & -0.105058 & 6.099040 & -0.099040 \\
\hline 13 & 6.137275 & -0.137275 & 6.115424 & -0.115424 \\
\hline 14 & 5.978443 & 0.021557 & 6.022544 & -0.022544 \\
\hline 15 & 6.126285 & -0.126285 & 6.116708 & -0.116708 \\
\hline 16 & 6.113240 & -0.113240 & 6.105938 & -0.105938 \\
\hline 17 & 6.100605 & -0.100605 & 6.106025 & -0.106025 \\
\hline $18 \mathrm{H}$ & 0.868927 & 0.131073 & 0.883393 & 0.116607 \\
\hline 19 & 0.874903 & 0.125097 & 0.886860 & 0.113140 \\
\hline 20 & 0.874948 & 0.125052 & 0.886432 & 0.113568 \\
\hline $21 \mathrm{H}$ & 0.875763 & 0.124237 & 0.884291 & 0.115709 \\
\hline
\end{tabular}

\section{dimethyl sulfoxide (1)}

$\operatorname{MP} 2 / 6-31 G(d, p)$

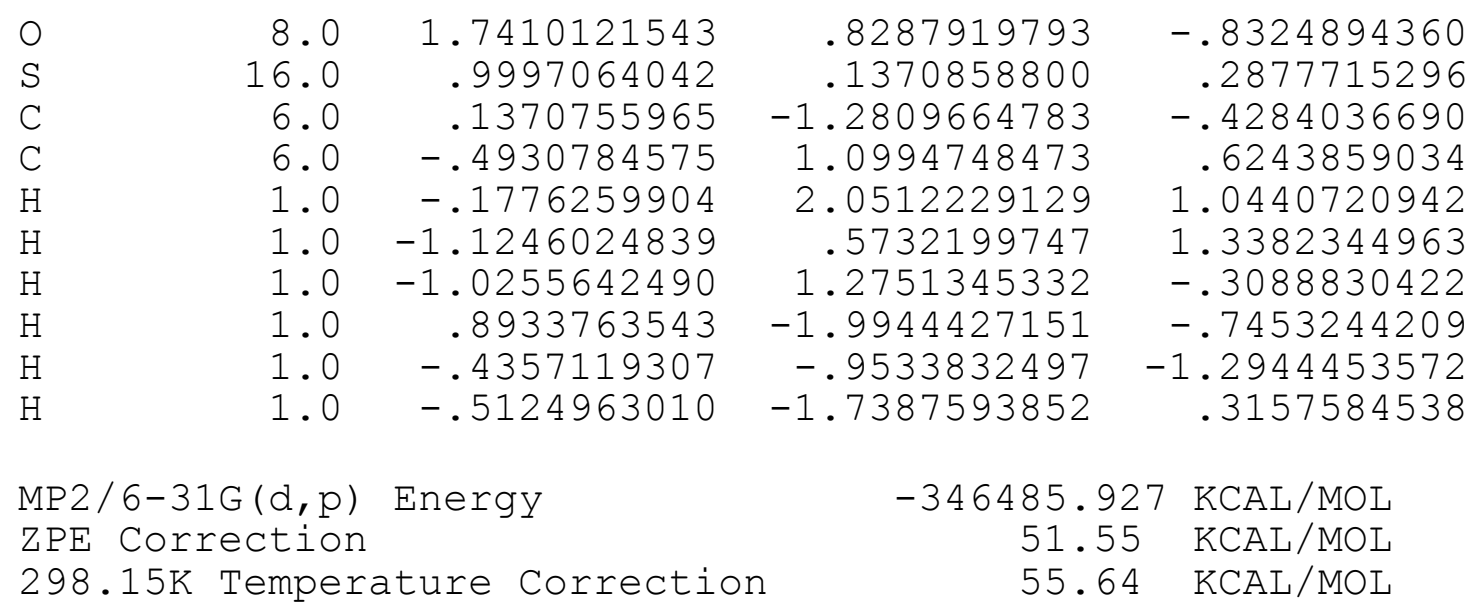




\begin{tabular}{lcc}
\hline Basis Set & MP2 $(\mathrm{kcal} / \mathrm{mol})$ & B3LYP $(\mathrm{kcal} / \mathrm{mol})$ \\
\hline $6-31 \mathrm{G}(\mathrm{d})$ & -346455.9582 & -347031.2884 \\
$6-31 \mathrm{G}(2 \mathrm{~d})$ & -346496.7756 & -347037.0735 \\
$6-31 \mathrm{G}(\mathrm{d}, \mathrm{p})$ & -346485.927 & -347036.7247 \\
$6-31+\mathrm{G}(\mathrm{d})$ & -346466.968 & -347039.552 \\
$6-31+\mathrm{G}(2 \mathrm{~d}, \mathrm{p})$ & -346537.8025 & -347053.5119 \\
$6-311 \mathrm{G}(\mathrm{d})$ & -346522.0576 & -347074.2171 \\
$6-311 \mathrm{G}(3 \mathrm{df}, 2 \mathrm{p})$ & -346657.4402 & -347104.9657 \\
$6-311++\mathrm{G}(\mathrm{d})$ & -346529.8094 & -347079.2778 \\
$6-311++\mathrm{G}(3 \mathrm{df}, 2 \mathrm{p})$ & -346661.8254 & -347108.5033 \\
\hline G3 (298.15K) & -346969.1683 \\
$\mathrm{G} 3(0 \mathrm{~K})$ & -346972.7112 \\
\hline
\end{tabular}

\begin{tabular}{|c|c|c|c|c|}
\hline TOTAL & MULLIKEN AND & & & \\
\hline ATOM & MULL . POP . & CHARGE & LOW. POP. & CHARGE \\
\hline 10 & 8.659566 & -.659566 & 8.695232 & -.695232 \\
\hline $\mathrm{S}$ & 15.182643 & .817357 & 15.141645 & .858355 \\
\hline C & 6.530981 & -.530981 & 6.463207 & -.463207 \\
\hline $\mathrm{C}$ & 6.530978 & -.530978 & 6.462249 & -.462249 \\
\hline $\mathrm{H}$ & .838406 & .161594 & .867794 & .132206 \\
\hline $\mathrm{H}$ & .864918 & 082 & .875256 & .124744 \\
\hline $7 \mathrm{H}$ & .844593 & .155407 & .876511 & .123489 \\
\hline $8 \mathrm{H}$ & .838404 & .161596 & .866770 & .133230 \\
\hline $9 \mathrm{H}$ & .844599 & 401 & .875739 & .124261 \\
\hline $10 \mathrm{H}$ & .864913 & .13 & .875598 & .124402 \\
\hline
\end{tabular}

\section{diethyl sulfoxide (2)}

MP2 / 6-31G (d,p)

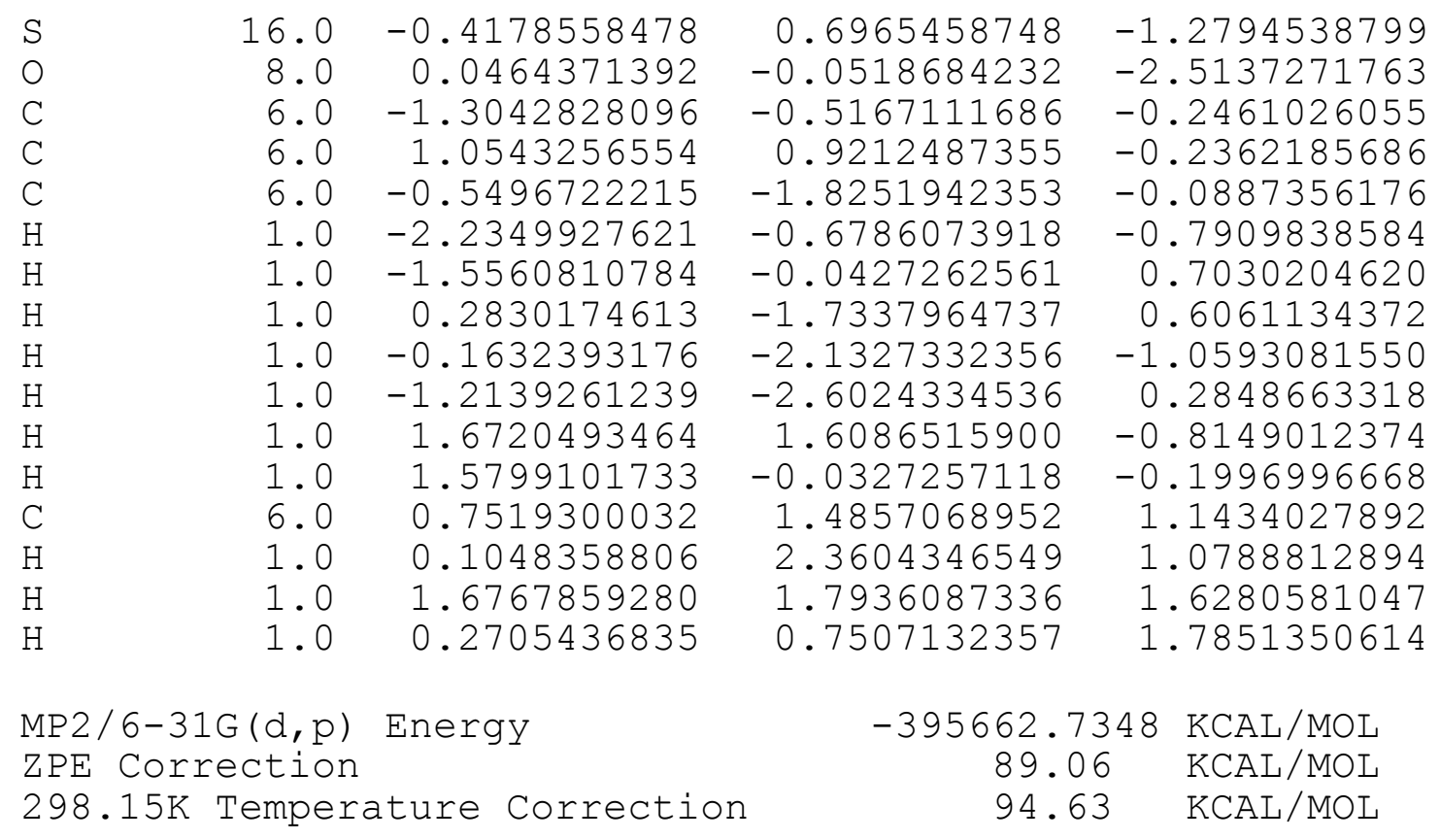




\begin{tabular}{lcc}
\hline Basis Set & MP2 $(\mathrm{kcal} / \mathrm{mol})$ & B3LYP $(\mathrm{kcal} / \mathrm{mol})$ \\
\hline $6-31 \mathrm{G}(\mathrm{d})$ & -395611.9249 & -396334.9591 \\
$6-31 \mathrm{G}(2 \mathrm{~d})$ & -395663.9647 & -396339.9792 \\
$6-31 \mathrm{G}(\mathrm{d}, \mathrm{p})$ & -395662.7348 & -396343.9067 \\
$6-31+\mathrm{G}(\mathrm{d})$ & -395624.3657 & -396343.637 \\
$6-31+\mathrm{G}(2 \mathrm{~d}, \mathrm{p})$ & -395725.5519 & -396360.734 \\
$6-311 \mathrm{G}(\mathrm{d})$ & -395699.3273 & -396389.5268 \\
$6-311 G(3 \mathrm{df}, 2 \mathrm{p})$ & -395882.3701 & -396425.2243 \\
$6-311++G(\mathrm{~d})$ & -395706.7625 & -396393.6095 \\
$6-311++G(3 \mathrm{df}, 2 \mathrm{p})$ & -395886.5474 & -396428.3348 \\
\hline G3 (298.15K) & -396254.3465 \\
G3 (0K) & -396259.5768 \\
\hline
\end{tabular}

TOTAL MULLIKEN AND LOWDIN ATOMIC POPULATIONS

\begin{tabular}{rrrrrr} 
ATOM & MULL.POP. & \multicolumn{1}{c}{ CHARGE } & \multicolumn{1}{l}{ LOW.POP. } & \multicolumn{1}{l}{ CHARGE } \\
1 & $\mathrm{~S}$ & 15.174507 & 0.825493 & 15.164532 & 0.835468 \\
2 & $\mathrm{O}$ & 8.676491 & -0.676491 & 8.696827 & -0.696827 \\
3 & $\mathrm{C}$ & 6.420020 & -0.420020 & 6.342271 & -0.342271 \\
4 & $\mathrm{C}$ & 6.415316 & -0.415316 & 6.341528 & -0.341528 \\
5 & $\mathrm{C}$ & 6.352919 & -0.352919 & 6.312322 & -0.312322 \\
6 & $\mathrm{H}$ & 0.843190 & 0.156810 & 0.875351 & 0.124649 \\
7 & $\mathrm{H}$ & 0.864718 & 0.135282 & 0.884203 & 0.115797 \\
8 & $\mathrm{H}$ & 0.885761 & 0.114239 & 0.896145 & 0.103855 \\
9 & $\mathrm{H}$ & 0.838231 & 0.161769 & 0.875838 & 0.124162 \\
10 & $\mathrm{H}$ & 0.872206 & 0.127794 & 0.885039 & 0.114961 \\
11 & $\mathrm{H}$ & 0.842984 & 0.157016 & 0.872601 & 0.127399 \\
12 & $\mathrm{H}$ & 0.842653 & 0.157347 & 0.881815 & 0.118185 \\
13 & $\mathrm{C}$ & 6.362758 & -0.362758 & 6.310577 & -0.310577 \\
14 & $\mathrm{H}$ & 0.868246 & 0.131754 & 0.887963 & 0.112037 \\
15 & $\mathrm{H}$ & 0.864217 & 0.135783 & 0.881984 & 0.118016 \\
16 & $\mathrm{H}$ & 0.875782 & 0.124218 & 0.891003 & 0.108997
\end{tabular}




\section{allyl ethyl sulfoxide (3)}

MP2 / 6-31G (d, p)

$\begin{array}{lrrrr}\mathrm{C} & 6.0 & 0.3808459216 & 0.7239467196 & -1.0006904390 \\ \mathrm{C} & 6.0 & 0.8611342750 & -0.6665228313 & -1.2473848012 \\ \mathrm{C} & 6.0 & 2.1407425601 & -0.9691068617 & -1.5061089150 \\ \mathrm{H} & 1.0 & 0.1313818574 & -1.4644566891 & -1.1678393949 \\ \mathrm{H} & 1.0 & 2.8948877454 & -0.1976124640 & -1.5918818052 \\ \mathrm{H} & 1.0 & 2.4606295102 & -1.9909504441 & -1.6497052707 \\ \mathrm{H} & 1.0 & -0.6625362456 & 0.8799286828 & -1.2734948027 \\ \mathrm{H} & 1.0 & 0.9833294904 & 1.4594012175 & -1.5344232683 \\ \mathrm{~S} & 16.0 & 0.5438113797 & 1.2170277288 & 0.7621124340 \\ \mathrm{C} & 6.0 & -0.4450320115 & -0.1330695789 & 1.4876224886 \\ \mathrm{O} & 8.0 & -0.2657329379 & 2.4848635913 & 0.9364372296 \\ \mathrm{H} & 1.0 & 0.0485224012 & -1.0762516270 & 1.2522574759 \\ \mathrm{C} & 6.0 & -1.9034092844 & -0.0869475258 & 1.0670000014 \\ \mathrm{H} & 1.0 & -0.3545538612 & 0.0330615746 & 2.5618276565 \\ \mathrm{H} & 1.0 & -2.5027830541 & -0.7285600434 & 1.7102740912 \\ \mathrm{H} & 1.0 & -2.0420012795 & -0.4197191371 & 0.0397849851 \\ \mathrm{H} & 1.0 & -2.2692363668 & 0.9349678477 & 1.1542124849\end{array}$

MP2 / 6-31G (d,p) Energy

$-419484.5513 \mathrm{KCAL} / \mathrm{MOL}$ $91.86 \mathrm{KCAL} / \mathrm{MOL}$ ZPE Correction $97.96 \mathrm{KCAL} / \mathrm{MOL}$

\begin{tabular}{lcc}
\hline Basis Set & MP2 $(\mathrm{kcal} / \mathrm{mol})$ & B3LYP $(\mathrm{kcal} / \mathrm{mol})$ \\
\hline $6-31 \mathrm{G}(\mathrm{d})$ & -419433.8215 & -420215.2447 \\
$6-31 \mathrm{G}(2 \mathrm{~d})$ & -419491.6022 & -420220.7753 \\
$6-31 \mathrm{G}(\mathrm{d}, \mathrm{p})$ & -419484.5513 & -420224.5583 \\
$6-31+\mathrm{G}(\mathrm{d})$ & -419448.9117 & -420225.5565 \\
$6-31+\mathrm{G}(2 \mathrm{~d}, \mathrm{p})$ & -419554.7592 & -420243.5759 \\
$6-311 \mathrm{G}(\mathrm{d})$ & -419529.9949 & -420275.8789 \\
$6-311 \mathrm{G}(3 \mathrm{df}, 2 \mathrm{p})$ & -419726.9787 & -420314.3251 \\
$6-311++\mathrm{G}(\mathrm{d})$ & -419538.5587 & -420280.4411 \\
$6-311++\mathrm{G}(3 \mathrm{df}, 2 \mathrm{p})$ & -419731.6825 & -420317.8096 \\
\hline G3 (298.15K) & \multicolumn{2}{c}{-420136.4879} \\
G3 (0K) & -420142.2039 \\
\hline
\end{tabular}




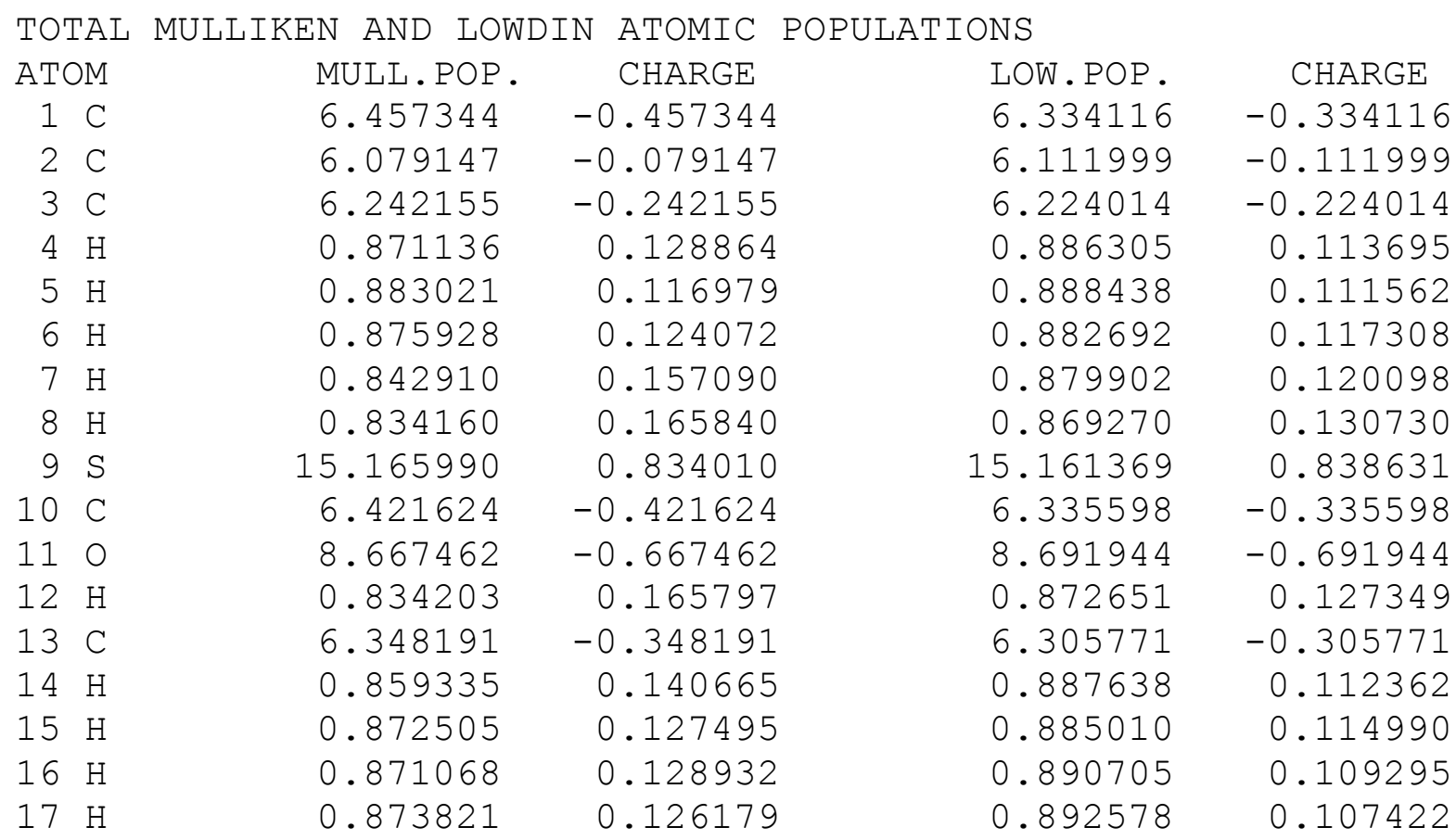

\section{diphenyl sulfoxide (4)}

$\begin{array}{lrrrr}\text { MP2 / }-31 G(d, p) & & & \\ & & & & \\ \mathrm{O} & 8.0 & -.4889894138 & 1.4224244421 & 2.5874639095 \\ \mathrm{~S} & 16.0 & -.4428551985 & 1.6897494513 & 1.0967079359 \\ \mathrm{C} & 6.0 & -1.4796817188 & .4284164649 & .3177132029 \\ \mathrm{C} & 6.0 & 1.1160999638 & 1.0055456369 & .4923111809 \\ \mathrm{C} & 6.0 & -3.1078491690 & -1.5161160453 & -.8026999595 \\ \mathrm{C} & 6.0 & -1.7767373201 & .4908256893 & -1.0447915803 \\ \mathrm{C} & 6.0 & -2.0029947738 & -.5715648496 & 1.1337552888 \\ \mathrm{C} & 6.0 & -2.8172120606 & -1.5511179743 & .5627952237 \\ \mathrm{C} & 6.0 & -2.5890771565 & -.4954353960 & -1.6043906301 \\ \mathrm{H} & 1.0 & -1.3833175189 & 1.2907322383 & -1.6610200297 \\ \mathrm{H} & 1.0 & -1.7692593794 & -.5583855774 & 2.1910838985 \\ \mathrm{H} & 1.0 & -3.2299368236 & -2.3365060188 & 1.1834390064 \\ \mathrm{H} & 1.0 & -2.8255297311 & -.4620233434 & -2.6604135555 \\ \mathrm{H} & 1.0 & -3.7412165927 & -2.2766516917 & -1.2408721652 \\ \mathrm{C} & 6.0 & 3.5241995855 & -.0574677522 & -.3758596592 \\ \mathrm{C} & 6.0 & 1.8318432503 & .1640002334 & 1.3412729116 \\ \mathrm{C} & 6.0 & 1.5956598653 & 1.3497533044 & -.7722052581 \\ \mathrm{C} & 6.0 & 2.8038143148 & .8036313776 & -1.2076598967 \\ \mathrm{C} & 6.0 & 3.0406089372 & -.3722751882 & .8967128841 \\ \mathrm{H} & 1.0 & 1.4370437062 & -.0418517657 & 2.3285795857 \\ \mathrm{H} & 1.0 & 1.0448845832 & 2.0376863018 & -1.4031885891 \\ \mathrm{H} & 1.0 & 3.1881788674 & 1.0596806585 & -2.1868567510 \\ \mathrm{H} & 1.0 & 3.6079752199 & -1.0300383444 & 1.5430840828 \\ \mathrm{H} & 1.0 & 4.4643588804 & -.4729485625 & -.7149271173\end{array}$


MP2 / 6-31G (d, p) Energy

ZPE Correction

298.15K Temperature Correction
$-586386.3836 \mathrm{KCAL} / \mathrm{MOL}$

117.32

125.47
$\mathrm{KCAL} / \mathrm{MOL}$

$\mathrm{KCAL} / \mathrm{MOL}$

\begin{tabular}{lcc}
\hline Basis Set & MP2 $(\mathrm{kcal} / \mathrm{mol})$ & B3LYP $(\mathrm{kcal} / \mathrm{mol})$ \\
\hline $6-31 \mathrm{G}(\mathrm{d})$ & -586336.6831 & -587513.5234 \\
$6-31 \mathrm{G}(2 \mathrm{~d})$ & -586432.5834 & -587522.7935 \\
$6-31 \mathrm{G}(\mathrm{d}, \mathrm{p})$ & -586386.3836 & -587523.6627 \\
$6-31+\mathrm{G}(\mathrm{d})$ & -586362.1428 & -587528.4624 \\
$6-31+\mathrm{G}(2 \mathrm{~d}, \mathrm{p})$ & -586501.9876 & -587550.8152 \\
$6-311 \mathrm{G}(\mathrm{d})$ & -586488.3678 & -587608.4153 \\
$6-311 \mathrm{G}(3 \mathrm{df}, 2 \mathrm{p})$ & -586784.7574 & -587663.4424 \\
$6-311++\mathrm{G}(\mathrm{d})$ & -586500.5452 & -587613.6807 \\
$6-311++\mathrm{G}(3 \mathrm{df}, 2 \mathrm{p})$ & -586790.4382 & -587667.1162 \\
\hline
\end{tabular}

TOTAL MULLIKEN AND LOWDIN ATOMIC POPULATIONS

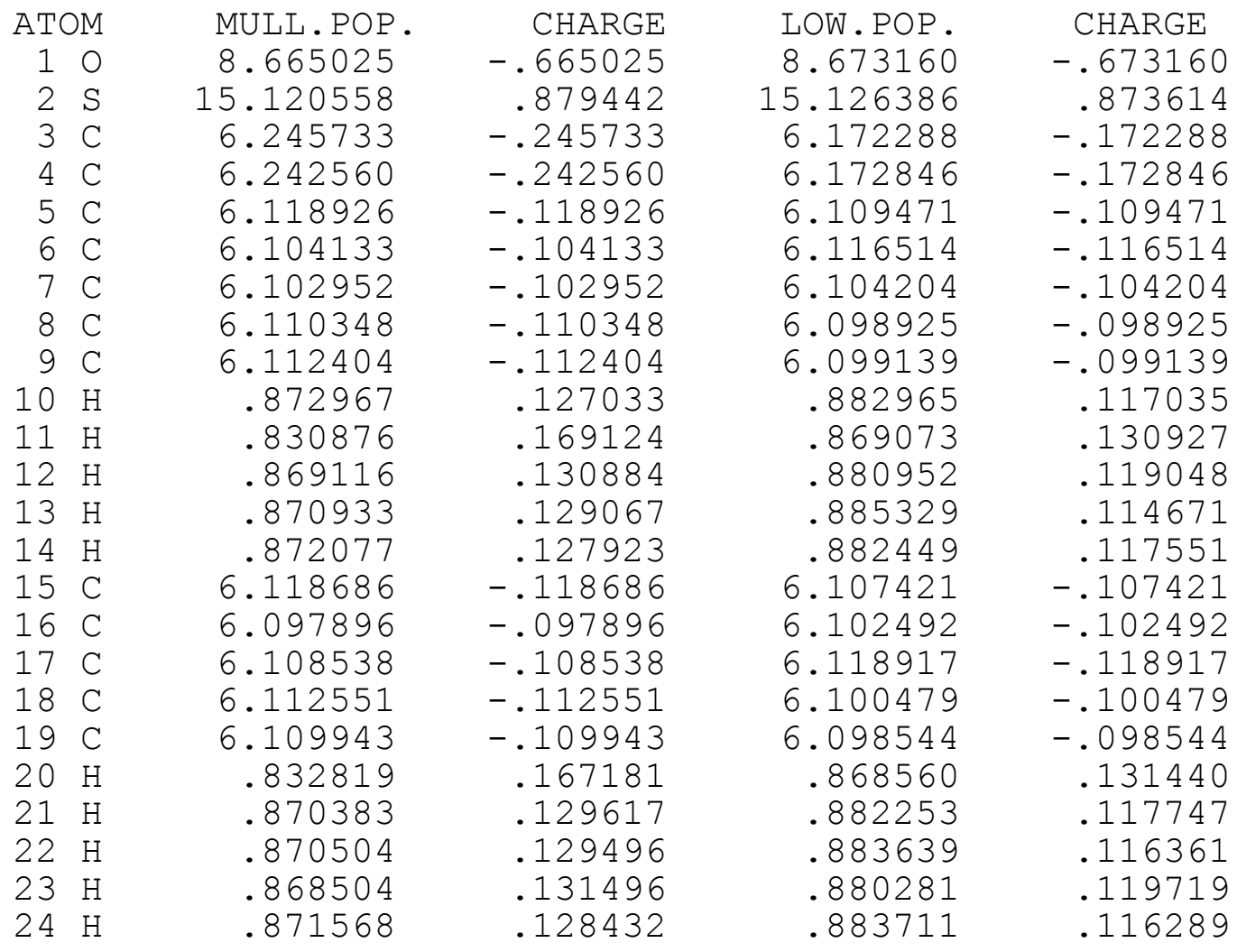




\section{thiirane oxide (5)}

$\operatorname{MP} 2 / 6-31 G(d, p)$

$\begin{array}{rrrrr}\mathrm{C} & 6.0 & -.5829264165 & .0408975030 & -1.2116099442 \\ \mathrm{C} & 6.0 & .8918715981 & .0408741821 & -1.0061651218 \\ \mathrm{H} & 1.0 & -1.0816868200 & 1.0007704394 & -1.2378994219 \\ \mathrm{H} & 1.0 & -1.0353018690 & -.7414920381 & -1.8063924729 \\ \mathrm{H} & 1.0 & 1.3788846504 & 1.0007191781 & -.8951194184 \\ \mathrm{H} & 1.0 & 1.4895511907 & -.7415460820 & -1.4546454602 \\ \mathrm{~S} & 16.0 & -.0668101573 & -.4233045020 & .4795112166 \\ \mathrm{O} & 8.0 & -.2013441764 & .7215373195 & 1.4455006227\end{array}$

MP2 / 6-31G (d,p) Energy

$-345725.4984 \mathrm{KCAL} / \mathrm{MOL}$

ZPE Correction

$37.60 \mathrm{KCAL} / \mathrm{MOL}$

298.15K Temperature Correction

$40.853 \mathrm{KCAL} / \mathrm{MOL}$

\begin{tabular}{lcc}
\hline Basis Set & MP2 $(\mathrm{kcal} / \mathrm{mol})$ & B3LYP $(\mathrm{kcal} / \mathrm{mol})$ \\
\hline $6-31 G(\mathrm{~d})$ & -345705.7661 & -346262.215 \\
$6-31 \mathrm{G}(2 \mathrm{~d})$ & -345744.3994 & -346268.1301 \\
$6-31 \mathrm{G}(\mathrm{d}, \mathrm{p})$ & -345725.4984 & -346265.8984 \\
$6-31+\mathrm{G}(\mathrm{d})$ & -345716.7609 & -346270.4567 \\
$6-31+\mathrm{G}(2 \mathrm{~d}, \mathrm{p})$ & -345774.4986 & -346282.0894 \\
$6-311 \mathrm{G}(\mathrm{d})$ & -345770.205 & -346304.699 \\
$6-311 \mathrm{G}(3 \mathrm{df}, 2 \mathrm{p})$ & -345894.7514 & -346333.641 \\
$6-311++\mathrm{G}(\mathrm{d})$ & -345777.6915 & -346309.6715 \\
$6-311++\mathrm{G}(3 \mathrm{df}, 2 \mathrm{p})$ & -345900.4427 & -346336.9871 \\
\hline G3 (298.15K) & -346207.9256 \\
G3 (0K) & -346210.6129 \\
\hline
\end{tabular}

TOTAL MULLIKEN AND LOWDIN ATOMIC POPULATIONS

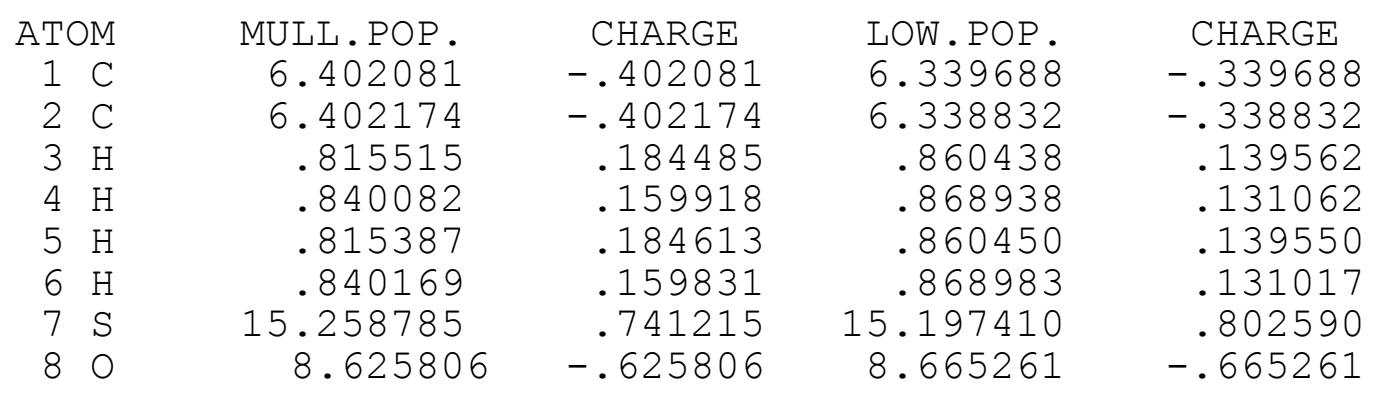


methyl vinyl sulfoxide (6)

$\operatorname{MP} 2 / 6-31 G(d, p)$

$\begin{array}{lrrrr}\mathrm{C} & 6.0 & 0.8425123786 & -0.4684376445 & 0.4572211372 \\ \mathrm{C} & 6.0 & 1.3869592096 & -1.2703555370 & -0.4593228711 \\ \mathrm{H} & 1.0 & 1.7777758662 & -2.2424564226 & -0.1970371129 \\ \mathrm{H} & 1.0 & 1.4364058052 & -0.9428085435 & -1.4894459914 \\ \mathrm{H} & 1.0 & 0.7700926580 & -0.6991989126 & 1.5133199051 \\ \mathrm{~S} & 16.0 & 0.2722033539 & 1.1518277236 & -0.0416956383 \\ \mathrm{C} & 6.0 & -1.4607220560 & 0.8503701879 & 0.3842838585 \\ \mathrm{O} & 8.0 & 0.3678152037 & 1.2193493576 & -1.5491314001 \\ \mathrm{H} & 1.0 & -1.8302973026 & 0.0266732818 & -0.2228846243 \\ \mathrm{H} & 1.0 & -2.0176328903 & 1.7568329580 & 0.1599202415 \\ \mathrm{H} & 1.0 & -1.5451102691 & 0.6182057203 & 1.4447750358\end{array}$

MP2 / 6-31G (d,p) Energy

ZPE Correction

$\begin{array}{cc}-370307.8587 & \mathrm{KCAL} / \mathrm{MOL} \\ 54.67 & \mathrm{KCAL} / \mathrm{MOL} \\ 59.141 & \mathrm{KCAL} / \mathrm{MOL}\end{array}$

298.15K Temperature Correction

\begin{tabular}{lcc}
\hline Basis Set & MP2 $(\mathrm{kcal} / \mathrm{mol})$ & B3LYP $(\mathrm{kcal} / \mathrm{mol})$ \\
\hline $6-31 G(\mathrm{~d})$ & -370277.8258 & -370910.7708 \\
$6-31 G(2 \mathrm{~d})$ & -370324.1876 & -370917.0688 \\
$6-31 \mathrm{G}(\mathrm{d}, \mathrm{p})$ & -370307.8587 & -370916.5633 \\
$6-31+\mathrm{G}(\mathrm{d})$ & -370290.3638 & -370919.8062 \\
$6-31+\mathrm{G}(2 \mathrm{~d}, \mathrm{p})$ & -370365.781 & -370934.5607 \\
$6-311 \mathrm{G}(\mathrm{d})$ & -370352.718 & -370959.9608 \\
$6-311 \mathrm{G}(3 \mathrm{df}, 2 \mathrm{p})$ & -370501.4559 & -370992.9827 \\
$6-311++\mathrm{G}(\mathrm{d})$ & -370360.3396 & -370964.4806 \\
$6-311++\mathrm{G}(3 \mathrm{df}, 2 \mathrm{p})$ & -370505.6401 & -370996.2293 \\
\hline G3 (298.15K) & -370850.1639 \\
G3 (0K) & -370854.1291 \\
\hline
\end{tabular}

TOTAL MULLIKEN AND LOWDIN ATOMIC POPULATIONS

$\begin{array}{rrrrrr}\text { ATOM } & \text { MULL.POP. } & \text { CHARGE } & \text { LOW.POP. } & \text { CHARGE } \\ 1 & \mathrm{C} & 6.322260 & -0.322260 & 6.288031 & -0.288031 \\ 2 & \mathrm{C} & 6.228335 & -0.228335 & 6.203666 & -0.203666 \\ 3 & \mathrm{H} & 0.868638 & 0.131362 & 0.878284 & 0.121716 \\ 4 & \mathrm{H} & 0.832762 & 0.167238 & 0.866614 & 0.133386 \\ 5 & \mathrm{H} & 0.855669 & 0.144331 & 0.876079 & 0.123921 \\ 6 & \mathrm{~S} & 15.165770 & 0.834230 & 15.131791 & 0.868209 \\ 7 & \mathrm{C} & 6.531090 & -0.531090 & 6.453874 & -0.453874 \\ 8 & \mathrm{O} & 8.656263 & -0.656263 & 8.684531 & -0.684531 \\ 9 & \mathrm{H} & 0.834710 & 0.165290 & 0.870131 & 0.129869 \\ 10 & \mathrm{H} & 0.840767 & 0.159233 & 0.869424 & 0.130576 \\ 11 & \mathrm{H} & 0.863736 & 0.136264 & 0.877576 & 0.122424\end{array}$




\section{thiophene-S-oxide (7)}

$\operatorname{MP} 2 / 6-31 G(d, p)$

$\begin{array}{rrrrr}\mathrm{C} & 6.0 & -0.0375150337 & 1.2567679972 & -0.2455906399 \\ \mathrm{C} & 6.0 & 0.1087333374 & 0.7265915985 & 0.9940768542 \\ \mathrm{C} & 6.0 & 0.1087336956 & -0.7265851878 & 0.9940645294 \\ \mathrm{C} & 6.0 & -0.0375250373 & -1.2567537315 & -0.2455622101 \\ \mathrm{H} & 1.0 & -0.1006437957 & 2.2954186542 & -0.5280786800 \\ \mathrm{H} & 1.0 & 0.1997311760 & 1.3166069014 & 1.8953366139 \\ \mathrm{H} & 1.0 & 0.1997147104 & -1.3166151632 & 1.8953227094 \\ \mathrm{H} & 1.0 & -0.1006480551 & -2.2954065450 & -0.5280733280 \\ \mathrm{~S} & 16.0 & -0.4605853975 & -0.0000546075 & -1.4129079122 \\ \mathrm{O} & 8.0 & 0.3078191598 & 0.0000300825 & -2.7137399298\end{array}$

MP2 / 6-31G (d,p) Energy

ZPE Correction

$-393390.8183 \mathrm{KCAL} / \mathrm{MOL}$ $44.47 \mathrm{KCAL} / \mathrm{MOL}$

298.15K Temperature Correction

$48.351 \mathrm{KCAL} / \mathrm{MOL}$

\begin{tabular}{lcc}
\hline Basis Set & MP2 $(\mathrm{kcal} / \mathrm{mol})$ & B3LYP $(\mathrm{kcal} / \mathrm{mol})$ \\
\hline $6-31 \mathrm{G}(\mathrm{d})$ & -393371.0323 & -394042.0675 \\
$6-31 \mathrm{G}(2 \mathrm{~d})$ & -393421.7221 & -394049.1976 \\
$6-31 \mathrm{G}(\mathrm{d}, \mathrm{p})$ & -393390.8183 & -394046.2208 \\
$6-31+\mathrm{G}(\mathrm{d})$ & -393384.353 & -394051.0861 \\
$6-31+\mathrm{G}(2 \mathrm{~d}, \mathrm{p})$ & -393453.9898 & -394065.2401 \\
$6-311 \mathrm{G}(\mathrm{d})$ & -393450.0461 & -394093.4536 \\
$6-311 \mathrm{G}(3 \mathrm{df}, 2 \mathrm{p})$ & -393603.404 & -394128.9835 \\
$6-311++\mathrm{G}(\mathrm{d})$ & -393459.3304 & -394099.226 \\
$6-311++\mathrm{G}(3 \mathrm{df}, 2 \mathrm{p})$ & -393608.4547 & -394132.649 \\
\hline $\mathrm{G} 3(298.15 \mathrm{~K})$ & -393990.5772 \\
$\mathrm{G} 3(0 \mathrm{~K})$ & -393993.9011 \\
\hline
\end{tabular}

TOTAL MULLIKEN AND LOWDIN ATOMIC POPULATIONS

\begin{tabular}{|c|c|c|c|c|}
\hline ATOM & MULL . POP. & CHARGE & LOW. POP. & CHARGE \\
\hline $1 \mathrm{C}$ & 6.357429 & -0.357429 & 6.285911 & -0.285911 \\
\hline $\mathrm{C}$ & 6.082414 & -0.082414 & 6.103256 & -0.103256 \\
\hline $\mathrm{C}$ & 6.082406 & -0.082406 & 6.103250 & -0.103250 \\
\hline $\mathrm{C}$ & 6.357437 & -0.357437 & 6.285933 & -0.285933 \\
\hline $\mathrm{H}$ & 0.827821 & 0.172179 & 0.859207 & 0.140793 \\
\hline $\mathrm{H}$ & 0.851815 & 0.148185 & 0.873176 & 0.126824 \\
\hline $\mathrm{H}$ & 0.851814 & 0.148186 & 0.873175 & 0.126825 \\
\hline $\mathrm{H}$ & 0.827814 & 0.172186 & 0.859207 & 0.140793 \\
\hline S & 15.159079 & 0.840921 & 15.10 & 93857 \\
\hline 10 & 8.6 & -0.601970 & 8.65 & -0.6 \\
\hline
\end{tabular}


benzothiophene-S-oxide (8)

$\begin{array}{lrrrr}\text { MP2/6-31G }(\mathrm{d}, \mathrm{p}) & & & \\ \mathrm{C} & 6.0 & -0.0121352322 & -0.2524124049 & -0.7179325641 \\ \mathrm{C} & 6.0 & -0.4713041518 & 0.2335345925 & 0.5166151294 \\ \mathrm{C} & 6.0 & -1.8885107473 & -0.0575218614 & 0.7124679209 \\ \mathrm{C} & 6.0 & -2.4427100060 & -0.7340048824 & -0.3166447385 \\ \mathrm{C} & 6.0 & 1.3177932992 & -0.1498637359 & -1.1055111987 \\ \mathrm{H} & 1.0 & -2.4309352269 & 0.2391227183 & 1.6016207993 \\ \mathrm{H} & 1.0 & -3.4672086177 & -1.0609679868 & -0.4124252167 \\ \mathrm{~S} & 16.0 & -1.3543365550 & -0.8809499419 & -1.7279219710 \\ \mathrm{O} & 8.0 & -1.1284815007 & -2.3019989712 & -2.1937962269 \\ \mathrm{C} & 6.0 & 2.2136857659 & 0.4512046539 & -0.2167549879 \\ \mathrm{H} & 1.0 & 1.6521919568 & -0.5459715851 & -2.0563728576 \\ \mathrm{H} & 1.0 & 3.2612874103 & 0.5249297885 & -0.4789201506 \\ \mathrm{C} & 6.0 & 1.7667216757 & 0.9664398209 & 1.0069821583 \\ \mathrm{C} & 6.0 & 0.4258382918 & 0.8660594742 & 1.3825516333 \\ \mathrm{H} & 1.0 & 2.4745806990 & 1.4405865169 & 1.6748869701 \\ \mathrm{H} & 1.0 & 0.0870267788 & 1.2645081624 & 2.3314503157\end{array}$

\begin{tabular}{|c|c|c|c|c|}
\hline $\begin{array}{l}\mathrm{MP} 2 / 6- \\
\mathrm{ZPE} \mathrm{Cc} \\
298.15\end{array}$ & $\begin{array}{l}-31 \mathrm{G}(\mathrm{d}, \mathrm{p}) \text { Ener } \\
\text { prrection } \\
\text { 5K Temperature }\end{array}$ & e Correction & $\begin{array}{r}-489519.58 \\
74.04 \\
79.65\end{array}$ & $\begin{array}{ll}351 & \mathrm{KCAL} / \mathrm{MC} \\
4 & \mathrm{KCAL} / \mathrm{MC} \\
5 & \mathrm{KCAL} / \mathrm{MC}\end{array}$ \\
\hline P2/6- & $-311 G(3 d f, 2 p)$ & Energy & -489828.91 & $\mathrm{KCAL}$ \\
\hline OTAL & MULLIKEN AND & LOWDIN ATOMIC & POPULATION & \\
\hline $\mathrm{TOM}$ & MULL. POP. & CHARGE & LOW. POP. & CHARGE \\
\hline & 6.265309 & -0.265309 & 6.17 & -0.17794 \\
\hline $2 \mathrm{C}$ & 5.958366 & 0.041634 & 6. & -0.01 \\
\hline 3 & 6.052741 & -0.052741 & 6. & -0.0 \\
\hline & 6.378284 & -0.378284 & 6 . & -0 \\
\hline C & 6.109718 & -0.1097 & & -0 \\
\hline $\mathrm{H}$ & 0.852180 & 0.14 & & 0.1 \\
\hline $\mathrm{H}$ & 0.833773 & 0.16 & 0 . & 0.1 \\
\hline $\mathrm{S}$ & 15.143139 & 0.856861 & 15 . & 0.8 \\
\hline 0 & 8.612216 & -0.612216 & & -0.65 \\
\hline $\mathrm{C}$ & 1235 & -0.11 & & -0 \\
\hline & 261 & 0.15 & & 0.1 \\
\hline $\mathrm{H}$ & 485 & 0.13 & & 0.1 \\
\hline & 903 & -0.1259 & & -0.1 \\
\hline & 6.1 & -0.1114 & & -0.1 \\
\hline $\mathrm{H}$ & $0 . \varepsilon$ & 0.13 & & 0.1 \\
\hline & 6537 & 0.133463 & 0 . & 0.11 \\
\hline
\end{tabular}


$\operatorname{MP} 2 / 6-31 G(d, p)$

$\begin{array}{rrrrr}\mathrm{C} & 6.0 & -.9443662031 & 1.2552396225 & .2249483537 \\ \mathrm{C} & 6.0 & .3498345325 & .7327061757 & .0735135941 \\ \mathrm{C} & 6.0 & .3497983435 & -.7327014579 & .0734760219 \\ \mathrm{C} & 6.0 & -.9443808146 & -1.2552445574 & .2249474254 \\ \mathrm{C} & 6.0 & -1.1982273792 & 2.6219112468 & .1926438245 \\ \mathrm{~S} & 16.0 & -2.1943213982 & .0001312673 & .5535228580 \\ \mathrm{O} & 8.0 & -3.2991097165 & -.0000629056 & -.4792971119 \\ \mathrm{C} & 6.0 & -.1195988094 & 3.4884726638 & -.0013672529 \\ \mathrm{H} & 1.0 & -2.2080239632 & 2.9998798764 & . .2968487342 \\ \mathrm{H} & 1.0 & -.2887362103 & 4.5565976324 & -.0476654203 \\ \mathrm{C} & 6.0 & 1.1809384704 & 2.9841854042 & -.1273157889 \\ \mathrm{C} & 6.0 & 1.4255482435 & 1.6103793138 & -.0912871369 \\ \mathrm{H} & 1.0 & 2.0080986085 & 3.6692358417 & -.2639771030 \\ \mathrm{H} & 1.0 & 2.4356059602 & 1.2333073369 & -.1986898782 \\ \mathrm{C} & 6.0 & 1.4255518244 & -1.6103915094 & -.0912545541 \\ \mathrm{C} & 6.0 & 1.1809326029 & -2.9841840998 & -.1273717850 \\ \mathrm{H} & 1.0 & 2.4356354110 & -1.2333298819 & -.1987248278 \\ \mathrm{H} & 1.0 & 2.0080910487 & -3.6692365005 & -.2640195513 \\ \mathrm{C} & 6.0 & -.1195808181 & -3.4884895792 & -.0013275390 \\ \mathrm{C} & 6.0 & -1.1982105648 & -2.6219147547 & .1926552121 \\ \mathrm{H} & 1.0 & -.2887321013 & -4.5565940725 & -.0476794105 \\ \mathrm{H} & 1.0 & -2.2080166429 & -2.9998970648 & .2968918367\end{array}$

MP2 / 6-31G (d,p) Energy

ZPE Correction

298.15K Temperature Correction

MP2 / 6-311G (3df,2p) Energy

$$
\begin{array}{cc}
-585649.5602 & \mathrm{KCAL} / \mathrm{MOL} \\
103.2 & \mathrm{KCAL} / \mathrm{MOL} \\
110.77 & \mathrm{KCAL} / \mathrm{MOL} \\
& \\
-586050.8578 & \mathrm{KCAL} / \mathrm{MOL}
\end{array}
$$




$\begin{array}{rrrrrr}\text { TOTAL } & \text { MULLIKEN AND } & \text { LOWDIN ATOMIC } & \text { POPULATIONS } & \\ \text { ATOM } & \text { MULL.POP } & \text { CHARGE } & \text { LOW.POP. } & \text { CHARGE } \\ 1 & \mathrm{C} & 6.270628 & -.270628 & 6.177588 & -.177588 \\ 2 & \mathrm{C} & 5.950831 & .049169 & 6.009762 & -.009762 \\ 3 & \mathrm{C} & 5.950834 & .049166 & 6.009773 & -.009773 \\ 4 & \mathrm{C} & 6.270601 & -.270601 & 6.177541 & -.177541 \\ 5 & \mathrm{C} & 6.123762 & -.123762 & 6.094666 & -.094666 \\ 6 & \mathrm{~S} & 15.125761 & .874239 & 15.133286 & .866714 \\ 7 & \mathrm{O} & 8.613307 & -.613307 & 8.657246 & -.657246 \\ 8 & \mathrm{C} & 6.105718 & -.105718 & 6.096662 & -.096662 \\ 9 & \mathrm{H} & .847659 & .152341 & .873346 & .126654 \\ 10 & \mathrm{H} & .866104 & .133896 & .882784 & .117216 \\ 11 & \mathrm{C} & 6.132696 & -.132696 & 6.102838 & -.102838 \\ 12 & \mathrm{C} & 6.096638 & -.096638 & 6.102512 & -.102512 \\ 13 & \mathrm{H} & .868018 & .131982 & .880739 & .119261 \\ 14 & \mathrm{H} & .868400 & .131600 & .883847 & .116153 \\ 15 & \mathrm{C} & 6.096636 & -.096636 & 6.102517 & -.102517 \\ 16 & \mathrm{C} & 6.132697 & -.132697 & 6.102843 & -.102843 \\ 17 & \mathrm{H} & .868404 & .131596 & .883850 & .116150 \\ 18 & \mathrm{H} & .868023 & .131977 & .880742 & .119258 \\ 19 & \mathrm{C} & 6.105711 & -.105711 & 6.096663 & -.096663 \\ 20 & \mathrm{C} & 6.123803 & -.123803 & 6.094665 & -.094665 \\ 21 & \mathrm{H} & .866103 & .133897 & .882783 & .117217 \\ 22 & \mathrm{H} & .847664 & .152336 & .873347 & .126653\end{array}$

\section{dimethyl sulfilimine (10)}

\begin{tabular}{|c|c|c|c|c|}
\hline $\mathrm{N}$ & 7.0 & 1.7288880220 & -.2919610533 & -1.0713234126 \\
\hline S & 16.0 & .1824994865 & .0147905844 & -.9508987770 \\
\hline $\mathrm{C}$ & 6.0 & -.1592200189 & 1.3961021568 & .1897478006 \\
\hline $\mathrm{C}$ & 6.0 & -.6858755952 & -1.2373966359 & .0497964562 \\
\hline $\mathrm{H}$ & 1.0 & .2505097655 & 1.1821254882 & 1.1768491044 \\
\hline $\mathrm{H}$ & 1.0 & -1.2324358937 & 1.5678083643 & .2608170735 \\
\hline $\mathrm{H}$ & 1.0 & .3292530584 & 2.2743071188 & -.2244291854 \\
\hline $\mathrm{H}$ & 1.0 & -.2381511957 & -1.3023321991 & 1.0414270714 \\
\hline $\mathrm{H}$ & 1.0 & -.5687007526 & -2.1875302714 & -.464797441 \\
\hline $\mathrm{H}$ & 1.0 & -1.7427886690 & -.9880639430 & .1333409668 \\
\hline $\mathrm{H}$ & 1.0 & 2.1360216338 & -.4278489759 & -.1405314036 \\
\hline & $\begin{array}{l}G(d, p) \\
\text { ection } \\
\text { Temper }\end{array}$ & $\begin{array}{l}\text { Energy } \\
\text { ature Correcti }\end{array}$ & $\begin{aligned}-334017.6321 \\
58.77 \\
62.968\end{aligned}$ & $\begin{array}{ll}321 & \mathrm{KCAL} / \mathrm{MOL} \\
7 & \mathrm{KCAL} / \mathrm{MOL} \\
68 & \mathrm{KCAL} / \mathrm{MOL}\end{array}$ \\
\hline
\end{tabular}




\begin{tabular}{lcc}
\hline Basis Set & MP2 $(\mathrm{kcal} / \mathrm{mol})$ & B3LYP $(\mathrm{kcal} / \mathrm{mol})$ \\
\hline $6-31 \mathrm{G}(\mathrm{d})$ & -333980.9405 & -334544.744 \\
$6-31 \mathrm{G}(2 \mathrm{~d})$ & -334016.9821 & -334549.2482 \\
$6-31 \mathrm{G}(\mathrm{d}, \mathrm{p})$ & -334017.6321 & -334552.5362 \\
$6-31+\mathrm{G}(\mathrm{d})$ & -333990.5815 & -334551.4129 \\
$6-31+\mathrm{G}(2 \mathrm{~d}, \mathrm{p})$ & -334062.448 & -334566.1203 \\
$6-311 \mathrm{G}(\mathrm{d})$ & -334040.1773 & -334584.0759 \\
$6-311 \mathrm{G}(3 \mathrm{df}, 2 \mathrm{p})$ & -334170.7417 & -334612.2972 \\
$6-311++\mathrm{G}(\mathrm{d})$ & -334046.1571 & -334587.4031 \\
$6-311++\mathrm{G}(3 \mathrm{df}, 2 \mathrm{p})$ & -334174.5177 & -334615.2992 \\
\hline $\mathrm{G} 3(298.15 \mathrm{~K})$ & -334478.8798 \\
$G 3(0 \mathrm{~K})$ & -334482.6254 \\
\hline
\end{tabular}

TOTAL MULLIKEN AND LOWDIN ATOMIC POPULATIONS

\begin{tabular}{rrrrrr} 
ATOM & MULL.POP. & CHARGE & \multicolumn{1}{c}{ LOW.POP. } & CHARGE \\
1 & $\mathrm{~N}$ & 7.724342 & -.724342 & 7.714447 & -.714447 \\
2 & $\mathrm{~S}$ & 15.325461 & .674539 & 15.248562 & .751438 \\
3 & $\mathrm{C}$ & 6.516525 & -.516525 & 6.461094 & -.461094 \\
4 & $\mathrm{C}$ & 6.516276 & -.516276 & 6.459555 & -.459555 \\
5 & $\mathrm{H}$ & .863111 & .136889 & .886462 & .113538 \\
6 & $\mathrm{H}$ & .863329 & .136671 & .879350 & .120650 \\
7 & $\mathrm{H}$ & .838831 & .161169 & .867449 & .132551 \\
8 & $\mathrm{H}$ & .863035 & .136965 & .886618 & .113382 \\
9 & $\mathrm{H}$ & .838747 & .161253 & .868370 & .131630 \\
10 & $\mathrm{H}$ & .863335 & .136665 & .878824 & .121176 \\
11 & $\mathrm{H}$ & .787008 & .212992 & .849269 & .150731
\end{tabular}

\section{dihydro sulifilimine (11)}
$\operatorname{MP} 2 / 6-31 G(d, p)$

$\begin{array}{rrrrr}\mathrm{N} & 7.0 & -.1115277382 & .3766483045 & -1.0302336294 \\ \mathrm{~S} & 16.0 & .0083515461 & -.3596589277 & .0386544690 \\ \mathrm{H} & 1.0 & 1.1449015298 & .0487091765 & 1.0041225038 \\ \mathrm{H} & 1.0 & -.7139608123 & .03272655967 & 1.3052959500 \\ \mathrm{H} & 1.0 & -.3277644724 & -.3929640429 & -1.6628392404\end{array}$
MP2 / 6-31G (d,p) Energy
ZPE Correction
$\begin{array}{cc}-284827.48320 & \mathrm{KCAL} / \mathrm{MOL} \\ 20.9 & \mathrm{KCAL} / \mathrm{MOL} \\ 23.669 & \mathrm{KCAL} / \mathrm{MOL}\end{array}$ 


\begin{tabular}{lcc}
\hline Basis Set & MP2 $(\mathrm{kcal} / \mathrm{mol})$ & B3LYP $(\mathrm{kcal} / \mathrm{mol})$ \\
\hline $6-31 \mathrm{G}(\mathrm{d})$ & -284807.79252 & -285224.42816 \\
$6-31 \mathrm{G}(2 \mathrm{~d})$ & -284834.46561 & -285232.99433 \\
$6-31 \mathrm{G}(\mathrm{d}, \mathrm{p})$ & -284827.48320 & -285229.99065 \\
$6-31+\mathrm{G}(\mathrm{d})$ & -284815.32555 & -285230.13926 \\
$6-31+\mathrm{G}(2 \mathrm{~d}, \mathrm{p})$ & -284860.29857 & -285244.73324 \\
$6-311 \mathrm{G}(\mathrm{d})$ & -284842.84300 & -285251.69729 \\
$6-311 \mathrm{G}(3 \mathrm{df}, 2 \mathrm{p})$ & -284931.46434 & -285277.13809 \\
$6-311++\mathrm{G}(\mathrm{d})$ & -284849.04763 & \\
$6-311++\mathrm{G}(3 \mathrm{df}, 2 \mathrm{p})$ & -284935.69805 & -285280.84256 \\
\hline $\mathrm{G} 3(298.15 \mathrm{~K})$ & -285180.6400 \\
$G 3(0 \mathrm{~K})$ & -285182.8043 \\
\hline
\end{tabular}

TOTAL MULLIKEN AND LOWDIN ATOMIC POPULATIONS

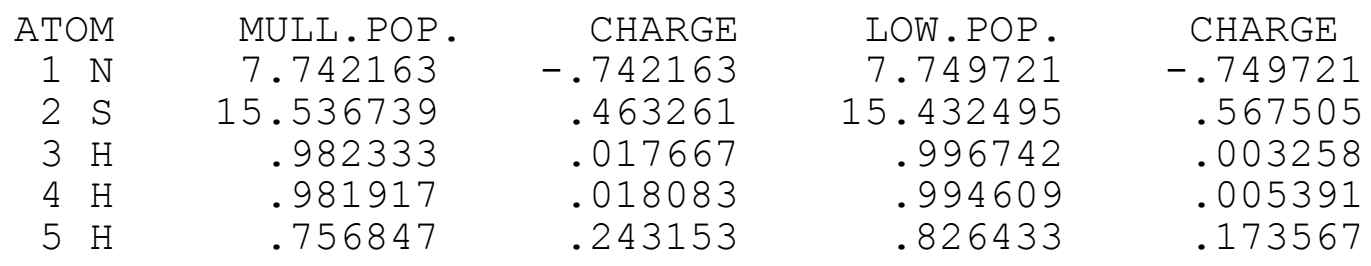

\section{thiirane sulfilimine (12)}

$\operatorname{MP} 2 / 6-31 \mathrm{~g}(\mathrm{~d}, \mathrm{p})$

$\begin{array}{lrrrr}\mathrm{H} & 1.0 & -.9577341266 & .8835459998 & .9382196965 \\ \mathrm{C} & 6.0 & -.0358836914 & .3169308926 & .9790935873 \\ \mathrm{C} & 6.0 & .9422853270 & .4908846569 & -.1226142303 \\ \mathrm{H} & 1.0 & .3292977804 & .0894474166 & 1.9716239753 \\ \mathrm{H} & 1.0 & .6703327541 & 1.1605671840 & -.9268182328 \\ \mathrm{H} & 1.0 & 1.9972440684 & .3695868896 & .0845183357 \\ \mathrm{~S} & 16.0 & -.1112752470 & -1.0791554758 & -.1473166807 \\ \mathrm{~N} & 7.0 & -1.4759792116 & -.8667206626 & -.9458742531 \\ \mathrm{H} & 1.0 & -1.3582877065 & -1.3650869010 & -1.8308320398 \\ & & & \\ \mathrm{MP2/6-31G(d,p)} & \text { Energy } & -333254.75852 \mathrm{KCAL} / \mathrm{MOL} \\ \mathrm{ZPE} \text { Correction } & & 44.49 & \mathrm{KCAL} / \mathrm{MOL} \\ \text { 298.15K Temperature Correction } & 48.083 & \mathrm{KCAL} / \mathrm{MOL}\end{array}$




\begin{tabular}{lcc}
\hline Basis Set & MP2 $(\mathrm{kcal} / \mathrm{mol})$ & B3LYP $(\mathrm{kcal} / \mathrm{mol})$ \\
\hline $6-31 \mathrm{G}(\mathrm{d})$ & -333228.33601 & -333773.26278 \\
$6-31 \mathrm{G}(2 \mathrm{~d})$ & -333262.30041 & -333777.88495 \\
$6-31 \mathrm{G}(\mathrm{d}, \mathrm{p})$ & -333254.75852 & -333779.38134 \\
$6-31+\mathrm{G}(\mathrm{d})$ & -333239.00251 & -333780.82714 \\
$6-31+\mathrm{G}(2 \mathrm{~d}, \mathrm{p})$ & -333297.95176 & -333793.27233 \\
$6-311 \mathrm{G}(\mathrm{d})$ & -333285.41530 & -333812.05892 \\
$6-311 \mathrm{G}(3 \mathrm{df}, 2 \mathrm{p})$ & -333404.27181 & -333838.56503 \\
$6-311++\mathrm{G}(\mathrm{d})$ & -333292.37409 & -333816.32102 \\
$6-311++\mathrm{G}(3 \mathrm{df}, 2 \mathrm{p})$ & -333408.74521 & -333842.12489 \\
\hline G3 (298.15K) & -333716.4640 \\
G3 (0K) & -333719.4986 \\
\hline
\end{tabular}

TOTAL MULLIKEN AND LOWDIN ATOMIC POPULATIONS

\begin{tabular}{|c|c|c|c|c|}
\hline ATOM & MULL . POP. & CHARGE & LOW . POP. & CHARGE \\
\hline $1 \mathrm{H}$ & .804006 & .195994 & .854839 & .145161 \\
\hline $\mathrm{C}$ & 6.350615 & -.350615 & 6.289137 & -.289137 \\
\hline $\mathrm{C}$ & 6.393243 & -.393243 & 6.352980 & -.35298 \\
\hline $\mathrm{H}$ & .835360 & .164640 & .869866 & .1301 \\
\hline $\mathrm{H}$ & 9875 & .17 & .86621 & \\
\hline $\mathrm{H}$ & .85 & .14 & & \\
\hline S & 15.41 & & 15.3 & \\
\hline 8 & 7.75 & -.75 & 7.7 & -.7 \\
\hline 9 & 761110 & 23585 & 8286 & \\
\hline
\end{tabular}

\section{vinyl sulfilimine (13)}

$\operatorname{MP} 2 / 6-31 \mathrm{~g}(\mathrm{~d}, \mathrm{p})$

$\begin{array}{lrrrr}\mathrm{N} & 7.0 & -2.0016227080 & -1.1098660687 & -.1395847156 \\ \mathrm{~S} & 16.0 & -1.0117366705 & -.0255439483 & -.7302358598 \\ \mathrm{C} & 6.0 & .4386587767 & .0889013829 & .2859858052 \\ \mathrm{H} & 1.0 & -1.4349532102 & 1.2665855309 & -.5478451636 \\ \mathrm{C} & 6.0 & 1.6560778955 & .0187232280 & -.1950859628 \\ \mathrm{H} & 1.0 & .2771250799 & .6362242096 & 1.3001793938 \\ \mathrm{H} & 1.0 & 2.5439639961 & .1668990541 & .4035854198 \\ \mathrm{H} & 1.0 & 1.7897919467 & -.3513036451 & -1.2029979932 \\ \mathrm{H} & 1.0 & -2.2573049481 & -.8906196904 & .8259990752 \\ & & & \\ \mathrm{MP2/6-31G(d,p)} \text { Energy } & -333242.43930 & \mathrm{KCAL} / \mathrm{MOL} \\ \mathrm{ZPE} \text { Correction } & & 43.05 & \mathrm{KCAL} / \mathrm{MOL} \\ \text { 298.15K Temperature Correction } & 46.862 & \mathrm{KCAL} / \mathrm{MOL}\end{array}$




\begin{tabular}{lcc}
\hline Basis Set & MP2 $(\mathrm{kcal} / \mathrm{mol})$ & B3LYP $(\mathrm{kcal} / \mathrm{mol})$ \\
\hline $6-31 \mathrm{G}(\mathrm{d})$ & -333214.20020 & -333763.10257 \\
$6-31 \mathrm{G}(2 \mathrm{~d})$ & -333250.29756 & -333769.88648 \\
$6-31 \mathrm{G}(\mathrm{d}, \mathrm{p})$ & -333242.43930 & -333770.06968 \\
$6-31+\mathrm{G}(\mathrm{d})$ & -333224.73692 & -333770.55144 \\
$6-31+\mathrm{G}(2 \mathrm{~d}, \mathrm{p})$ & -333287.19958 & -333785.87861 \\
$6-311 \mathrm{G}(\mathrm{d})$ & -333270.45144 & -333802.76410 \\
$6-311 G(3 \mathrm{df}, 2 \mathrm{p})$ & -333392.60948 & -333831.77631 \\
$6-311++\mathrm{G}(\mathrm{d})$ & -333276.60454 & -333806.54767 \\
$6-311++\mathrm{G}(3 \mathrm{df}, 2 \mathrm{p})$ & -333396.70755 & -333835.07934 \\
\hline G3 (298.15K) & -333708.8987 \\
G3 (0K) & -333712.1969 \\
\hline
\end{tabular}

TOTAL MULLIKEN AND LOWDIN ATOMIC POPULATIONS

\begin{tabular}{lrrrr} 
ATOM & MULL.POP & \multicolumn{1}{c}{ CHARGE } & \multicolumn{1}{c}{ LOW.POP. } & \multicolumn{1}{c}{ CHARGE } \\
$1 \mathrm{~N}$ & 7.706030 & -0.706030 & 7.709744 & -0.709744 \\
$2 \mathrm{~S}$ & 15.408942 & 0.591058 & 15.316155 & 0.683845 \\
$3 \mathrm{C}$ & 6.300454 & -0.300454 & 6.286759 & -0.286759 \\
$4 \mathrm{H}$ & 1.020588 & -0.020588 & 1.018971 & -0.018971 \\
$5 \mathrm{C}$ & 6.217868 & -0.217868 & 6.192982 & -0.192982 \\
$6 \mathrm{H}$ & 0.852608 & 0.147392 & 0.880595 & 0.119405 \\
$7 \mathrm{H}$ & 0.863656 & 0.136344 & 0.876554 & 0.123446 \\
$8 \mathrm{H}$ & 0.858111 & 0.141889 & 0.877136 & 0.122864 \\
$9 \mathrm{H}$ & 0.771745 & 0.228255 & 0.841103 & 0.158897
\end{tabular}

methane sulfilimine (14)

$\operatorname{MP} 2 / 6-31 G(d, p)$

$\begin{array}{rrrrr}\mathrm{N} & 7.0 & -1.1566389047 & 1.1477263743 & -1.0186427781 \\ \mathrm{~S} & 16.0 & -1.0305456804 & .0269196674 & .0881094715 \\ \mathrm{C} & 6.0 & .6778203464 & -.0012954533 & .6812149550 \\ \mathrm{H} & 1.0 & -1.0453325436 & -1.2585200291 & -.0962189080 \\ \mathrm{H} & 1.0 & -.4755631275 & 1.0006692624 & -1.7672991811 \\ \mathrm{H} & 1.0 & 1.3530149984 & -.0793452474 & -.1534270700 \\ \mathrm{H} & 1.0 & .7075846692 & -1.0457693546 & 1.3694690098 \\ \mathrm{H} & 1.0 & .9696599252 & .07096155743 & 1.1967952949\end{array}$

MP2 / 6-31G (d,p) Energy

ZPE Correction

$-309423.78061 \mathrm{KCAL} / \mathrm{MOL}$

$40.2 \mathrm{KCAL} / \mathrm{MOL}$

298.15K Temperature Correction

$43.474 \mathrm{KCAL} / \mathrm{MOL}$ 


\begin{tabular}{lcc}
\hline Basis Set & MP2 $(\mathrm{kcal} / \mathrm{mol})$ & B3LYP $(\mathrm{kcal} / \mathrm{mol})$ \\
\hline $6-31 \mathrm{G}(\mathrm{d})$ & -309395.58652 & -309886.22734 \\
$6-31 \mathrm{G}(2 \mathrm{~d})$ & -309426.14124 & -309892.40305 \\
$6-31 \mathrm{G}(\mathrm{d}, \mathrm{p})$ & -309423.78061 & -309892.84833 \\
$6-31+\mathrm{G}(\mathrm{d})$ & -309403.49812 & -309891.99673 \\
$6-31+\mathrm{G}(2 \mathrm{~d}, \mathrm{p})$ & -309461.41160 & -309906.43094 \\
$6-311 \mathrm{G}(\mathrm{d})$ & -309443.01444 & -309919.86640 \\
$6-311 \mathrm{G}(3 \mathrm{df}, 2 \mathrm{p})$ & -309551.61688 & -309946.05674 \\
$6-311++\mathrm{G}(\mathrm{d})$ & -309448.40519 & -309923.28808 \\
$6-311++\mathrm{G}(3 \mathrm{df}, 2 \mathrm{p})$ & -309555.22512 & -309949.03704 \\
\hline G3 (298.15K) & -309829.8835 \\
G3 (0K) & -309832.6703 \\
\hline
\end{tabular}

\begin{tabular}{|c|c|c|c|c|}
\hline ATOM & MULL. POP. & CHARGE & LOW. POP. & CHARGE \\
\hline $1 \mathrm{~N}$ & 7.714175 & -.714175 & 7.717452 & -.717452 \\
\hline $\mathrm{S}$ & 15.401457 & .598543 & 15.320003 & .679997 \\
\hline $\mathrm{C}$ & 6.528725 & -.528725 & 6.470410 & -.470410 \\
\hline $\mathrm{H}$ & 1.028728 & -.028728 & 1.026291 & -.026291 \\
\hline $\mathrm{H}$ & .774020 & .225980 & .840624 & .159376 \\
\hline $\mathrm{H}$ & .853637 & .146363 & .881132 & .118868 \\
\hline $7 \mathrm{H}$ & .860545 & .13 & .874118 & .125882 \\
\hline 8 & .838712 & .161288 & .869969 & .130031 \\
\hline
\end{tabular}

\section{methyl vinyl sulfilimine (15)}

\begin{tabular}{|c|c|c|c|c|}
\hline $\mathrm{N}$ & 7.0 & 1.6434275994 & -1.3045775145 & .7836345351 \\
\hline S & 16.0 & .9889139202 & -.0610973695 & .0549538339 \\
\hline C & 6.0 & -.6184703437 & .3823201608 & .7251285416 \\
\hline C & 6.0 & .3349559930 & -.5082895397 & -1.5866235735 \\
\hline $\mathrm{H}$ & 1.0 & 1.1754625144 & -.8683070726 & -2.1755169666 \\
\hline $\mathrm{H}$ & 1.0 & -.1075920416 & .3681270549 & -2.0574356333 \\
\hline $\mathrm{H}$ & 1.0 & -.4095699328 & -1.2988864737 & -1.4941871807 \\
\hline $\mathrm{C}$ & 6.0 & -.8204527706 & 1.5900668468 & 1.2613554704 \\
\hline $\mathrm{H}$ & 1.0 & -1.3765228891 & -.3954504077 & .7370831786 \\
\hline $\mathrm{H}$ & 1.0 & -1.7676479965 & 1.8571745796 & 1.7092947436 \\
\hline $\mathrm{H}$ & 1.0 & -.0398378077 & 2.3391405689 & 1.2603189266 \\
\hline $\mathrm{H}$ & 1.0 & .9973328561 & -2.1001291272 & .7821822464 \\
\hline & $\begin{array}{l}G(d, p) \\
\text { ection } \\
\text { Tempera }\end{array}$ & $\begin{array}{l}\text { Energy } \\
\text { ature Correctio }\end{array}$ & $\begin{array}{c}-357837.01900 \\
61.63 \\
66.451\end{array}$ & $\begin{array}{ll}1900 & \mathrm{KCAL} / \mathrm{MOL} \\
3 & \mathrm{KCAL} / \mathrm{MOL} \\
51 & \mathrm{KCAL} / \mathrm{MOL}\end{array}$ \\
\hline
\end{tabular}




\begin{tabular}{lcc}
\hline Basis Set & MP2 $(\mathrm{kcal} / \mathrm{mol})$ & B3LYP $(\mathrm{kcal} / \mathrm{mol})$ \\
\hline $6-31 \mathrm{G}(\mathrm{d})$ & -357800.29100 & -358422.24108 \\
$6-31 \mathrm{G}(2 \mathrm{~d})$ & -357841.81056 & -358427.40126 \\
$6-31 \mathrm{G}(\mathrm{d}, \mathrm{p})$ & -357837.01900 & -358430.34716 \\
$6-31+\mathrm{G}(\mathrm{d})$ & -357812.34939 & -358430.37547 \\
$6-31+\mathrm{G}(2 \mathrm{~d}, \mathrm{p})$ & -357888.70249 & -358445.96939 \\
$6-311 \mathrm{G}(\mathrm{d})$ & -357868.24996 & -358467.41126 \\
$6-311 \mathrm{G}(3 \mathrm{df}, 2 \mathrm{p})$ & -358012.37191 & -358498.52743 \\
$6-311++\mathrm{G}(\mathrm{d})$ & -357874.98067 & -358471.09433 \\
$6-311++\mathrm{G}(3 \mathrm{df}, 2 \mathrm{p})$ & -358016.55370 & -358501.77208 \\
\hline $\mathrm{G} 3(298.15 \mathrm{~K})$ & -358358.3474 \\
$\mathrm{G} 3(0 \mathrm{~K})$ & -358362.6302 \\
\hline
\end{tabular}

TOTAL MULLIKEN AND LOWDIN ATOMIC POPULATIONS

\begin{tabular}{rrrrrr} 
ATOM & MULL.POP. & \multicolumn{1}{c}{ CHARGE } & \multicolumn{1}{c}{ LOW.POP. } & \multicolumn{1}{l}{ CHARGE } \\
1 & $\mathrm{~N}$ & 7.715492 & -.715492 & 7.708575 & -.708575 \\
2 & $\mathrm{~S}$ & 15.322588 & .677412 & 15.238607 & .761393 \\
3 & $\mathrm{C}$ & 6.283850 & -.283850 & 6.279500 & -.279500 \\
4 & $\mathrm{C}$ & 6.519755 & -.519755 & 6.463473 & -.463473 \\
5 & $\mathrm{H}$ & .840701 & .159299 & .868837 & .131163 \\
6 & $\mathrm{H}$ & .855968 & .144032 & .872745 & .127255 \\
7 & $\mathrm{H}$ & .862955 & .137045 & .884940 & .115060 \\
8 & $\mathrm{C}$ & 6.227022 & -.227022 & 6.201687 & -.201687 \\
9 & $\mathrm{H}$ & .859554 & .140446 & .881835 & .118165 \\
10 & $\mathrm{H}$ & .867185 & .132815 & .878687 & .121313 \\
11 & $\mathrm{H}$ & .860323 & .139677 & .875757 & .124243 \\
12 & $\mathrm{H}$ & .784607 & .215393 & .845358 & .154642
\end{tabular}

\section{thiophene sulfilimine (16)}

$\begin{array}{lrrrr}\text { MP2/6-31g }(\mathrm{d}, \mathrm{p}) & & & \\ \mathrm{C} & 6.0 & -.3014173190 & .2993285001 & -1.1987223869 \\ \mathrm{C} & 6.0 & .6317437681 & 1.1139106873 & -.6507272750 \\ \mathrm{C} & 6.0 & .8000284786 & .9054901737 & .7787017063 \\ \mathrm{C} & 6.0 & -.0088898165 & -.0624110961 & 1.2722702946 \\ \mathrm{H} & 1.0 & -.6409665191 & .2519636395 & -2.2214684218 \\ \mathrm{H} & 1.0 & 1.1929053981 & 1.8520320488 & -1.2069344859 \\ \mathrm{H} & 1.0 & 1.4962327033 & 1.4763614199 & 1.3775045068 \\ \mathrm{H} & 1.0 & -.1074549585 & -.4066854117 & 2.2898078660 \\ \mathrm{~S} & 16.0 & -1.1688399667 & -.6217200895 & .0479437609 \\ \mathrm{~N} & 7.0 & -1.4004265480 & -2.1815676570 & -.1563741018 \\ \mathrm{H} & 1.0 & -.4946191702 & -2.6264262480 & -.3320486661\end{array}$

MP2 / 6-31G (d,p) Energy

ZPE Correction

298.15K Temperature Correction $\begin{array}{cc}-380926.5498 & \mathrm{KCAL} / \mathrm{MOL} \\ 51.72 & \mathrm{KCAL} / \mathrm{MOL} \\ 55.784 & \mathrm{KCAL} / \mathrm{MOL}\end{array}$ 


\begin{tabular}{|c|c|c|c|c|}
\hline $\operatorname{MP} 2 / 6$ & $-311 G(3 d f, 2 p)$ & Energy & -381125 & $77 \mathrm{KCAL} /$ \\
\hline TOTAL & MULLIKEN AND & LOWDIN ATOMIC & POPULATIONS & \\
\hline ATOM & MULL . POP. & CHARGE & LOW. POP . & CHARGE \\
\hline $1 \mathrm{C}$ & 6.352291 & -.352291 & 6.288549 & -.288549 \\
\hline $2 \mathrm{C}$ & 6.079937 & -.079937 & 066 & -.11 \\
\hline $3 \mathrm{C}$ & 80215 & -.08021 & 6.1 & -.11 \\
\hline $4 \mathrm{C}$ & 6.352093 & -.352093 & 6.28 & -.28 \\
\hline $5 \mathrm{H}$ & .832286 & .16771 & .86 & .13 \\
\hline $6 \mathrm{H}$ & 991 & .14 & & \\
\hline $7 \mathrm{H}$ & 992 & .14 & & \\
\hline $8 \mathrm{H}$ & 457 & .1675 & .8 & \\
\hline $9 \mathrm{~S}$ & 15.30 & .697 & 15.21 & \\
\hline $10 \mathrm{~N}$ & 1847 & -.691 & 7.68 & -.682 \\
\hline $11 \mathrm{H}$ & .766764 & .233236 & .835178 & .1648 \\
\hline
\end{tabular}

\section{benzothiophene sulfilimine (17)}

$\begin{array}{lrr}\text { MP2 } / 6-31 G(d, p) & \\ \text { H } & 1.0 & -.4099330481 \\ \mathrm{C} & 6.0 & -.2771675748 \\ \mathrm{C} & 6.0 & .0814592152 \\ \mathrm{C} & 6.0 & -.2814944323 \\ \mathrm{C} & 6.0 & -.0756269378 \\ \mathrm{C} & 6.0 & .0901100649 \\ \mathrm{C} & 6.0 & -.0904092777 \\ \mathrm{~S} & 16.0 & -.5904707439 \\ \mathrm{H} & 1.0 & -.0517000459 \\ \mathrm{H} & 1.0 & .2338119129 \\ \mathrm{C} & 6.0 & -.0724674619 \\ \mathrm{H} & 1.0 & .2130289193 \\ \mathrm{H} & 1.0 & .0605359668 \\ \mathrm{C} & 6.0 & -.2305166476 \\ \mathrm{~N} & 7.0 & . .3388023469 \\ \mathrm{H} & 1.0 & -.2583507562 \\ \mathrm{H} & 1.0 & 1.3203877594\end{array}$

MP2/6-31G (d,p) Energy

ZPE Correction

298.15K Temperature Correction

MP2 / 6-311G (3df,2p) Energy

$$
\begin{array}{rr}
1.1032105089 & -2.3432218414 \\
1.1021145774 & -1.2680334524 \\
1.0604943318 & 1.5405817419 \\
-.0905383635 & -.5555174048 \\
2.2878107399 & -.5564861333 \\
2.2647832154 & .8353330342 \\
-.1345358891 & .8333984349 \\
-1.7234100015 & -1.2530845244 \\
3.2333943315 & -1.0829781559 \\
3.1954348306 & 1.3693636284 \\
-1.5065022331 & 1.3319569083 \\
1.0494427172 & 2.6162000132 \\
-1.7451528079 & 2.3800502507 \\
-2.4312369908 & .3612109671 \\
-2.1486733658 & -2.4690074283 \\
-3.5056693569 & .4685532252 \\
-2.0109660331 & -2.2083195784
\end{array}
$$

$$
\begin{array}{cc}
-477055.4315 & \mathrm{KCAL} / \mathrm{MOL} \\
81.25 & \mathrm{KCAL} / \mathrm{MOL} \\
87.024 & \mathrm{KCAL} / \mathrm{MOL}
\end{array}
$$

$-477346.297 \mathrm{KCAL} / \mathrm{MOL}$ 


\begin{tabular}{rrrrrr}
\multicolumn{2}{l}{ TOTAL } & MULLIKEN AND & LOWDIN ATOMIC & POPULATIONS & \\
ATOM & MULL.POP & CHARGE & LOW.POP. & \multicolumn{1}{l}{ CHARGE } \\
$1 \mathrm{H}$ & .847186 & .152814 & .874512 & .125488 \\
2 & $\mathrm{C}$ & 6.114182 & -.114182 & 6.094837 & -.094837 \\
3 & $\mathrm{C}$ & 6.113010 & -.113010 & 6.103268 & -.103268 \\
4 & $\mathrm{C}$ & 6.265546 & -.265546 & 6.183553 & -.183553 \\
5 & $\mathrm{C}$ & 6.112049 & -.112049 & 6.101893 & -.101893 \\
6 & $\mathrm{C}$ & 6.126700 & -.126700 & 6.105257 & -.105257 \\
7 & $\mathrm{C}$ & 5.955316 & .044684 & 6.019654 & -.019654 \\
8 & $\mathrm{~S}$ & 15.275337 & .724663 & 15.227001 & .772999 \\
9 & $\mathrm{H}$ & .867240 & .132760 & .881098 & .118902 \\
10 & $\mathrm{H}$ & .869166 & .130834 & .882041 & .117959 \\
11 & $\mathrm{C}$ & 6.052914 & -.052914 & 6.096141 & -.096141 \\
12 & $\mathrm{H}$ & .868633 & .131367 & .885108 & .114892 \\
13 & $\mathrm{H}$ & .855227 & .144773 & .877493 & .122507 \\
14 & $\mathrm{C}$ & 6.373604 & -.373604 & 6.282550 & -.282550 \\
15 & $\mathrm{~N}$ & 7.696034 & -.696034 & 7.682385 & -.682385 \\
16 & $\mathrm{H}$ & .838039 & .161961 & .865224 & .134776 \\
17 & $\mathrm{H}$ & .769817 & .230183 & .837983 & .162017
\end{tabular}

dibenzothiophene sulfilimine (18)

$\operatorname{MP} 2 / 6-31 G(d, p)$

$\begin{array}{rrrrr}\mathrm{H} & 1.0 & 0.5050339476 & -3.5909463896 & -0.4610351059 \\ \mathrm{C} & 6.0 & 0.4988955948 & -2.7814020230 & 0.2590616450 \\ \mathrm{C} & 6.0 & 0.4862963372 & -0.6396139296 & 2.1034182784 \\ \mathrm{C} & 6.0 & 0.0853855527 & -1.5079731143 & -0.1155717633 \\ \mathrm{C} & 6.0 & 0.9186153467 & -2.9767357658 & 1.5769468331 \\ \mathrm{C} & 6.0 & 0.9015292479 & -1.9145360029 & 2.4906144583 \\ \mathrm{C} & 6.0 & 0.0837810926 & -0.4283128704 & 0.7802030333 \\ \mathrm{~S} & 16.0 & -0.5410681213 & -1.0925221631 & -1.7649737619 \\ \mathrm{H} & 1.0 & 1.2543637732 & -3.9550889429 & 1.8966729202 \\ \mathrm{H} & 1.0 & 1.2223509755 & -2.0837687789 & 3.5106323235 \\ \mathrm{C} & 6.0 & -0.3287373153 & 0.8203926575 & 0.1333709275 \\ \mathrm{H} & 1.0 & 0.4865925698 & 0.1744942656 & 2.8186976128 \\ \mathrm{C} & 6.0 & -1.0434077545 & 2.9728709805 & -1.5038693373 \\ \mathrm{C} & 6.0 & -0.6222708861 & 0.6358895176 & -1.2259725397 \\ \mathrm{C} & 6.0 & -0.4209991322 & 2.1036215033 & 0.6830092688 \\ \mathrm{C} & 6.0 & -0.7775791087 & 3.1710454668 & -0.1423866078 \\ \mathrm{C} & 6.0 & -0.9756817672 & 1.6923221983 & -2.0578447255 \\ \mathrm{H} & 1.0 & -0.2094377817 & 2.2719433691 & 1.7324164637 \\ \mathrm{H} & 1.0 & -0.8426781731 & 4.1682982978 & 0.2740223944 \\ \mathrm{H} & 1.0 & -1.1783004469 & 1.5201797513 & -3.1082650016 \\ \mathrm{H} & 1.0 & -1.3073715905 & 3.8169584911 & -2.1282504477 \\ \mathrm{~N} & 7.0 & 0.4422837669 & -1.3924438033 & -2.9734215884 \\ \mathrm{H} & 1.0 & 1.3624034486 & -0.9846729789 & -2.7774746978\end{array}$

MP2 / 6-31G (d,p) Energy

ZPE Correction

$-573185.4619 \mathrm{KCAL} / \mathrm{MOI}$

$110.5 \mathrm{KCAL} / \mathrm{MOL}$

$118.192 \mathrm{KCAL} / \mathrm{MOL}$

298.15K Temperature Correction

MP2/6-311G (3df,2p) Energy

$-573568.2915 \mathrm{KCAL} / \mathrm{MOL}$ 


\begin{tabular}{rrrrrr}
\multicolumn{1}{l}{ TOTAL } & MULLIKEN AND & LOWDIN ATOMIC & \multicolumn{1}{l}{ POPULATIONS } & \\
ATOM & MULL.POP. & CHARGE & \multicolumn{1}{l}{ LOW.POP. } & \multicolumn{1}{c}{ CHARGE } \\
1 & $\mathrm{H}$ & 0.848056 & 0.151944 & 0.871321 & 0.128679 \\
2 & $\mathrm{C}$ & 6.128803 & -0.128803 & 6.101119 & -0.101119 \\
3 & $\mathrm{C}$ & 6.098288 & -0.098288 & 6.106066 & -0.106066 \\
4 & $\mathrm{C}$ & 6.269130 & -0.269130 & 6.181942 & -0.181942 \\
5 & $\mathrm{C}$ & 6.106459 & -0.106459 & 6.100230 & -0.100230 \\
6 & $\mathrm{C}$ & 6.133446 & -0.133446 & 6.102341 & -0.102341 \\
7 & $\mathrm{C}$ & 5.950428 & 0.049572 & 6.013581 & -0.013581 \\
8 & $\mathrm{~S}$ & 15.250377 & 0.749623 & 15.230917 & 0.769083 \\
9 & $\mathrm{H}$ & 0.867569 & 0.132431 & 0.881711 & 0.118289 \\
10 & $\mathrm{H}$ & 0.869563 & 0.130437 & 0.884030 & 0.115970 \\
11 & $\mathrm{C}$ & 5.950418 & 0.049582 & 6.014524 & -0.014524 \\
12 & $\mathrm{H}$ & 0.869839 & 0.130161 & 0.882604 & 0.117396 \\
13 & $\mathrm{C}$ & 6.106498 & -0.106498 & 6.102122 & -0.102122 \\
14 & $\mathrm{C}$ & 6.269165 & -0.269165 & 6.182995 & -0.182995 \\
15 & $\mathrm{C}$ & 6.098288 & -0.098288 & 6.102267 & -0.102267 \\
16 & $\mathrm{C}$ & 6.133431 & -0.133431 & 6.103271 & -0.103271 \\
17 & $\mathrm{C}$ & 6.128765 & -0.128765 & 6.097541 & -0.097541 \\
18 & $\mathrm{H}$ & 0.869858 & 0.130142 & 0.885604 & 0.114396 \\
19 & $\mathrm{H}$ & 0.869506 & 0.130494 & 0.883369 & 0.116631 \\
20 & $\mathrm{H}$ & 0.848009 & 0.151991 & 0.874261 & 0.125739 \\
21 & $\mathrm{H}$ & 0.867579 & 0.132421 & 0.880049 & 0.119951 \\
22 & $\mathrm{~N}$ & 7.696631 & -0.696631 & 7.680936 & -0.680936 \\
23 & $\mathrm{H}$ & 0.769894 & 0.230106 & 0.837200 & 0.162800
\end{tabular}

\section{dimethyl S,C-methylide (19)}

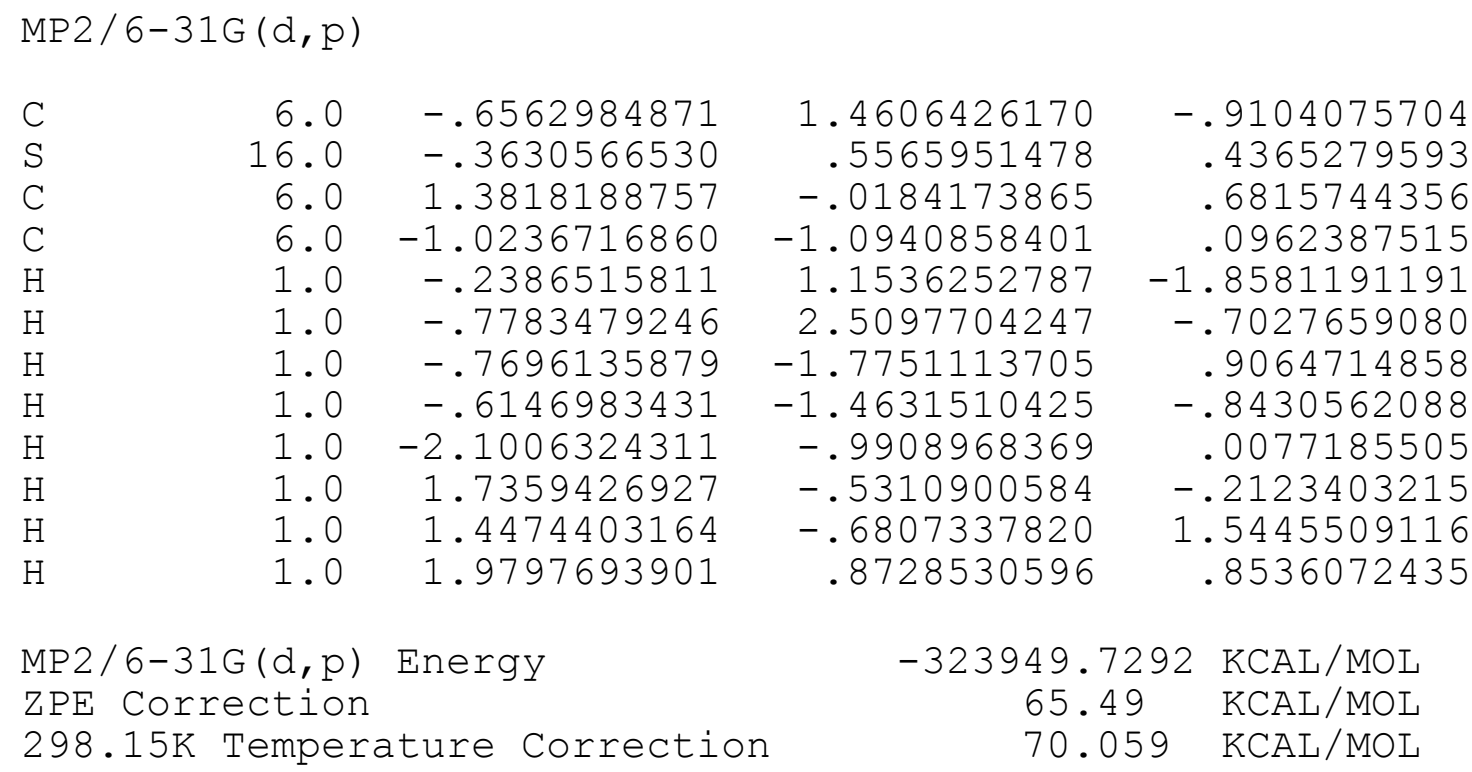




\begin{tabular}{lcc}
\hline Basis Set & MP2 (kcal/mol) & B3LYP $(\mathrm{kcal} / \mathrm{mol})$ \\
\hline $6-31 G(\mathrm{~d})$ & -323908.8677 & -324467.5814 \\
$6-31 G(2 \mathrm{~d})$ & -323940.6027 & -324469.3150 \\
$6-31 \mathrm{G}(\mathrm{d}, \mathrm{p})$ & -323949.7292 & -324475.2630 \\
$6-31+\mathrm{G}(\mathrm{d})$ & -323917.7242 & -324473.7618 \\
$6-31+\mathrm{G}(2 \mathrm{~d}, \mathrm{p})$ & -323989.5210 & -324485.8678 \\
$6-311 \mathrm{G}(\mathrm{d})$ & -323966.9474 & -324504.5792 \\
$6-311 G(3 \mathrm{df}, 2 \mathrm{p})$ & -324088.8786 & -324527.8981 \\
$6-311++\mathrm{G}(\mathrm{d})$ & -323971.2012 & -324506.4519 \\
$6-311++\mathrm{G}(3 \mathrm{df}, 2 \mathrm{p})$ & -324091.3596 & -324529.6722 \\
\hline G3 (298.15K) & -324393.2159 \\
G3 (0K) & -324397.3274 \\
\hline
\end{tabular}

TOTAL MULLIKEN AND LOWDIN ATOMIC POPULATIONS

\begin{tabular}{|c|c|c|c|c|}
\hline ATOM & MULL . POP . & CHARGE & LOW . POP . & CHARGE \\
\hline $1 \mathrm{C}$ & 6.560064 & -.560064 & 6.689490 & -.689490 \\
\hline $2 \mathrm{~s}$ & 15.530696 & .469304 & 15.348773 & .651227 \\
\hline $3 \mathrm{C}$ & 6.494033 & -.494033 & 6.471655 & -.471655 \\
\hline $\mathrm{C}$ & 6.468453 & -.468453 & 6.431169 & -.431169 \\
\hline $\mathrm{H}$ & .907274 & .092726 & .900714 & .099286 \\
\hline $\mathrm{H}$ & .883086 & .116914 & .881759 & .118241 \\
\hline $\mathrm{H}$ & .868985 & .131015 & .880726 & .119274 \\
\hline $\mathrm{H}$ & .854865 & .145135 & .881378 & .118622 \\
\hline $\mathrm{H}$ & 128 & 0872 & 9808 & .130192 \\
\hline 10 & 631 & 369 & 6593 & .113407 \\
\hline 11 & 00 & 8200 & .882571 & .117429 \\
\hline $12 \mathrm{H}$ & & 015 & 5363 & \\
\hline
\end{tabular}

\section{dihydro $S, C$-methylide (20)}

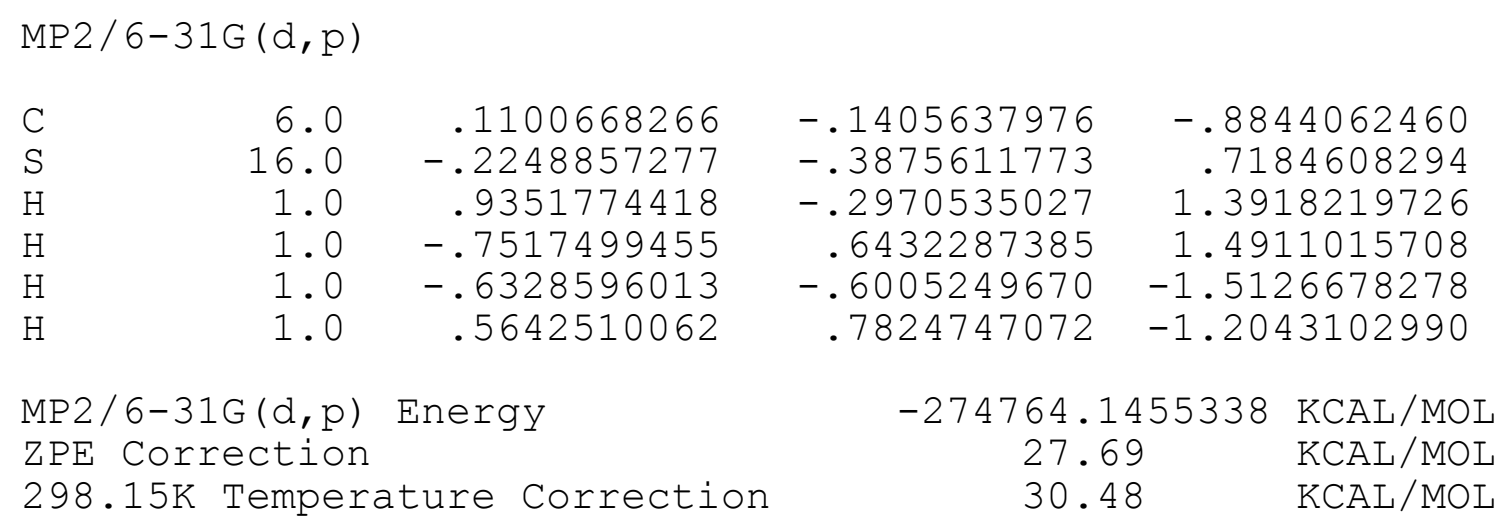




\begin{tabular}{lcc}
\hline Basis Set & MP2 (kcal/mol) & B3LYP $(\mathrm{kcal} / \mathrm{mol})$ \\
\hline $6-31 G(\mathrm{~d})$ & -274740.5236467 & -275152.5614458 \\
$6-31 \mathrm{G}(\mathrm{d}, \mathrm{P})$ & -274764.1455338 & -275157.8725817 \\
$6-31+\mathrm{G}(\mathrm{d})$ & -274746.0567227 & -275156.7363445 \\
$6-311 \mathrm{G}(\mathrm{d})$ & -274775.0109740 & -275178.3211522 \\
$6-311 \mathrm{G}(3 \mathrm{df}, 2 \mathrm{p})$ & -274853.0370655 & -275197.0891772 \\
$6-311++\mathrm{G}(3 \mathrm{df}, 2 \mathrm{p})$ & -274854.9772404 & -275198.6525371 \\
\hline G3 (298.15K) & -275098.1306 \\
G3 (0K) & -275100.4442 \\
\hline
\end{tabular}

\begin{tabular}{rrrrrr} 
TOTAL & MULLIKEN AND & LOWDIN ATOMIC & \multicolumn{1}{l}{ POPULATIONS } & \\
ATOM & MULL.POP. & CHARGE & LOW.POP. & \multicolumn{1}{c}{ CHARGE } \\
$1 \mathrm{C}$ & 6.556184 & -.556184 & 6.682328 & -.682328 \\
$2 \mathrm{~S}$ & 15.703424 & .296576 & 15.531441 & .468559 \\
$3 \mathrm{H}$ & .943462 & .056538 & .973835 & .026165 \\
4 & $\mathrm{H}$ & 1.034717 & -.034717 & 1.041625 & -.041625 \\
$5 \mathrm{H}$ & .874543 & .125457 & .877755 & .122245 \\
$6 \mathrm{H}$ & .887670 & .112330 & .893015 & .106985
\end{tabular}

vinyl $S, C$-methylide (21)

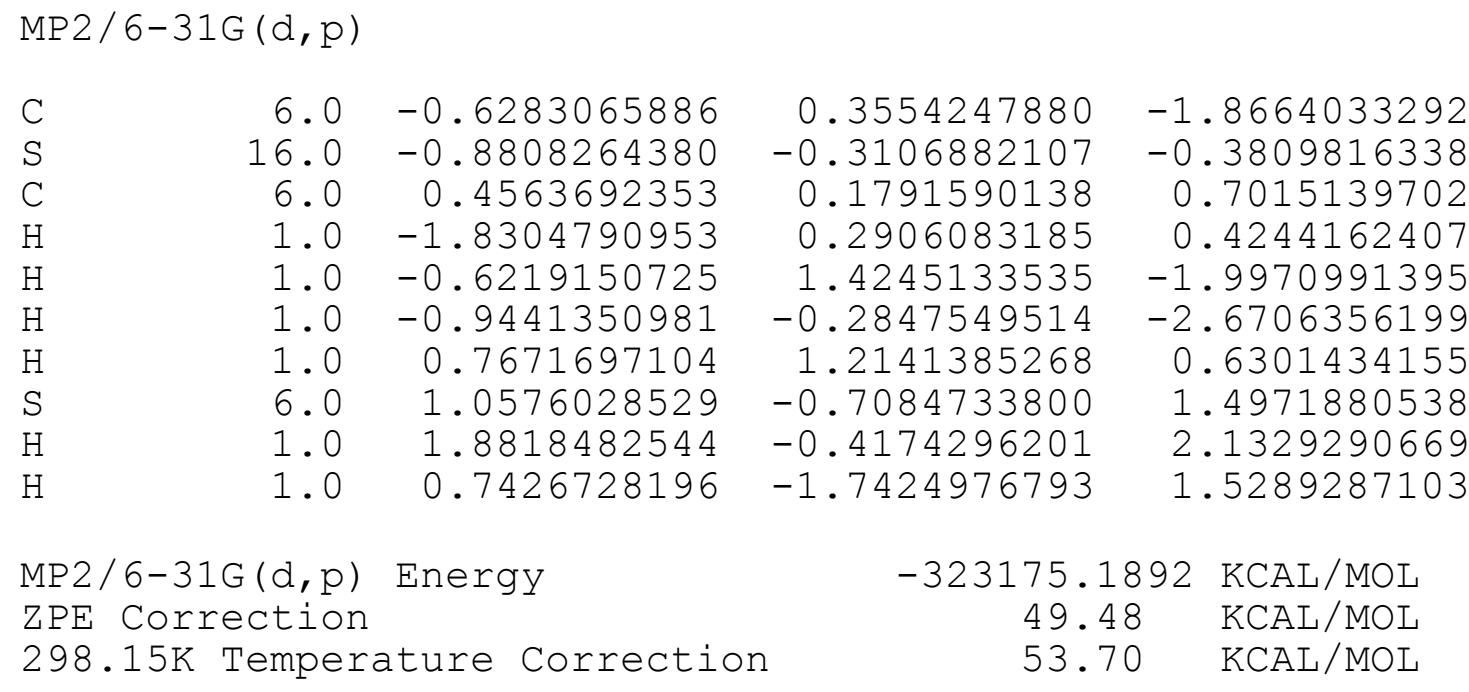




\begin{tabular}{lcc}
\hline Basis Set & MP2 (kcal/mol) & B3LYP $(\mathrm{kcal} / \mathrm{mol})$ \\
\hline $6-31 \mathrm{G}(\mathrm{d})$ & -323142.9117 & -323686.5893 \\
$6-31 \mathrm{G}(2 \mathrm{~d})$ & -323174.4426 & -323690.6436 \\
$6-31 \mathrm{G}(\mathrm{d}, \mathrm{p})$ & -323175.1892 & -323693.4038 \\
$6-31+\mathrm{G}(\mathrm{d})$ & -323152.5211 & -323693.4012 \\
$6-311 \mathrm{G}(\mathrm{d})$ & -323197.8313 & -323723.9264 \\
$6-311 \mathrm{G}(3 \mathrm{df}, 2 \mathrm{p})$ & -323311.2642 & -323748.086 \\
$6-311++\mathrm{G}(\mathrm{d})$ & -323202.0424 & -323726.0075 \\
$6-311++\mathrm{G}(3 \mathrm{df}, 2 \mathrm{p})$ & -323314.0228 & -323750.1381 \\
\hline G3 (298.15K) & -323624.2928 \\
G3 (0K) & -323627.9982 \\
\hline
\end{tabular}

TOTAL MULLIKEN AND LOWDIN ATOMIC POPULATIONS

\begin{tabular}{rrrrrr} 
ATOM & MULL.POP. & CHARGE & LOW.POP. & \multicolumn{1}{c}{ CHARGE } \\
1 & $\mathrm{C}$ & 6.564649 & -0.564649 & 6.684863 & -0.684863 \\
2 & $\mathrm{~S}$ & 15.612758 & 0.387242 & 15.430026 & 0.569974 \\
3 & $\mathrm{C}$ & 6.240086 & -0.240086 & 6.252740 & -0.252740 \\
4 & $\mathrm{H}$ & 1.011406 & -0.011406 & 1.022324 & -0.022324 \\
5 & $\mathrm{H}$ & 0.892234 & 0.107766 & 0.896006 & 0.103994 \\
6 & $\mathrm{H}$ & 0.873258 & 0.126742 & 0.874671 & 0.125329 \\
7 & $\mathrm{H}$ & 0.847204 & 0.152796 & 0.876752 & 0.123248 \\
8 & $\mathrm{~S}$ & 6.227644 & -0.227644 & 6.205425 & -0.205425 \\
9 & $\mathrm{H}$ & 0.866569 & 0.133431 & 0.877209 & 0.122791 \\
10 & $\mathrm{H}$ & 0.864194 & 0.135806 & 0.879983 & 0.120017
\end{tabular}

\section{methane $S, C$-methylide (22)}

$\operatorname{MP} 2 / 6-31 G(d, p)$

$\begin{array}{lrrrr}\mathrm{C} & 6.0 & -1.2387177501 & -0.1460988956 & 0.1774452124 \\ \mathrm{~S} & 16.0 & 0.2928310045 & -0.3259645005 & -0.7659029262 \\ \mathrm{C} & 6.0 & 1.5436599571 & -0.1042993134 & 0.2877286165 \\ \mathrm{H} & 1.0 & -0.0686830052 & 0.7241220359 & -1.6019184319 \\ \mathrm{H} & 1.0 & 2.4352085140 & -0.6306331287 & -0.0071715037 \\ \mathrm{H} & 1.0 & 1.6375656027 & 0.8294808339 & 0.8199725462 \\ \mathrm{H} & 1.0 & -1.2053249127 & 0.7683761810 & 0.7644612426 \\ \mathrm{H} & 1.0 & -1.3173840732 & -1.0021220549 & 0.8405540204 \\ \mathrm{H} & 1.0 & -2.0791550190 & -0.1128611577 & -0.5151686693\end{array}$

MP2 / 6-31G (d,p) Energy

ZPE Correction

$$
\begin{array}{cc}
-299357.8883 & \mathrm{KCAL} / \mathrm{MOL} \\
46.86 & \mathrm{KCAL} / \mathrm{MOL} \\
50.372 & \mathrm{KCAL} / \mathrm{MOL}
\end{array}
$$




\begin{tabular}{lcc}
\hline Basis Set & MP2 $(\mathrm{kcal} / \mathrm{mol})$ & B3LYP $(\mathrm{kcal} / \mathrm{mol})$ \\
\hline $6-31 \mathrm{G}(\mathrm{d})$ & -299325.6531 & -299811.2052 \\
$6-31 \mathrm{G}(2 \mathrm{~d})$ & -299351.5459 & -299814.5538 \\
$6-31 \mathrm{G}(\mathrm{d}, \mathrm{p})$ & -299357.8883 & -299817.6413 \\
$6-31+\mathrm{G}(\mathrm{d})$ & -299332.7065 & -299816.3411 \\
$6-311 \mathrm{G}(\mathrm{d})$ & -299371.7425 & -299842.4823 \\
$6-311 \mathrm{G}(3 \mathrm{df}, 2 \mathrm{p})$ & -299471.3475 & -299863.4011 \\
$6-311++\mathrm{G}(\mathrm{d})$ & -299375.3117 & -299844.2565 \\
$6-311++\mathrm{G}(3 \mathrm{df}, 2 \mathrm{p})$ & -299473.5586 & -299865.0698 \\
\hline G3 (298.15K) & -299746.1097 \\
G3 (0K) & -299749.1952 \\
\hline
\end{tabular}

\begin{tabular}{crrrrr} 
TOTAL & MULLIKEN AND & LOWDIN ATOMIC & \multicolumn{1}{l}{ POPULATIONS } & \\
ATOM & MULL.POP. & CHARGE & \multicolumn{1}{c}{ LOW.POP. } & \multicolumn{1}{c}{ CHARGE } \\
1 & $\mathrm{C}$ & 6.474730 & -0.474730 & 6.438236 & -0.438236 \\
2 & $\mathrm{~S}$ & 15.597257 & 0.402743 & 15.434998 & 0.565002 \\
3 & $\mathrm{C}$ & 6.558977 & -0.558977 & 6.684839 & -0.684839 \\
4 & $\mathrm{H}$ & 1.039100 & -0.039100 & 1.040688 & -0.040688 \\
5 & $\mathrm{H}$ & 0.877285 & 0.122715 & 0.877900 & 0.122100 \\
6 & $\mathrm{H}$ & 0.896175 & 0.103825 & 0.896530 & 0.103470 \\
7 & $\mathrm{H}$ & 0.847481 & 0.152519 & 0.877810 & 0.122190 \\
8 & $\mathrm{H}$ & 0.840527 & 0.159473 & 0.869120 & 0.130880 \\
9 & $\mathrm{H}$ & 0.868467 & 0.131533 & 0.879878 & 0.120122
\end{tabular}

methyl vinyl $S, C$-methylide (23)

\begin{tabular}{lrrrr} 
MP2/6-31G $(\mathrm{d}, \mathrm{p})$ & & \\
$\mathrm{C}$ & 6.0 & -0.6283065886 & 0.3554247880 & -1.8664033292 \\
$\mathrm{~S}$ & 16.0 & -0.8808264380 & -0.3106882107 & -0.3809816338 \\
$\mathrm{C}$ & 6.0 & 0.4563692353 & 0.1791590138 & 0.7015139702 \\
$\mathrm{H}$ & 1.0 & -1.8304790953 & 0.2906083185 & 0.4244162407 \\
$\mathrm{H}$ & 1.0 & -0.6219150725 & 1.4245133535 & -1.9970991395 \\
$\mathrm{H}$ & 1.0 & -0.9441350981 & -0.2847549514 & -2.6706356199 \\
$\mathrm{H}$ & 1.0 & 0.7671697104 & 1.2141385268 & 0.6301434155 \\
$\mathrm{~S}$ & 6.0 & 1.0576028529 & -0.7084733800 & 1.4971880538 \\
$\mathrm{H}$ & 1.0 & 1.8818482544 & -0.4174296201 & 2.1329290669 \\
$\mathrm{H}$ & 1.0 & 0.7426728196 & -1.7424976793 & 1.5289287103 \\
\multicolumn{5}{c}{} \\
MP2/6-31G(d,p) & Energy & $-347771.7553 \mathrm{KCAL} / \mathrm{MOL}$ \\
ZPE Correction & & 68.44 & $\mathrm{KCAL} / \mathrm{MOL}$ \\
298.15K Temperature Correction & $73.453 \mathrm{KCAL} / \mathrm{MOL}$
\end{tabular}




\begin{tabular}{lcc}
\hline Basis Set & MP2 $(\mathrm{kcal} / \mathrm{mol})$ & B3LYP $(\mathrm{kcal} / \mathrm{mol})$ \\
\hline $6-31 \mathrm{G}(\mathrm{d})$ & -347730.8485 & -348347.0647 \\
$6-31 \mathrm{G}(2 \mathrm{~d})$ & -347768.3948 & -348349.4485 \\
$6-31 \mathrm{G}(\mathrm{d}, \mathrm{p})$ & -347771.7553 & -348355.1279 \\
$6-31+\mathrm{G}(\mathrm{d})$ & -347741.6146 & -348354.3408 \\
$6-311 \mathrm{G}(\mathrm{d})$ & -347797.5031 & -348389.8556 \\
$6-311 \mathrm{G}(3 \mathrm{df}, 2 \mathrm{p})$ & -347933.4787 & -348416.1332 \\
$6-311++\mathrm{G}(\mathrm{d})$ & -347802.2068 & -348391.8336 \\
$6-311++\mathrm{G}(3 \mathrm{df}, 2 \mathrm{p})$ & -347936.2515 & -348418.073 \\
\hline G3 (298.15K) & -348275.0386 \\
G3 (0K) & -348279.5912 \\
\hline
\end{tabular}

TOTAL MULLIKEN AND LOWDIN ATOMIC POPULATIONS

\begin{tabular}{rrrrrr} 
ATOM & MULL.POP. & CHARGE & \multicolumn{1}{c}{ LOW.POP. } & \multicolumn{1}{c}{ CHARGE } \\
1 & $\mathrm{C}$ & 6.274128 & -0.274128 & 6.287019 & -0.287019 \\
2 & $\mathrm{C}$ & 6.233598 & -0.233598 & 6.221648 & -0.221648 \\
3 & $\mathrm{H}$ & 0.873389 & 0.126611 & 0.880595 & 0.119405 \\
4 & $\mathrm{H}$ & 0.869749 & 0.130251 & 0.879572 & 0.120428 \\
5 & $\mathrm{H}$ & 0.868479 & 0.131521 & 0.879304 & 0.120696 \\
6 & $\mathrm{~S}$ & 15.509626 & 0.490374 & 15.335834 & 0.664166 \\
7 & $\mathrm{C}$ & 6.471667 & -0.471667 & 6.428427 & -0.428427 \\
8 & $\mathrm{C}$ & 6.568975 & -0.568975 & 6.686522 & -0.686522 \\
9 & $\mathrm{H}$ & 0.876025 & 0.123975 & 0.878339 & 0.121661 \\
10 & $\mathrm{H}$ & 0.898198 & 0.101802 & 0.895411 & 0.104589 \\
11 & $\mathrm{H}$ & 0.868037 & 0.131963 & 0.880245 & 0.119755 \\
12 & $\mathrm{H}$ & 0.846863 & 0.153137 & 0.877351 & 0.122649 \\
13 & $\mathrm{H}$ & 0.841267 & 0.158733 & 0.869734 & 0.130266
\end{tabular}

\section{thiophene $S, C$-methylide (24)}

$\operatorname{MP} 2 / 6-31 G(d, p)$

$\begin{array}{lrrrr}\mathrm{C} & 6.0 & -.6069701274 & .9028444423 & .7724252010 \\ \mathrm{C} & 6.0 & -1.1572638994 & 1.1814645112 & -.4340661626 \\ \mathrm{C} & 6.0 & -.5196268920 & .4707682617 & -1.5283341084 \\ \mathrm{C} & 6.0 & .5081788313 & -.03145860042 & -1.1271187171 \\ \mathrm{H} & 1.0 & -.8592755659 & 1.3033693234 & 1.7421547059 \\ \mathrm{H} & 1.0 & -1.9845434712 & 1.8620398596 & -.5829289402 \\ \mathrm{H} & 1.0 & -.8378057921 & .5655003994 & -2.5565908462 \\ \mathrm{H} & 1.0 & 1.1539379786 & -.9529545774 & -1.7080332100 \\ \mathrm{~S} & 16.0 & .8562757656 & -.1185383395 & .5850526911 \\ \mathrm{C} & 6.0 & 1.1405832193 & -1.5517453221 & 1.3608838363 \\ \mathrm{H} & 1.0 & 1.8456722798 & -1.4468403847 & 2.1677133106 \\ \mathrm{H} & 1.0 & .3287347203 & -2.2553953995 & 1.4521724758\end{array}$


$\operatorname{MP} 2 / 6-31 G(d, p)$ Energy

ZPE Correction

298.15K Temperature Correction

MP2 / 6-311G (3df,2p) Energy

TOTAL MULLIKEN AND LOWDIN ATOMIC POPULATIONS

$\begin{array}{rrrr}\text { ATOM } & \text { MULL.POP. } & \text { CHARGE } \\ 1 & \mathrm{C} & 6.328511 & -.328511 \\ 2 & \mathrm{C} & 6.084331 & -.084331 \\ 3 & \mathrm{C} & 6.085460 & -.085460 \\ 4 & \mathrm{C} & 6.311802 & -.311802 \\ 5 & \mathrm{H} & .846252 & .153748 \\ 6 & \mathrm{H} & .859931 & .140069 \\ 7 & \mathrm{H} & .857920 & .142080 \\ 8 & \mathrm{H} & .831500 & .168500 \\ 9 & \mathrm{~S} & 15.489141 & .510859 \\ 10 & \mathrm{C} & 6.538890 & -.538890 \\ 11 & \mathrm{H} & .879453 & .120547 \\ 12 & \mathrm{H} & .886807 & .113193\end{array}$

$-370861.2280 \mathrm{KCAL} / \mathrm{MOL}$

$58.58 \mathrm{KCAL} / \mathrm{MOL}$

$62.906 \mathrm{KCAL} / \mathrm{MOL}$

$-371045.8281 \mathrm{KCAL} / \mathrm{MOL}$

\section{benzothiophene $S, C$-methylide (25)}

LOW.POP. CHARGE

$6.292410-.292410$

$6.115134-.115134$

$6.115403-.115403$

$6.273449-.273449$

$.865838 \quad .134162$

$.877109 \quad .122891$

$.876547 \quad .123453$

$.856719 \quad .143281$

$\begin{array}{ll}15.301787 & .698213\end{array}$

$6.661141-.661141$

$.876778 \quad .123222$

$.887686 \quad .112314$

MP 2 / 6-31G (d,p)

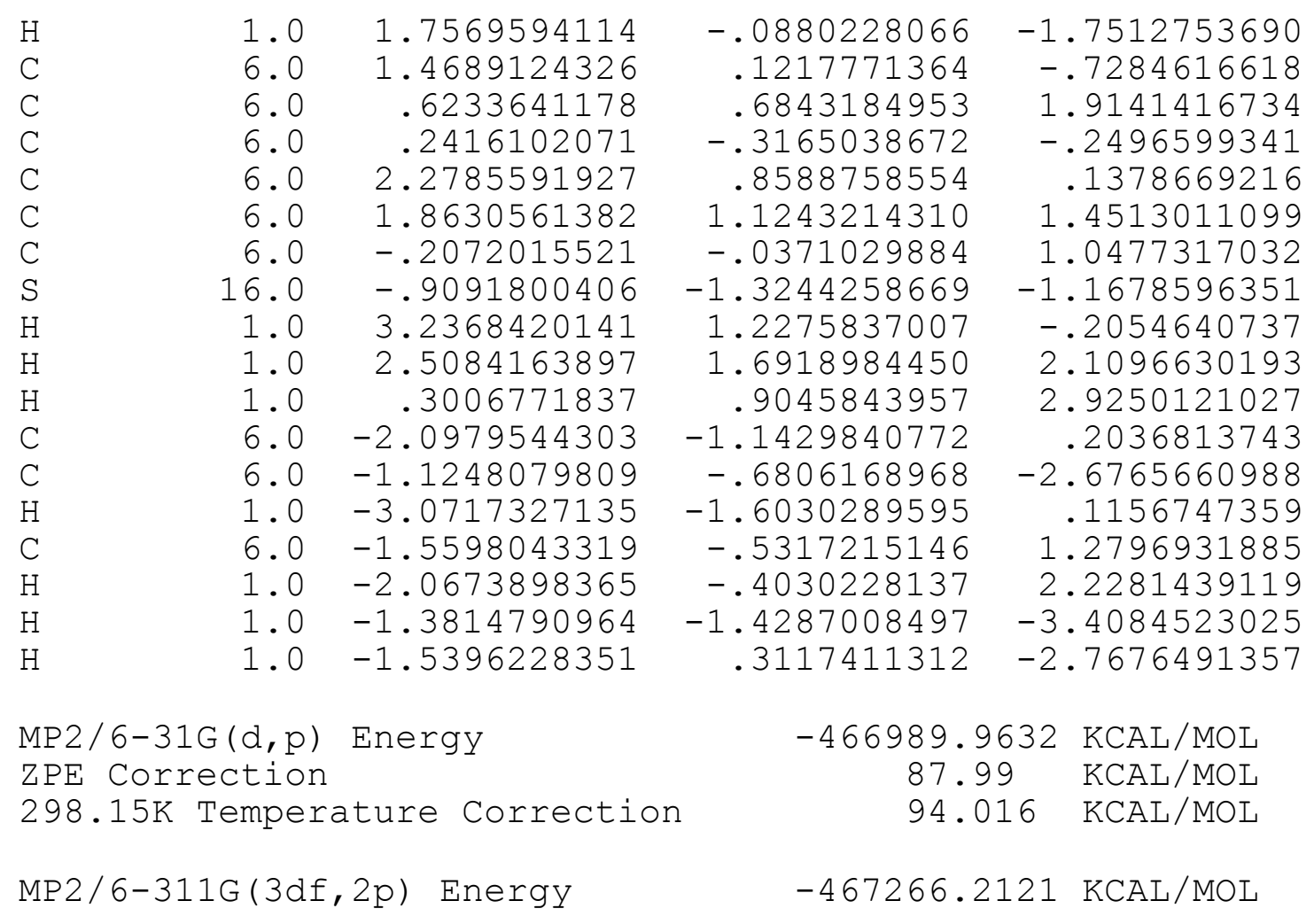




\begin{tabular}{|c|c|c|c|c|}
\hline ATOM & MULL. POP . & CHARGE & LOW. POP. & CHARGE \\
\hline $1 \mathrm{H}$ & .848747 & .151253 & .872670 & .127330 \\
\hline $2 \mathrm{C}$ & 6.103948 & -.103948 & 6.094875 & -.094875 \\
\hline 3 & 6.114958 & -.114958 & 6.106817 & -.106817 \\
\hline 4 & 6.227225 & -.227225 & 6.159744 & -.159744 \\
\hline 5 & 6.113999 & -.113999 & 6.103329 & -.103329 \\
\hline 6 & 6.127378 & -.127378 & 6.110338 & -.110338 \\
\hline 7 & 5.956037 & .043963 & 6.020122 & -.020122 \\
\hline 8 & 15.472159 & 527841 & 15.328978 & .671022 \\
\hline 9 & .870651 & .129349 & .883077 & .116923 \\
\hline 10 & .872849 & .127151 & .882083 & .117917 \\
\hline 11 & .872253 & .127747 & .885224 & .114776 \\
\hline 12 & 6.348089 & -.348089 & 6.284370 & -.284370 \\
\hline 13 & 6.535781 & -.535781 & 6.648509 & -.648509 \\
\hline 14 & .852070 & .147930 & .868845 & .131155 \\
\hline 15 & 6.056973 & -.056973 & 6.106447 & -.106447 \\
\hline 16 & .860489 & .139511 & .878006 & .121994 \\
\hline 17 & $.8793^{\circ}$ & .120627 & .876526 & .123474 \\
\hline 18 & .887021 & .112979 & .890041 & .10995 \\
\hline
\end{tabular}

\section{dibenzothiophene $S, C$-methylide (26)}

MP2 / 6-31G (d, p)

$\begin{array}{rrrrr}\mathrm{H} & 1.0 & -2.4446278123 & -1.8562766526 & -1.8378993925 \\ \mathrm{C} & 6.0 & -2.1717556951 & -1.5511436585 & -.8337683207 \\ \mathrm{C} & 6.0 & -1.4330426480 & -.7449121422 & 1.7713881305 \\ \mathrm{C} & 6.0 & -.9670219665 & -.9012345336 & -.5947924753 \\ \mathrm{C} & 6.0 & -3.0212374450 & -1.7873873060 & .2497292105 \\ \mathrm{C} & 6.0 & -2.6478658534 & -1.3914480627 & 1.5417410644 \\ \mathrm{C} & 6.0 & -.5873399128 & -.4816758047 & .6869315233 \\ \mathrm{~S} & 16.0 & .3219490305 & -.5609018491 & -1.8682822613 \\ \mathrm{H} & 1.0 & -3.9734223942 & -2.2788853142 & .0934102734 \\ \mathrm{H} & 1.0 & -3.3129498850 & -1.5885974751 & 2.3732247088 \\ \mathrm{H} & 1.0 & -1.1573001337 & -.4429038905 & 2.7751043271 \\ \mathrm{C} & 6.0 & 1.2438754222 & . .3232726024 & -.6068921049 \\ \mathrm{C} & 6.0 & .0622565085 & .4547008238 & -3.1438101772 \\ \mathrm{C} & 6.0 & 2.5567031558 & 1.5169524804 & 1.5043999676 \\ \mathrm{C} & 6.0 & 2.4176211525 & 1.0186410931 & -.8679786981 \\ \mathrm{C} & 6.0 & .6981590787 & .2215414560 & .6795162284 \\ \mathrm{C} & 6.0 & 1.3709336289 & .8244465184 & 1.7491151061 \\ \mathrm{C} & 6.0 & 3.0709242390 & 1.6275863686 & . .2049560089 \\ \mathrm{H} & 1.0 & 2.7815248140 & 1.0984947263 & -1.8850763377 \\ \mathrm{H} & 1.0 & .9739452990 & .7609407683 & 2.7552948187 \\ \mathrm{H} & 1.0 & 3.9833653469 & 2.1847370211 & .0343140838 \\ \mathrm{H} & 1.0 & 3.0781858718 & 1.9899590141 & 2.3269555808 \\ \mathrm{H} & 1.0 & -.0411161136 & -.0710806929 & -4.0787089721 \\ \mathrm{H} & 1.0 & -.4717976985 & 1.3800501807 & -2.9893083205\end{array}$


$\operatorname{MP} 2 / 6-31 G(d, p)$ Energy

298.15K Temperature Correction

MP2/6-311G (3df,2p) Energy
ZPE Correction

$\begin{array}{cc}-563119.5975 & \mathrm{KCAL} / \mathrm{MOL} \\ 117.32 & \mathrm{KCAL} / \mathrm{MOL} \\ 125.239 & \mathrm{KCAL} / \mathrm{MOL} \\ -563489.0401 & \mathrm{KCAL} / \mathrm{MOL}\end{array}$

TOTAL MULLIKEN AND LOWDIN ATOMIC POPULATIONS

\begin{tabular}{|c|c|c|c|c|}
\hline ATOM & MULL. POP & CHARGE & LOW. POP. & CHARGE \\
\hline $1 \mathrm{H}$ & .905468 & .094532 & .934994 & .065006 \\
\hline $2 \mathrm{C}$ & 6.065510 & -.065510 & 6.056461 & -.056461 \\
\hline $\mathrm{C}$ & 6.031825 & -.031825 & 6.052638 & -.052638 \\
\hline $\mathrm{C}$ & 6.234702 & -.234702 & 6.203005 & -.203005 \\
\hline $\mathrm{C}$ & 6.070909 & -.070909 & 6.051876 & -.051876 \\
\hline C & 6.095908 & -.095908 & 6.058732 & -.058732 \\
\hline $\mathrm{C}$ & 5.971702 & .028298 & 6.023972 & -.023972 \\
\hline S & 15.522559 & .477441 & 15.195129 & .804871 \\
\hline $\mathrm{H}$ & .901351 & .098649 & .934525 & .065475 \\
\hline 10 & .902390 & .097610 & .932731 & .067269 \\
\hline 11 & .911140 & .088860 & .937324 & .062676 \\
\hline 12 & 6.226751 & -.226751 & 6.184757 & -.184757 \\
\hline 13 & 6.591037 & -.591037 & 6.580098 & -.580098 \\
\hline 14 & 6.094762 & -.094762 & 6.059275 & -.059275 \\
\hline 15 & 6.036314 & -.036314 & 6.049370 & -.049370 \\
\hline 16 & 5.992240 & .007760 & 6.024301 & -.024301 \\
\hline 17 & 6.033714 & -.033714 & 6.051622 & -.051622 \\
\hline 18 & 6.070838 & -.070838 & 6.050522 & -.050522 \\
\hline 19 & .894389 & .105611 & .930812 & .069188 \\
\hline 20 & .910476 & .089524 & .936918 & .063082 \\
\hline 21 & .899289 & .100711 & .933104 & .066896 \\
\hline 22 & .902356 & .097644 & .932109 & .067891 \\
\hline 23 & .859080 & .140920 & .937195 & .062805 \\
\hline $24 \mathrm{H}$ & .875289 & .124711 & .948530 & .051470 \\
\hline
\end{tabular}

dimethyl selenoxide (27)

$\operatorname{MP} 2 / 6-31 \mathrm{~g}(\mathrm{~d}, \mathrm{p})$

$\begin{array}{lrrrr}\mathrm{O} & 8.0 & .8208529984 & -.9001033183 & 1.8200983778 \\ \mathrm{SE} & 34.0 & .9635044466 & .2122385688 & .5999354568 \\ \mathrm{C} & 6.0 & .6144882918 & -.8458651523 & -.9968954576 \\ \mathrm{C} & 6.0 & -.7874339346 & 1.0633275595 & .5786769120 \\ \mathrm{H} & 1.0 & -.8517280888 & 1.6810318258 & 1.4703345958 \\ \mathrm{H} & 1.0 & -1.5207509857 & .2621709362 & .6308749700 \\ \mathrm{H} & 1.0 & -.9141853857 & 1.6725012016 & -.3136604272 \\ \mathrm{H} & 1.0 & -.2395055334 & -1.4764012848 & -.7609458405 \\ \mathrm{H} & 1.0 & 1.4915625420 & -1.4627947097 & -1.1727418037 \\ \mathrm{H} & 1.0 & .4231958095 & -.2061058388 & -1.8556769424\end{array}$

MP2 / 6-31G (d,p) Energy

ZPE Correction

298.15K Temperature Correction $\begin{array}{cc}-1554502.477643 & \mathrm{KCAL} / \mathrm{MOL} \\ 48.4 & \mathrm{KCAL} / \mathrm{MOL} \\ 52.173 & \mathrm{KCAL} / \mathrm{MOL}\end{array}$ 


\begin{tabular}{lcc}
\hline Basis Set & MP2 $(\mathrm{kcal} / \mathrm{mol})$ & B3LYP $(\mathrm{kcal} / \mathrm{mol})$ \\
\hline $6-31 \mathrm{G}(\mathrm{d})$ & -1554471.647425 & -1555616.774921 \\
$6-31 \mathrm{G}(2 \mathrm{~d})$ & -1554571.415981 & -1555693.317194 \\
$6-31 \mathrm{G}(\mathrm{d}, \mathrm{p})$ & -1554502.477643 & -1555622.798761 \\
$6-31+\mathrm{G}(\mathrm{d})$ & -1554485.229319 & -1555629.816120 \\
$6-31+\mathrm{G}(2 \mathrm{~d}, \mathrm{p})$ & -1554610.962258 & -1555708.541470 \\
$6-311 \mathrm{G}(\mathrm{d})$ & -1555874.429914 & -1556973.245152 \\
$6-311 \mathrm{G}(3 \mathrm{df}, 2 \mathrm{p})$ & -1555959.852014 & -1556985.741316 \\
$6-311++\mathrm{G}(\mathrm{d})$ & -1555875.525965 & -1556973.705192 \\
$6-311++\mathrm{G}(3 \mathrm{df}, 2 \mathrm{p})$ & -1555960.544073 & -1556986.234998 \\
\hline G3 (298.15K) & \multicolumn{2}{c}{-1603812.0811} \\
G3 (0K) & -1603815.3824 \\
\hline
\end{tabular}

TOTAL MULLIKEN AND LOWDIN ATOMIC POPULATIONS

\begin{tabular}{rrrrrr} 
ATOM & MULL.POP. & CHARGE & LOW.POP. & \multicolumn{1}{c}{ CHARGE } \\
1 & $\mathrm{O}$ & 8.691582 & -.691582 & 8.658991 & -.658991 \\
2 & $\mathrm{SE}$ & 33.225381 & .774619 & 33.229096 & .770904 \\
$3 \mathrm{C}$ & 6.485990 & -.485990 & 6.430092 & -.430092 \\
4 & $\mathrm{C}$ & 6.485821 & -.485821 & 6.429217 & -.429217 \\
5 & $\mathrm{H}$ & .843368 & .156632 & .871096 & .128904 \\
6 & $\mathrm{H}$ & .843092 & .156908 & .873442 & .126558 \\
7 & $\mathrm{H}$ & .869330 & .130670 & .882091 & .117909 \\
$8 \mathrm{H}$ & .842187 & .157813 & .872741 & .127259 \\
9 & $\mathrm{H}$ & .844044 & .155956 & .871464 & .128536 \\
$10 \mathrm{H}$ & .869206 & .130794 & .881771 & .118229
\end{tabular}

selenirane oxide (28)

$\operatorname{MP} 2 / 6-31 g(d, p)$

$\begin{array}{lrrrr}\mathrm{C} & 6.0 & -.5722063260 & .0364229451 & -1.2608373103 \\ \mathrm{C} & 6.0 & .8950487900 & .0363569995 & -1.0564500619 \\ \mathrm{H} & 1.0 & -1.0909645650 & .9857808610 & -1.2285650228 \\ \mathrm{H} & 1.0 & -1.0191014569 & -.7338039491 & -1.8754086608 \\ \mathrm{H} & 1.0 & 1.3852370708 & .9857175566 & -.8836108083 \\ \mathrm{H} & 1.0 & 1.4928243227 & -.7338385138 & -1.5254685201 \\ \mathrm{SE} & 34.0 & -.0824531030 & -.5073128356 & .5918096339 \\ \mathrm{O} & 8.0 & -.2161467325 & .8291329363 & 1.5517107502\end{array}$

MP2 / 6-31G ( d,p) Energy

ZPE Correction

$-1553743.742 \mathrm{KCAL} / \mathrm{MOL}$

$35.04 \mathrm{KCAL} / \mathrm{MOL}$

298.15K Temperature Correction

$37.809 \mathrm{KCAL} / \mathrm{MOL}$ 


\begin{tabular}{lcc}
\hline Basis Set & MP2 (kcal/mol) & B3LYP (kcal/mol) \\
\hline $6-31 G(d)$ & -1553723.394 & -1554848.268 \\
$6-31 G(2 d)$ & -1553822.846 & -1554925.987 \\
$6-31 G(d, p)$ & -1553743.742 & -1554852.453 \\
$6-31+G(d)$ & -1553738.427 & -1554862.515 \\
$6-31+G(2 d, p)$ & -1553853.067 & -1554940.373 \\
$6-311 G(d)$ & -1555126.673 & -1556206.323 \\
$6-311 G(3 d f, 2 p)$ & -1555200.819 & -1556217.718 \\
$6-311++G(d)$ & -1555127.672 & -1556206.769 \\
$6-311++G(3 d f, 2 p)$ & -1555201.548 & -1556218.267 \\
\hline$G 3(298.15 K)$ & -1603052.7149 \\
$G 3(0 K)$ & -1603055.1942 \\
\hline
\end{tabular}

TOTAL MULLIKEN AND LOWDIN ATOMIC POPULATIONS

$\begin{array}{llrrr}\text { ATOM } & \text { MULL.POP } & \text { CHARGE } & \text { LOW.POP. } & \text { CHARGE } \\ 1 \mathrm{C} & 6.341793 & -.341793 & 6.293027 & -.293027 \\ 2 \mathrm{C} & 6.341795 & -.341795 & 6.291138 & -.291138 \\ 3 \mathrm{H} & .818943 & .181057 & .862484 & .137516 \\ 4 \mathrm{H} & .843319 & .156681 & .872060 & .127940 \\ 5 \mathrm{H} & .818943 & .181057 & .862514 & .137486 \\ 6 \mathrm{H} & .843310 & .156690 & .871994 & .128006 \\ 7 \mathrm{SE} & 33.340530 & .659470 & 33.317216 & .682784 \\ 8 \mathrm{O} & 8.651369 & -.651369 & 8.629568 & -.629568\end{array}$

\section{methyl vinyl selenoxide (29)}

\begin{tabular}{|c|c|c|c|c|}
\hline 0 & 8.0 & -.7959386755 & 1.8732242106 & -1.4601676824 \\
\hline SE & 34.0 & -.6524741597 & .2702255083 & -1.0675310352 \\
\hline $\mathrm{C}$ & 6.0 & -1.6142038901 & .1556069020 & .6224744558 \\
\hline C & 6.0 & 1.0722514415 & .1371171570 & -.2188006147 \\
\hline $\mathrm{H}$ & 1.0 & -1.3939803687 & -.7879915956 & 1.1155043644 \\
\hline $\mathrm{H}$ & 1.0 & -2.6748392218 & .2391058649 & .3995504062 \\
\hline $\mathrm{H}$ & 1.0 & -1.2913968836 & 1.0085544884 & 1.2141288317 \\
\hline $\mathrm{C}$ & 6.0 & 1.7798576093 & -.9946527271 & -.2708557329 \\
\hline $\mathrm{H}$ & 1.0 & 1.4134804744 & 1.0507432235 & .2554242417 \\
\hline $\mathrm{H}$ & 1.0 & 1.4049959700 & -1.8708735585 & -.7846144052 \\
\hline $\mathrm{H}$ & 1.0 & 2.7522477051 & -1.0810592584 & .1948865325 \\
\hline & $\begin{array}{l}(\mathrm{d}, \mathrm{p}) \\
\text { ction } \\
\text { emper }\end{array}$ & ture Correcti & \multicolumn{2}{|c|}{$\begin{array}{r}-1578323.936 \mathrm{KCAL} / \mathrm{MOL} \\
51.54 \mathrm{KCAL} / \mathrm{MOL} \\
55.687 \mathrm{KCAL} / \mathrm{MOL}\end{array}$} \\
\hline
\end{tabular}




\begin{tabular}{lcc}
\hline Basis Set & MP2 $(\mathrm{kcal} / \mathrm{mol})$ & B3LYP $(\mathrm{kcal} / \mathrm{mol})$ \\
\hline $6-31 \mathrm{G}(\mathrm{d})$ & -1578293.073 & -1579496.991 \\
$6-31 \mathrm{G}(2 \mathrm{~d})$ & -1578399.927 & -1579574.889 \\
$6-31 \mathrm{G}(\mathrm{d}, \mathrm{p})$ & -1578323.936 & -1579503.306 \\
$6-31+\mathrm{G}(\mathrm{d})$ & -1578312.804 & -1579515.26 \\
$6-31+\mathrm{G}(2 \mathrm{~d}, \mathrm{p})$ & -1578443.142 & -1579594.083 \\
$6-311 \mathrm{G}(\mathrm{d})$ & -1579704.734 & -1580859.329 \\
$6-311 \mathrm{G}(3 \mathrm{df}, 2 \mathrm{p})$ & -1579804.663 & -1580874.795 \\
$6-311++\mathrm{G}(\mathrm{d})$ & -1579706.791 & -1580860.414 \\
$6-311++\mathrm{G}(3 \mathrm{df}, 2 \mathrm{p})$ & -1579806.047 & -1580875.823 \\
\hline G3 (298.15K) & -1627690.7725 \\
G3 (0K) & -1627694.4666 \\
\hline
\end{tabular}

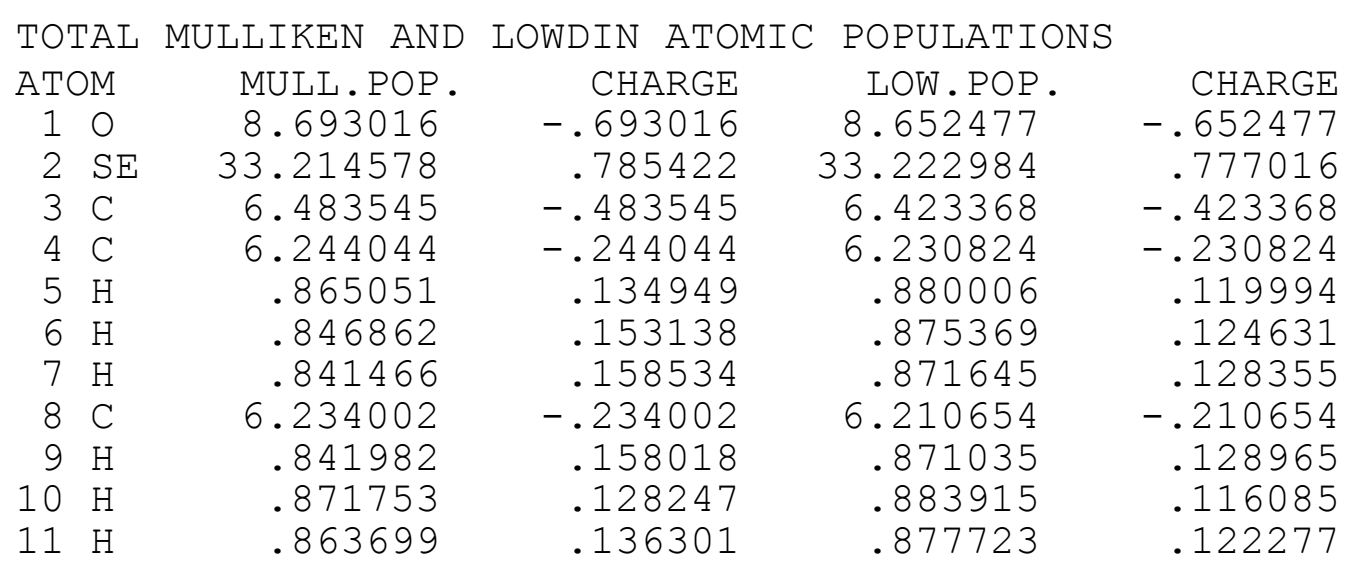

selenophene-Se-oxide (30)

\begin{tabular}{|c|c|c|c|c|c|}
\hline C & 6.0 & 1.2816361341 & -.3548905868 & & 1317331837 \\
\hline C & 6.0 & .7019857259 & .1219183535 & & 9905357023 \\
\hline C & 6.0 & -.7432991567 & .3351393742 & & 9074391717 \\
\hline $\mathrm{C}$ & 6.0 & -1.3064707862 & .0266361110 &.- & 2802020659 \\
\hline & 1.0 & 2.3245242242 & -.5898350287 &.- & 2784649430 \\
\hline $\mathrm{H}$ & 1.0 & 1.2555634058 & .3494343250 & 1. & 8928589365 \\
\hline $\mathrm{H}$ & 1.0 & -1.3052257248 & .7283700519 & 1 . & 7451055142 \\
\hline $\mathrm{H}$ & 1.0 & -2.3495913162 & .0999037527 & - & 5465290752 \\
\hline SE & 34.0 & -.0358897283 & -.8125356673 & -1 & 4643953442 \\
\hline & 8.0 & .1767442031 & .0958442355 & -2 & 8345410517 \\
\hline \multirow{3}{*}{\multicolumn{2}{|c|}{$\begin{array}{l}\mathrm{MP} 2 / 6-31 \mathrm{G}(\mathrm{d}, \mathrm{p}) \\
\mathrm{ZPE} \text { Correction } \\
298.15 \mathrm{~K} \text { Tempera }\end{array}$}} & Energy & \multicolumn{2}{|c|}{-1648457.0292} & $\mathrm{KCAL} / \mathrm{MOL}$ \\
\hline & & & \multicolumn{2}{|c|}{43.22} & KCAL/MOL \\
\hline & & ature Correction & \multicolumn{2}{|c|}{47.427} & KCAL/MOL \\
\hline \multicolumn{3}{|c|}{ MP2/6-311G(3df,2p) Energy } & \multicolumn{2}{|c|}{-1650023.469} & $\mathrm{KCAL} / \mathrm{MOI}$ \\
\hline
\end{tabular}




$\begin{array}{rrrrrr}\text { TOTAL } & \text { MULLIKEN AND } & \text { LOWDIN ATOMIC } & \text { POPULATIONS } & \\ \text { ATOM } & \text { MULL.POP. } & \text { CHARGE } & \text { LOW.POP. } & \text { CHARGE } \\ 1 \text { C } & 6.293989 & -.293989 & 6.236481 & -.236481 \\ 2 \mathrm{C} & 6.080826 & -.080826 & 6.101541 & -.101541 \\ 3 \mathrm{C} & 6.080882 & -.080882 & 6.101951 & -.101951 \\ 4 & \mathrm{C} & 6.293994 & -.293994 & 6.235965 & -.235965 \\ 5 \mathrm{H} & .840959 & .159041 & .866348 & .133652 \\ 6 \mathrm{H} & .855242 & .144758 & .875217 & .124783 \\ 7 \mathrm{H} & .855277 & .144723 & .874565 & .125435 \\ 8 \mathrm{H} & .840924 & .159076 & .866364 & .133636 \\ 9 & \mathrm{SE} & 33.214375 & .785625 & 33.228131 & .771869 \\ 10 & \mathrm{O} & 8.643532 & -.643532 & 8.613437 & -.613437\end{array}$

\section{benzoselenophene-Se-oxide (31)}

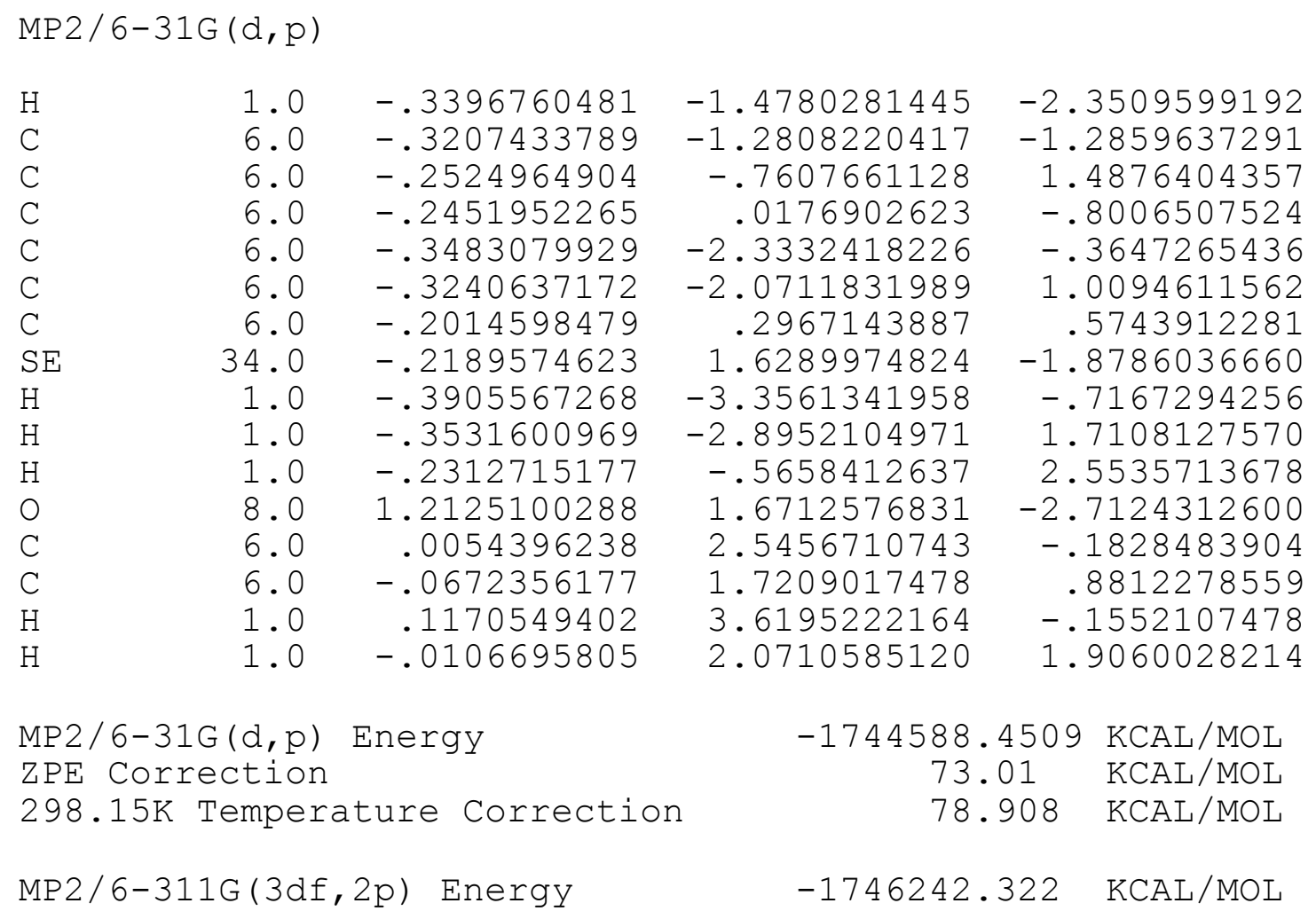




\begin{tabular}{|c|c|c|c|c|}
\hline ATOM & MULL . POP . & CHARGE & LOW. POP. & CHARGE \\
\hline $1 \mathrm{H}$ & .852959 & .147041 & .878240 & .121760 \\
\hline $\mathrm{C}$ & 6.118780 & -.118780 & 6.095854 & -.095854 \\
\hline $\mathrm{C}$ & 6.115113 & -.115113 & 6.101791 & -.101791 \\
\hline $\mathrm{C}$ & 6.194052 & -.194052 & 6.133743 & -.133743 \\
\hline $\mathrm{C}$ & 6.112724 & -.112724 & 6.098747 & -.098747 \\
\hline $\mathrm{C}$ & 6.123479 & -.123479 & 6.103045 & -.103045 \\
\hline $\mathrm{C}$ & 5.967497 & .032503 & 6.017910 & -.017910 \\
\hline $\mathrm{SE}$ & 33.190254 & .809746 & 33.230071 & .769929 \\
\hline $\mathrm{H}$ & .865788 & .134212 & .881853 & .118147 \\
\hline 10 & .867509 & .132491 & .880508 & .119492 \\
\hline 11 & .867437 & .132563 & .884415 & .115585 \\
\hline 12 & 8.649557 & -.649557 & 8.617166 & -.617166 \\
\hline 13 & 6.322363 & -.322363 & 6.239106 & -.239106 \\
\hline 14 & 6.050411 & -.050411 & 6.089845 & -.089845 \\
\hline $\mathrm{H}$ & .844720 & .155280 & .869740 & .130260 \\
\hline $\mathrm{H}$ & 57359 & .142641 & 877966 & .122034 \\
\hline
\end{tabular}

dibenzoselenophene-Se-oxide (32)

MP2 / 6-31G (d,p)

\begin{tabular}{|c|c|c|c|c|c|}
\hline 0 & 8.0 & -1.8775564466 & .0005392725 & \multicolumn{2}{|c|}{-2.4117512446} \\
\hline SE & 34.0 & -.3297641113 & -.0000284436 & \multicolumn{2}{|c|}{-1.8218138627} \\
\hline C & 6.0 & -.2376360463 & -1.3049193542 & \multicolumn{2}{|c|}{-.3853360889} \\
\hline C & 6.0 & -.2373929240 & 1.3048029007 & \multicolumn{2}{|c|}{-.3853564212} \\
\hline $\mathrm{C}$ & 6.0 & -.0672601548 & -2.9627735106 & \multicolumn{2}{|c|}{1.8204094796} \\
\hline C & 6.0 & -.3088152624 & -2.6804844360 & \multicolumn{2}{|c|}{-.5742265582} \\
\hline $\mathrm{C}$ & 6.0 & -.0952376515 & -.7346207063 & \multicolumn{2}{|c|}{.8901069486} \\
\hline C & 6.0 & .0019513778 & -1.5802962308 & \multicolumn{2}{|c|}{2.0006359495} \\
\hline C & 6.0 & -.2283008434 & -3.5138331743 & \multicolumn{2}{|c|}{.5442903673} \\
\hline $\mathrm{H}$ & 1.0 & -.4348 & -3.0982 & \multicolumn{2}{|c|}{-1.5658978583} \\
\hline $\mathrm{H}$ & 1.0 & & -1.1706 & \multicolumn{2}{|c|}{2.9968118905} \\
\hline $\mathrm{H}$ & 1.0 & -.290 & -4.5878 & \multicolumn{2}{|c|}{.4237209654} \\
\hline $\mathrm{H}$ & 1.0 & 45299 & -3.6166 & \multicolumn{2}{|c|}{2.6806602412} \\
\hline $\mathrm{C}$ & 6.0 & -.0672 & 51727 & \multicolumn{2}{|c|}{1.8204146874} \\
\hline $\mathrm{C}$ & 6.0 & 00624 & 6517 & \multicolumn{2}{|c|}{.8900777776} \\
\hline $\mathrm{C}$ & 6.0 & -.3087 & 3719 & \multicolumn{2}{|c|}{-.5742445654} \\
\hline $\mathrm{C}$ & 6.0 & -.2283 & 1833 & \multicolumn{2}{|c|}{.5442289238} \\
\hline $\mathrm{C}$ & 6.0 & & 1.580 & \multicolumn{2}{|c|}{2.0006601487} \\
\hline $\mathrm{H}$ & 1.0 & -.43 & 3644 & \multicolumn{2}{|c|}{-1.5659755792} \\
\hline $\mathrm{H}$ & 1.0 & -.29 & 57162 & \multicolumn{2}{|c|}{.4237030788} \\
\hline $\mathrm{H}$ & 1.0 & & 8745 & \multicolumn{2}{|c|}{2.9967863560} \\
\hline $\mathrm{H}$ & 1.0 & & 3.616 & \multicolumn{2}{|c|}{2.6806517290} \\
\hline \\
\hline \multicolumn{3}{|c|}{$\begin{array}{l}\mathrm{MP} 2 / 6-31 \mathrm{G}(\mathrm{d}, \mathrm{p}) \quad \text { Energy } \\
\mathrm{ZPF} \text { Correction }\end{array}$} & \multicolumn{2}{|c|}{$\begin{array}{c}-1840719.9327 \\
102.45\end{array}$} & \\
\hline \multicolumn{3}{|c|}{$\begin{array}{l}\text { ZPE Correction } \\
298.15 \mathrm{~K} \text { Temperature Correction }\end{array}$} & \multicolumn{2}{|c|}{$\begin{array}{l}102.45 \\
110.225\end{array}$} & $\mathrm{KCAL} / \mathrm{MOI}$ \\
\hline \multicolumn{3}{|c|}{ MP2/6-311G (3df,2p) Energy } & \multicolumn{2}{|c|}{-1842468.382} & KCAI \\
\hline
\end{tabular}




$\begin{array}{rlrrrr}\text { TOTAL } & \text { MULLIKEN AND } & \text { LOWDIN ATOMIC } & \text { POPULATIONS } & \\ \text { ATOM } & \text { MULL.POP. } & \text { CHARGE } & \text { LOW.POP. } & \text { CHARGE } \\ 1 & \mathrm{O} & 8.651418 & -.651418 & 8.615908 & -.615908 \\ 2 & \mathrm{SE} & 33.168517 & .831483 & 33.236655 & .763345 \\ 3 \mathrm{C} & 6.206091 & -.206091 & 6.133786 & -.133786 \\ 4 & \mathrm{C} & 6.206076 & -.206076 & 6.133776 & -.133776 \\ 5 & \mathrm{C} & 6.131054 & -.131054 & 6.102445 & -.102445 \\ 6 & \mathrm{C} & 6.126939 & -.126939 & 6.100012 & -.100012 \\ 7 & \mathrm{C} & 5.958020 & .041980 & 6.010975 & -.010975 \\ 8 & \mathrm{C} & 6.100881 & -.100881 & 6.102766 & -.102766 \\ 9 & \mathrm{C} & 6.107714 & -.107714 & 6.097265 & -.097265 \\ 10 & \mathrm{H} & .854837 & .145163 & .877557 & .122443 \\ 11 & \mathrm{H} & .869538 & .130462 & .884371 & .115629 \\ 12 & \mathrm{H} & .866630 & .133370 & .883378 & .116622 \\ 13 & \mathrm{H} & .868370 & .131630 & .881173 & .118827 \\ 14 & \mathrm{C} & 6.131057 & -.131057 & 6.102447 & -.102447 \\ 15 & \mathrm{C} & 5.957986 & .042014 & 6.010976 & -.010976 \\ 16 & \mathrm{C} & 6.126925 & -.126925 & 6.099997 & -.099997 \\ 17 & \mathrm{C} & 6.107714 & -.107714 & 6.097266 & -.097266 \\ 18 & \mathrm{C} & 6.100859 & -.100859 & 6.102772 & -.102772 \\ 19 & \mathrm{H} & .854832 & .145168 & .877554 & .122446 \\ 20 & \mathrm{H} & .866631 & .133369 & .883378 & .116622 \\ 21 & \mathrm{H} & .869543 & .130457 & .884372 & .115628 \\ 22 & \mathrm{H} & .868367 & .131633 & .881172 & .118828\end{array}$

\section{$N$-formyl-S,S-dimethylsulfilimine (33)}

$\operatorname{MP} 2 / 6-31 G(d, p)$

$\begin{array}{lrr}\mathrm{N} & 7.0 & .1359908717 \\ \mathrm{~S} & 16.0 & .0879343664 \\ \mathrm{C} & 6.0 & 1.1124330185 \\ \mathrm{C} & 6.0 & -1.5187306304 \\ \mathrm{H} & 1.0 & -1.5730397327 \\ \mathrm{H} & 1.0 & -2.2650682487 \\ \mathrm{H} & 1.0 & -1.6610561413 \\ \mathrm{H} & 1.0 & 2.1447358810 \\ \mathrm{H} & 1.0 & .9674726470 \\ \mathrm{H} & 1.0 & .8472194743 \\ \mathrm{C} & 6.0 & .4789406869 \\ \mathrm{H} & 1.0 & .6658588326 \\ \mathrm{O} & 8.0 & .5772689749\end{array}$

MP2 / 6-31G (d,p) Energy

ZPE Correction

298.15K Temperature Correction

MP2 / 6-311G (3df,2p) Energy

$\begin{array}{rr}.5476199662 & -1.1985811673 \\ -.5256491625 & .0286680160 \\ .2396978552 & 1.2931183453 \\ -.2288640660 & .7797752409 \\ -.7174708177 & 1.7507189217 \\ -.6448187691 & .1089864813 \\ .8461558236 & .8660520630 \\ .1403147800 & .9699370556 \\ -.2645551371 & 2.2466079441 \\ 1.2929072372 & 1.3551902474 \\ -.0653960435 & -2.3952392135 \\ -1.1620466630 & -2.3517384983 \\ .5421049966 & -3.4534854362 \\ -404954.7689 & \mathrm{KCAL} / \mathrm{MOL} \\ 65.06413 \mathrm{KCAL} / \mathrm{MOL} \\ 70.519 & \mathrm{KCAL} / \mathrm{MOL}\end{array}$




\begin{tabular}{|c|c|c|c|c|}
\hline ATON & MULL. POP. & CHARGE & LOW. POP. & CHARGE \\
\hline 11 & 7.621051 & -.621051 & 7.547406 & -.547406 \\
\hline $2 s$ & 15.318740 & .681260 & 15.234004 & .765996 \\
\hline $3 c$ & 6.493699 & -.493699 & 6.422436 & -.422436 \\
\hline $4 c$ & 6.493659 & -.493659 & 6.424433 & -.424433 \\
\hline $5 \mathrm{H}$ & .853203 & .146797 & .872688 & .127312 \\
\hline $6 \mathrm{H}$ & .824099 & .175901 & .857158 & .142842 \\
\hline $7 \mathrm{H}$ & .821314 & .178686 & .866310 & .133690 \\
\hline 8 & .824109 & .175891 & .859874 & .140126 \\
\hline $9 \mathrm{~F}$ & .853225 & .146775 & .872286 & .127714 \\
\hline $10 \mathrm{H}$ & .821332 & .178668 & .865704 & .134296 \\
\hline 110 & 5.666209 & .333791 & 5.941585 & .058415 \\
\hline 12 & .968621 & .031379 & .957225 & .042775 \\
\hline $13 c$ & 8.440738 & -.440738 & 8.278891 & -.278891 \\
\hline
\end{tabular}

\section{$N$-formyl thiophenesulfilimine (34)}

MP2 / 6-31G (d,p)

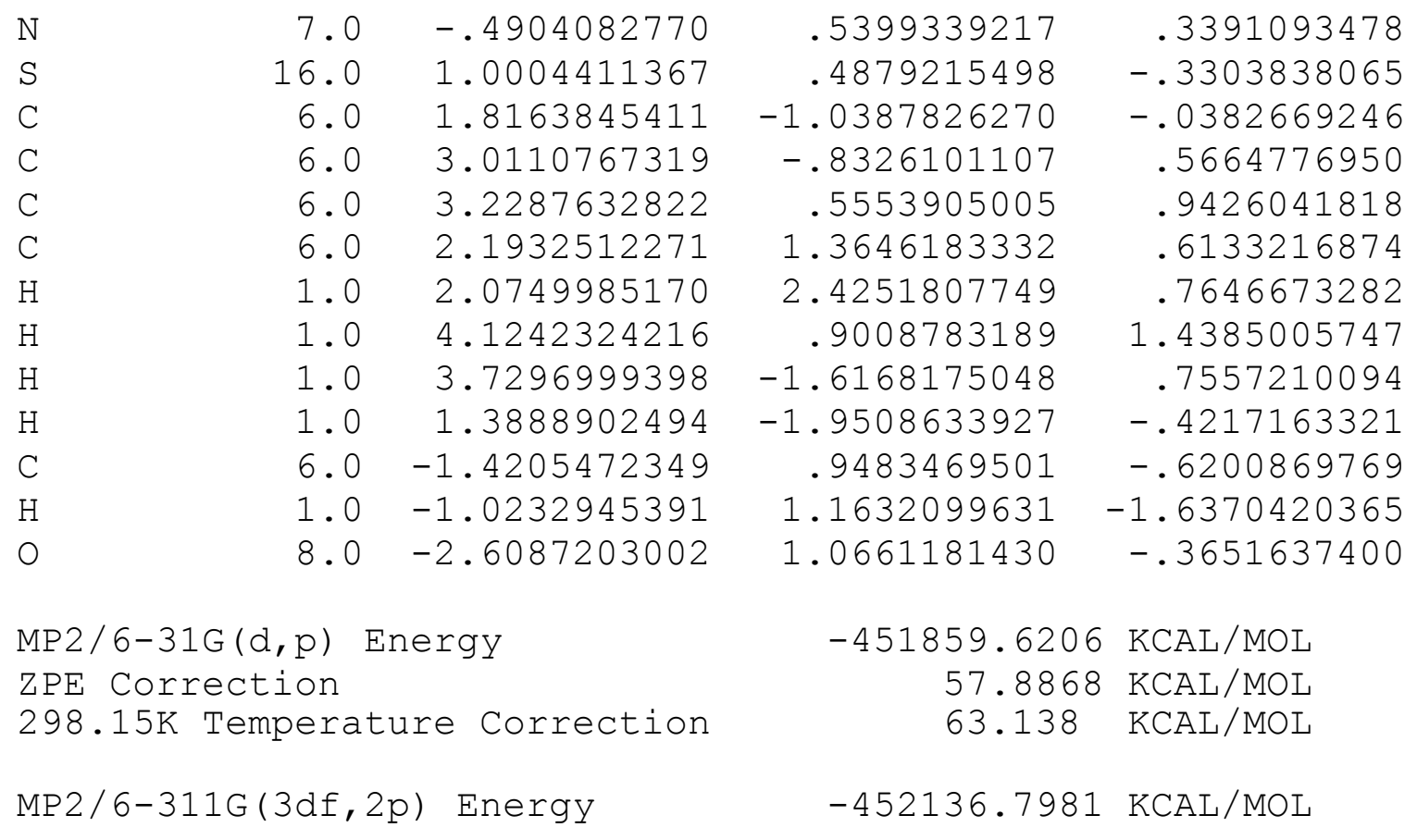




\begin{tabular}{|c|c|c|c|c|}
\hline ATOI & MULL. POP. & CHARGE & LOW . POP. & CHARGE \\
\hline 1 & 7.569965 & -.569965 & 7.508114 & -.508114 \\
\hline 2 & 15.272297 & .727703 & 15.198368 & .801632 \\
\hline 3 & 6.326557 & -.326557 & 6.261934 & -.261934 \\
\hline 4 & 6.078314 & -.078314 & 6.096347 & -.096347 \\
\hline 5 & 6.078440 & -.078440 & 6.095032 & -.095032 \\
\hline 6 & 6.326497 & -.326497 & 6.258011 & -.258011 \\
\hline 7 & .811865 & .188135 & .851782 & .148218 \\
\hline 8 & .838219 & .161781 & .865918 & .134082 \\
\hline 9 & .838195 & .161805 & .865026 & .134974 \\
\hline 10 & .811857 & .188143 & .849073 & .150927 \\
\hline 11 & 5.662368 & .337632 & 5.943749 & .056251 \\
\hline 12 & .959874 & .040126 & .949272 & .050728 \\
\hline 13 & 8.425552 & -.425552 & 8.257374 & -.257374 \\
\hline
\end{tabular}

\section{N-formyl dibenzothiophenesulfilimine (35)}

$\begin{array}{lrrrr}\mathrm{H} & 1.0 & -0.5614045386 & -1.3372427924 & -3.2697609724 \\ \mathrm{C} & 6.0 & -0.3789573238 & -1.6311110177 & -2.2435537460 \\ \mathrm{C} & 6.0 & 0.1204423090 & -2.3584150723 & 0.4437382630 \\ \mathrm{C} & 6.0 & -0.0325840949 & -2.9432369815 & -1.9137546134 \\ \mathrm{C} & 6.0 & -0.4419012821 & -0.7017277752 & -1.2129892499 \\ \mathrm{C} & 6.0 & -0.2030070038 & -1.0350632570 & 0.1287651112 \\ \mathrm{C} & 6.0 & 0.2018163111 & -3.3027081605 & -0.5803055895 \\ \mathrm{H} & 1.0 & 0.0434555385 & -3.6900662106 & -2.6933621837 \\ \mathrm{C} & 6.0 & -0.2964384829 & 0.1248206607 & 1.0128024642 \\ \mathrm{H} & 1.0 & 0.4603308466 & -4.3264708714 & -0.3418711399 \\ \mathrm{H} & 1.0 & 0.3118139595 & -2.6481966409 & 1.4698786919 \\ \mathrm{C} & 6.0 & -0.4788681695 & 2.5993170278 & 2.3111733997 \\ \mathrm{C} & 6.0 & -0.0855356376 & 0.1989726709 & 2.3930388046 \\ \mathrm{C} & 6.0 & -0.6029767616 & 1.3025927153 & 0.3146671152 \\ \mathrm{C} & 6.0 & -0.7146165038 & 2.5400978861 & 0.9359237856 \\ \mathrm{C} & 6.0 & -0.1798027788 & 1.4359243708 & 3.0318158160 \\ \mathrm{H} & 1.0 & 0.1541139782 & -0.6917471401 & 2.9611896494 \\ \mathrm{~S} & 16.0 & -0.9604662494 & 1.0042391497 & -1.4187693133 \\ \mathrm{H} & 1.0 & -0.9455393688 & 3.4306402482 & 0.3646946123 \\ \mathrm{H} & 1.0 & -0.0087452102 & 1.4993232375 & 4.0988062370 \\ \mathrm{H} & 1.0 & -0.5394187942 & 3.5498687161 & 2.8252875994 \\ \mathrm{~N} & 7.0 & -0.0005279553 & 1.8564109139 & -2.4351892533 \\ \mathrm{C} & 6.0 & 1.3403792304 & 1.7309835486 & -2.1299928896 \\ \mathrm{O} & 8.0 & 1.8508880137 & 1.0755432092 & -1.2164741640 \\ \mathrm{H} & 1.0 & 1.9475495783 & 2.3172516646 & -2.8357575044\end{array}$




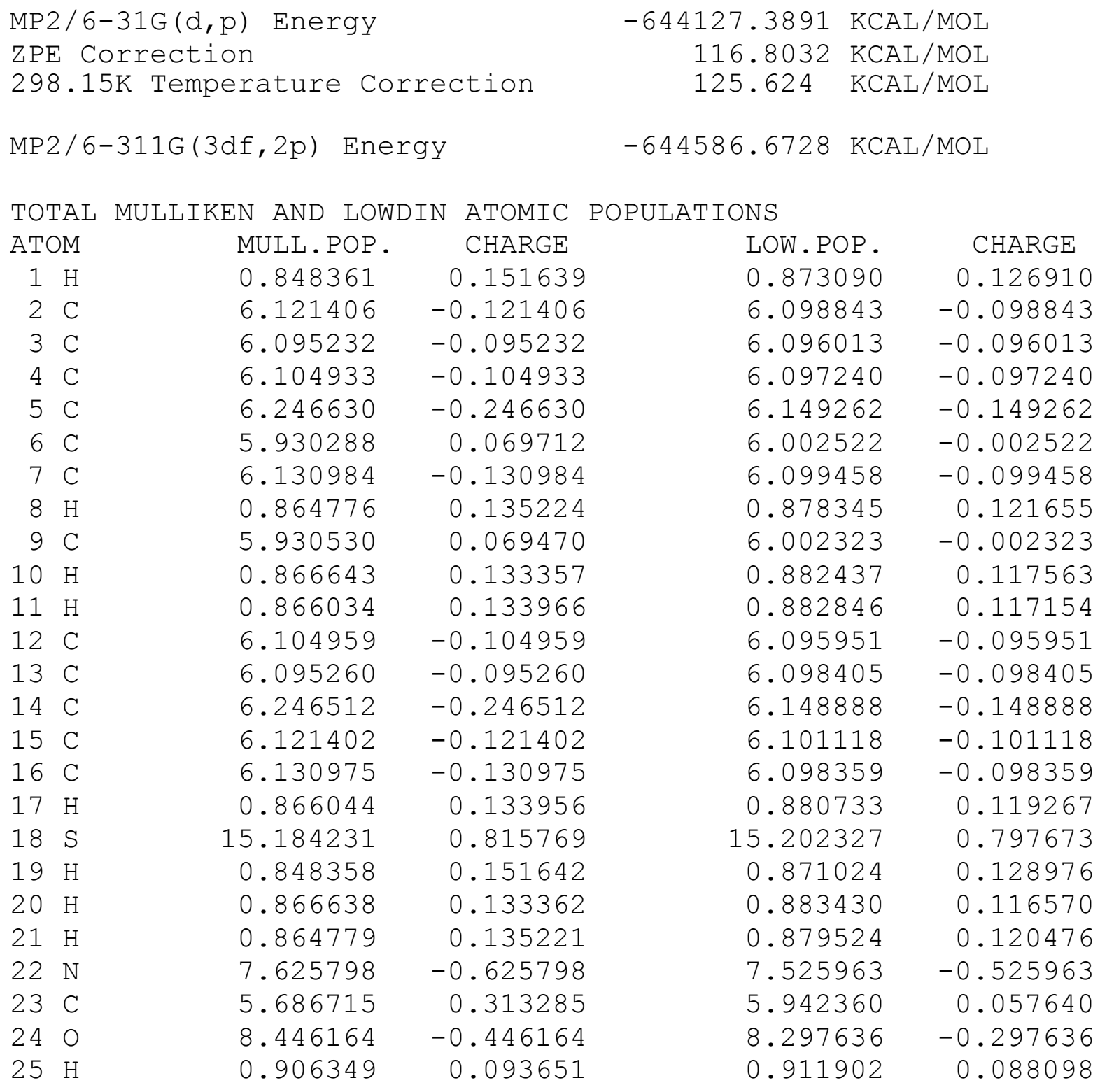




\section{$N$-mesyl-S, S-dimethylsulfilimine (36)}

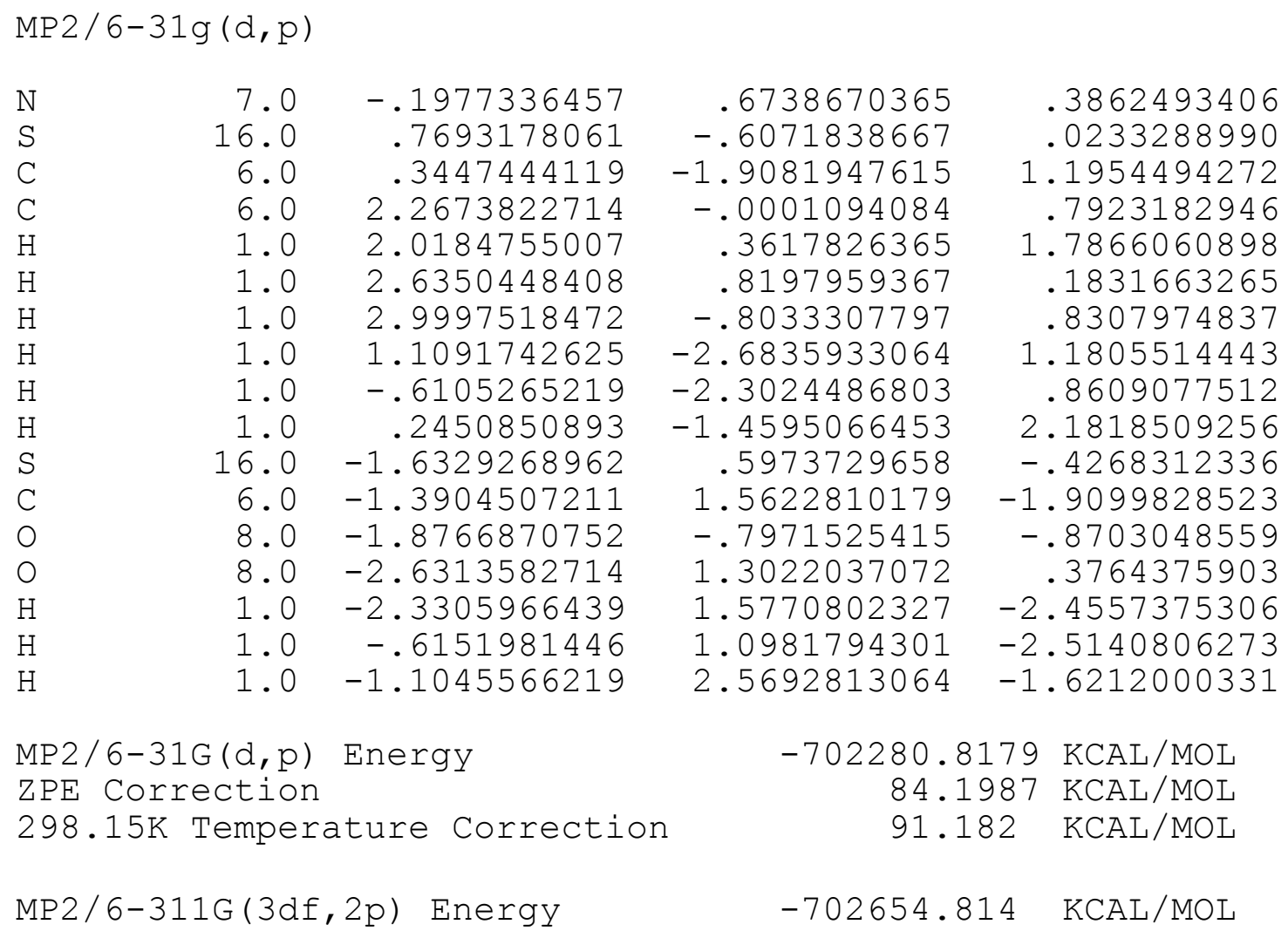

TOTAL MULLIKEN AND LOWDIN ATOMIC POPULATIONS

\begin{tabular}{rrrrrr} 
ATOM & MULL.POP & \multicolumn{1}{c}{ CHARGE } & \multicolumn{1}{c}{ LOW.POP. } & \multicolumn{1}{c}{ CHARGE } \\
1 & $\mathrm{~N}$ & 7.802614 & -0.802614 & 7.795078 & -0.795078 \\
2 & $\mathrm{~S}$ & 15.263627 & 0.736373 & 15.206299 & 0.793701 \\
3 & $\mathrm{C}$ & 6.514292 & -0.514292 & 6.431407 & -0.431407 \\
4 & $\mathrm{C}$ & 6.496793 & -0.496793 & 6.411063 & -0.411063 \\
5 & $\mathrm{H}$ & 0.816007 & 0.183993 & 0.861262 & 0.138738 \\
6 & $\mathrm{H}$ & 0.820657 & 0.179343 & 0.855393 & 0.144607 \\
7 & $\mathrm{H}$ & 0.847155 & 0.152845 & 0.869277 & 0.130723 \\
8 & $\mathrm{H}$ & 0.854925 & 0.145075 & 0.871591 & 0.128409 \\
9 & $\mathrm{H}$ & 0.789871 & 0.210129 & 0.845114 & 0.154886 \\
10 & $\mathrm{H}$ & 0.828099 & 0.171901 & 0.867112 & 0.132888 \\
11 & $\mathrm{~S}$ & 14.762480 & 1.237520 & 14.679469 & 1.320531 \\
12 & $\mathrm{C}$ & 6.524394 & -0.524394 & 6.460316 & -0.460316 \\
13 & $\mathrm{O}$ & 8.615744 & -0.615744 & 8.651433 & -0.651433 \\
14 & $\mathrm{O}$ & 8.535824 & -0.535824 & 8.598175 & -0.598175 \\
15 & $\mathrm{H}$ & 0.832929 & 0.167071 & 0.861151 & 0.138849 \\
16 & $\mathrm{H}$ & 0.856706 & 0.143294 & 0.871606 & 0.128394 \\
17 & $\mathrm{H}$ & 0.837885 & 0.162115 & 0.864253 & 0.135747
\end{tabular}




\section{N-mesyl thiophenesulfilimine (37)}

$\begin{array}{lrr}\mathrm{H} & 1.0 & -1.4794772163 \\ \mathrm{H} & 1.0 & -2.0065402528 \\ \mathrm{C} & 6.0 & -0.8027232900 \\ \mathrm{C} & 6.0 & -1.0352588883 \\ \mathrm{C} & 6.0 & 0.1576269845 \\ \mathrm{H} & 1.0 & 0.1513590339 \\ \mathrm{C} & 6.0 & 1.2739590138 \\ \mathrm{H} & 1.0 & 2.2885524618 \\ \mathrm{~S} & 16.0 & 0.8527232529 \\ \mathrm{~N} & 7.0 & 1.7157232309 \\ \mathrm{~S} & 16.0 & 1.7456482309 \\ \mathrm{C} & 6.0 & 2.7594081841 \\ \mathrm{O} & 8.0 & 0.4071038043 \\ \mathrm{O} & 8.0 & 2.4780915250 \\ \mathrm{H} & 1.0 & 3.7205256066 \\ \mathrm{H} & 1.0 & 2.2541302176 \\ \mathrm{H} & 1.0 & 2.8781952861\end{array}$

MP2/6-31G(d,p) Energy
ZPE Correction
$298.15 \mathrm{~K}$ Temperature Correction

$-749189.1357 \mathrm{KCAL} / \mathrm{MOL}$ $76.7419 \mathrm{KCAL} / \mathrm{MOL}$ $83.667 \mathrm{KCAL} / \mathrm{MOL}$

MP2 / 6-311G (3df,2p) Energy

$-749604.864 \mathrm{KCAL} / \mathrm{MOL}$

\begin{tabular}{|c|c|c|c|c|}
\hline ATOI & MULL. POP. & CHARGE & LOW. POP. & CHARGE \\
\hline & 0.808844 & 0.191156 & 0.846503 & 0.153497 \\
\hline 2 & 0.840553 & 0.159447 & 0.867203 & 0.132797 \\
\hline 3 & 6.315926 & -0.315926 & 6.257001 & -0.257001 \\
\hline 4 & 6.071551 & -0.071551 & 6.094661 & -0.094661 \\
\hline 5 & 6.071746 & -0.071746 & 6.092462 & -0.092462 \\
\hline 6 & 0.840599 & 0.159401 & 0.869440 & 0.130560 \\
\hline 7 & 6.315745 & -0.315745 & 6.254553 & -0.254553 \\
\hline 8 & 0.809080 & 0.190920 & 0.848523 & 0.151477 \\
\hline & 15.258047 & 0.741953 & 15.176330 & 0.823670 \\
\hline 10 & 7.743655 & -0.743655 & 7.750831 & -0.750831 \\
\hline 11 & 14.777256 & 1.222744 & 14.684563 & 1.315437 \\
\hline 12 & 6.535985 & -0.535985 & 6.442270 & -0.442270 \\
\hline 13 & 8.561882 & -0.561882 & 8.619799 & -0.619799 \\
\hline 14 & 8.561740 & -0.561740 & 8.619801 & -0.619801 \\
\hline 15 & 0.828614 & 0.171386 & 0.857999 & 0.142001 \\
\hline & 0.828628 & 0.171372 & 0.857239 & 0.142761 \\
\hline 17 & 0.830152 & 0.169848 & 0.860821 & 0.139179 \\
\hline
\end{tabular}




\section{N-mesyl dibenzothiophenesulfilimine (38)}

\begin{tabular}{|c|c|c|c|c|}
\hline $\mathrm{H}$ & 1.0 & -1.3904225899 & 0.5532307829 & -3.2459595148 \\
\hline C & 6.0 & -1.6780422267 & 0.3931713927 & -2.2140679878 \\
\hline C & 6.0 & -2.3861803629 & -0.0425123898 & 0.4894852303 \\
\hline C & 6.0 & -2.9790029366 & 0.0196496690 & -1.8709148032 \\
\hline C & 6.0 & -0.7473448972 & 0.5024428941 & -1.1891806126 \\
\hline $\mathrm{C}$ & 6.0 & -1.0723624856 & 0.3052169159 & 0.1607698701 \\
\hline $\mathrm{C}$ & 6.0 & -3.3299437265 & -0.1792890679 & -0.5292775602 \\
\hline $\mathrm{H}$ & 1.0 & -3.7257400517 & -0.0997787808 & -2.6452834341 \\
\hline $\mathrm{C}$ & 6.0 & 0.0887736178 & 0.4476108033 & 1.0396258549 \\
\hline $\mathrm{H}$ & 1.0 & -4.3462259357 & -0.4565040866 & -0.2800723491 \\
\hline $\mathrm{H}$ & 1.0 & -2.6715292693 & -0.2010342995 & 1.5224588773 \\
\hline C & 6.0 & 2.5714522585 & 0.6856361747 & 2.3140115006 \\
\hline C & 6.0 & 0.1780918359 & 0.2483216762 & 2.4207942574 \\
\hline $\mathrm{C}$ & 6.0 & 1.2512333276 & 0.7968384129 & 0.3369316341 \\
\hline C & 6.0 & 2.4985593904 & 0.9113093656 & 0.9382001412 \\
\hline C & 6.0 & 1.4195788695 & 0.3690581963 & 3.0462315152 \\
\hline $\mathrm{H}$ & 1.0 & -0.6998437543 & -0.0188880612 & 2.9963328010 \\
\hline S & 16.0 & 0.9374520600 & 1.0978902745 & -1.4014573582 \\
\hline $\mathrm{H}$ & 1.0 & 3.3806513095 & 1.1321752401 & 0.3513860627 \\
\hline $\mathrm{H}$ & 1.0 & 1.4964510298 & 0.2017585780 & 4.1129756127 \\
\hline $\mathrm{H}$ & 1.0 & 3.5276364023 & 0.7493741438 & 2.8166890589 \\
\hline $\mathrm{N}$ & 7.0 & 1.8762832193 & 0.2204175900 & -2.3782868421 \\
\hline S & 16.0 & 2.0668894544 & -1.3901765047 & -1.9416903410 \\
\hline C & 6.0 & 99181649 & -2.0910372000 & -3.5673895587 \\
\hline O & 8.0 & 0.8312831772 & -1.9119000588 & -1.3256030884 \\
\hline O & 8.0 & 3.3424441392 & -1.5827894139 & -1.2366889277 \\
\hline $\mathrm{H}$ & 1.0 & 3.0597576393 & -1.5909192382 & 38191735 \\
\hline $\mathrm{H}$ & 1.0 & 90037606 & 66713976 & 90123256 \\
\hline $\mathrm{H}$ & 1.0 & 2.4617046507 & -3.1471063676 & 51996751 \\
\hline & & 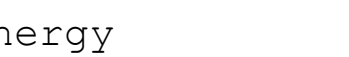 & -941447.7661 & $\mathrm{KCAL} / \mathrm{MOL}$ \\
\hline & ion & & 135.4656 & $\mathrm{KCAL} / \mathrm{MOL}$ \\
\hline & perat & re Correction & 146.061 & $\mathrm{KCAL} / \mathrm{MOL}$ \\
\hline & $3 d f, 2$ & $\omega$ & 47.6661 & IOL \\
\hline
\end{tabular}




\begin{tabular}{|c|c|c|c|c|c|}
\hline ATOI & & MULL. POP. & CHARGE & LOW. POP. & CHARGE \\
\hline & $\mathrm{H}$ & 0.853209 & 0.146791 & 0.875089 & 0.124911 \\
\hline 2 & C & 6.119459 & -0.119459 & 6.096354 & -0.096354 \\
\hline & C & 6.094857 & -0.094857 & 6.095448 & -0.095448 \\
\hline 4 & $\mathrm{C}$ & 6.105323 & -0.105323 & 6.097138 & -0.097138 \\
\hline 5 & C & 6.228518 & -0.228518 & 6.154291 & -0.154291 \\
\hline 6 & C & 5.938537 & 0.061463 & 6.002668 & -0.002668 \\
\hline 7 & C & 6.129547 & -0.129547 & 6.097449 & -0.097449 \\
\hline 8 & $\mathrm{H}$ & 0.865038 & 0.134962 & 0.878413 & 0.121587 \\
\hline 9 & C & 5.916444 & 0.083556 & 6.005050 & -0.005050 \\
\hline 10 & $\mathrm{H}$ & 0.865771 & 0.134229 & 0.881875 & 0.118125 \\
\hline 11 & $\mathrm{H}$ & 0.865206 & 0.134794 & 0.882532 & 0.117468 \\
\hline 12 & $\mathrm{C}$ & 6.103832 & -0.103832 & 6.089469 & -0.089469 \\
\hline 13 & $\mathrm{C}$ & 6.093900 & -0.093900 & 6.096593 & -0.096593 \\
\hline 14 & $\mathrm{C}$ & 6.290879 & -0.290879 & 6.165927 & -0.165927 \\
\hline 15 & C & 6.093965 & -0.093965 & 6.088587 & -0.088587 \\
\hline 16 & C & 6.130204 & -0.130204 & 6.096513 & -0.096513 \\
\hline 17 & $\mathrm{H}$ & 0.864886 & 0.135114 & 0.880038 & 0.119962 \\
\hline 18 & S & 15.192520 & 0.807480 & 15.188968 & 0.811032 \\
\hline 19 & $\mathrm{H}$ & 0.833231 & 0.166769 & 0.863515 & 0.136485 \\
\hline 20 & $\mathrm{H}$ & 0.865322 & 0.134678 & 0.882793 & 0.117207 \\
\hline 21 & $\mathrm{H}$ & 0.860517 & 0.139483 & 0.877343 & 0.122657 \\
\hline 22 & $\mathrm{~N}$ & 7.762419 & -0.762419 & 7.755241 & -0.755241 \\
\hline 23 & S & 14.767049 & 1.232951 & 14.679605 & 1.320395 \\
\hline 24 & C & 6.529079 & -0.529079 & 6.447809 & -0.447809 \\
\hline 25 & O & 8.578251 & -0.578251 & 8.625657 & -0.625657 \\
\hline 26 & O & 8.548220 & -0.548220 & 8.612188 & -0.612188 \\
\hline 27 & $\mathrm{H}$ & 0.828385 & 0.171615 & 0.856482 & 0.143518 \\
\hline 28 & $\mathrm{H}$ & 0.842133 & 0.157867 & 0.864621 & 0.135379 \\
\hline 29 & $\mathrm{H}$ & 0.833300 & 0.166700 & 0.862346 & 0.137654 \\
\hline
\end{tabular}




\section{C-carbomethoxycarbonyl-S, S-dimethylsulfonium ylide (39)}

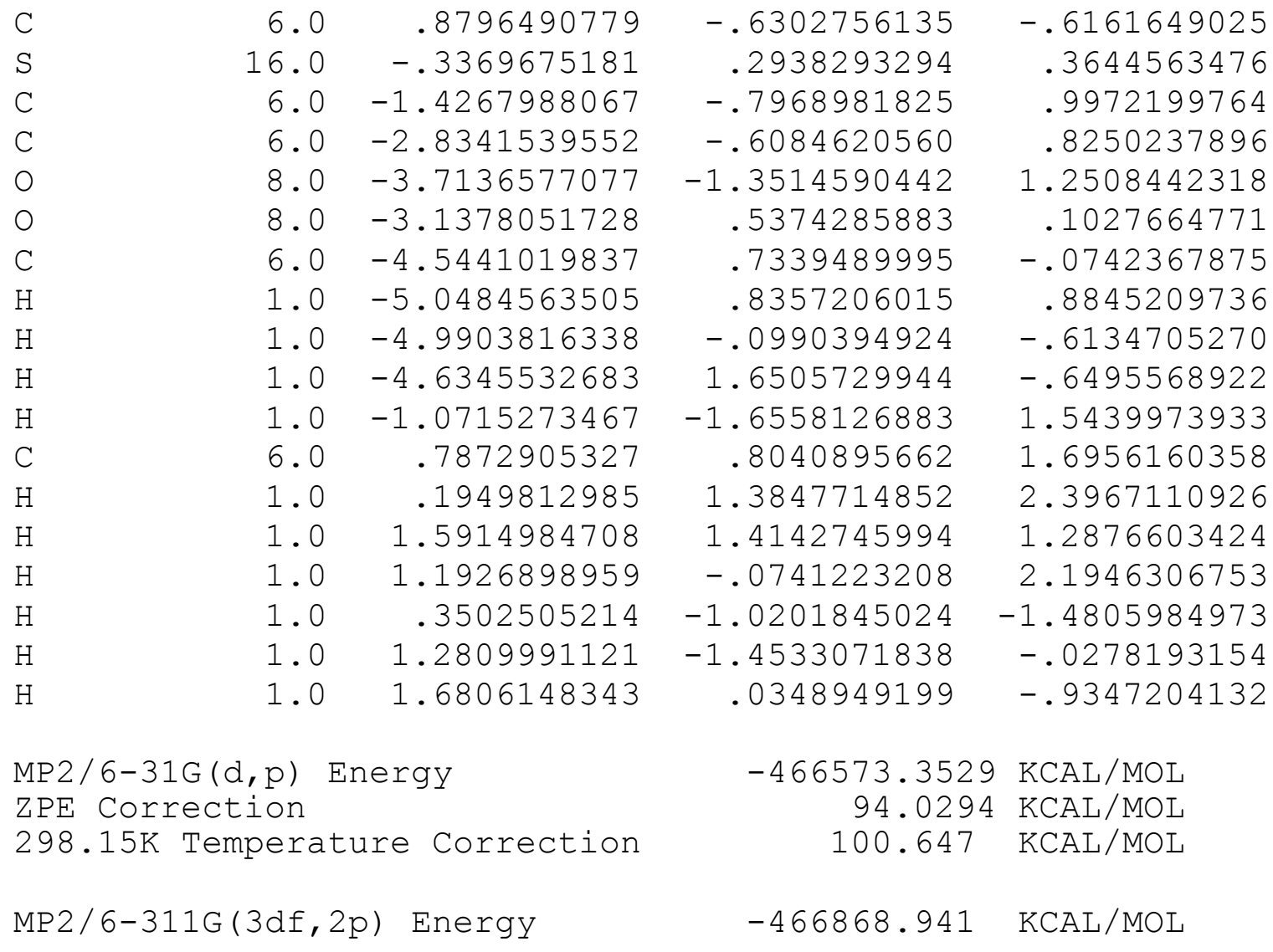

\begin{tabular}{|c|c|c|c|c|c|}
\hline ATOI & & MULL . POP . & CHARGE & LOW . POP. & CHARGE \\
\hline 1 & C & 6.483744 & -.483744 & 6.431270 & -.431270 \\
\hline 2 & S & 15.445206 & .554794 & 15.286359 & .713641 \\
\hline 3 & $\mathrm{C}$ & 6.530958 & -.530958 & 6.549389 & -.549389 \\
\hline 4 & C & 5.364054 & .635946 & 5.799662 & .200338 \\
\hline 5 & O & 8.516547 & -.516547 & 8.318860 & -.318860 \\
\hline 6 & O & 8.538632 & -.538632 & 8.259489 & -.259489 \\
\hline 7 & $\mathrm{C}$ & 6.061593 & -.061593 & 6.131422 & -.131422 \\
\hline 8 & $\mathrm{H}$ & .880049 & .119951 & .897713 & .102287 \\
\hline 9 & $\mathrm{H}$ & .879815 & .120185 & .896060 & .103940 \\
\hline 10 & $\mathrm{H}$ & .891654 & .108346 & .898048 & .101952 \\
\hline 11 & $\mathrm{H}$ & .865584 & .134416 & .880125 & .119875 \\
\hline 12 & $\mathrm{C}$ & 6.483760 & -.483760 & 6.431908 & -.431908 \\
\hline 13 & $\mathrm{H}$ & .827212 & .172788 & .859433 & .140567 \\
\hline 14 & $\mathrm{H}$ & .856377 & .143623 & .874230 & .125770 \\
\hline 15 & $\mathrm{H}$ & .845635 & .154365 & .875896 & .124104 \\
\hline 16 & $\mathrm{H}$ & .827195 & .172805 & .860272 & .139728 \\
\hline 17 & $\mathrm{H}$ & .845605 & .154395 & .875400 & .124600 \\
\hline 18 & $\mathrm{H}$ & .856380 & .143620 & .874464 & .125536 \\
\hline
\end{tabular}




\section{C-carbomethoxycarbonyl thiophenesulfonium ylide (40)}

$\begin{array}{lrr}\mathrm{C} & 6.0 & -.3826843357 \\ \mathrm{~S} & 16.0 & -.2695554129 \\ \mathrm{C} & 6.0 & -1.7679149566 \\ \mathrm{C} & 6.0 & -1.9798660315 \\ \mathrm{C} & 6.0 & -1.0115242218 \\ \mathrm{C} & 6.0 & -.0881235944 \\ \mathrm{H} & 1.0 & .7456294214 \\ \mathrm{H} & 1.0 & -1.0324052266 \\ \mathrm{H} & 1.0 & -2.7868159689 \\ \mathrm{H} & 1.0 & -2.3120487254 \\ \mathrm{C} & 6.0 & .4926633858 \\ \mathrm{O} & 8.0 & 1.3672217941 \\ \mathrm{C} & 6.0 & 2.2436262178 \\ \mathrm{H} & 1.0 & 2.8634140124 \\ \mathrm{H} & 1.0 & 2.8578553088 \\ \mathrm{H} & 1.0 & 1.6785374982 \\ \mathrm{O} & 8.0 & .4924603827 \\ \mathrm{H} & 1.0 & -1.1104795471\end{array}$

$\begin{array}{rr}.7460177610 & -.8291535984 \\ -.0228255176 & .6425759102 \\ -.6951639822 & 1.2892422593 \\ -.2704988224 & 2.5584319521 \\ .7180451246 & 3.0000005132 \\ 1.0194962932 & 2.0552749020 \\ 1.7015578373 & 2.0993791176 \\ 1.1656545900 & 3.9833507043 \\ -.6255677624 & 3.1833441618 \\ -1.4197079719 & .7050663233 \\ .3670874425 & -1.9013709844 \\ -.6465642860 & -1.5517636164 \\ -1.0288409956 & -2.6185653903 \\ -1.8202907515 & -2.2086739133 \\ -.1892214126 & -2.9366235153 \\ -1.3912143539 & -3.4745842906 \\ .8674770782 & -3.0205778368 \\ 1.5245597290 & -.9753626985\end{array}$

MP2 / 6-31G (d,p) Energy

ZPE Correction

298.15K Temperature Correction

MP2/6-311G (3df,2p) Energy

$\begin{array}{cc}-513483.2346 & \mathrm{KCAL} / \mathrm{MOL} \\ 86.6304 & \mathrm{KCAL} / \mathrm{MOL} \\ 93.72 & \mathrm{KCAL} / \mathrm{MOL} \\ -513827.9102 & \mathrm{KCAL} / \mathrm{MOL}\end{array}$

\begin{tabular}{|c|c|c|c|c|c|}
\hline ATO & & MULL. POP. & CHARGE & LOW. POP. & CHARGE \\
\hline & C & 6.509939 & -.509939 & 6.522317 & -.522317 \\
\hline 2 & S & 15.372382 & .627618 & 15.237128 & .762872 \\
\hline 3 & C & 6.331538 & -.331538 & 6.269417 & -.269417 \\
\hline 4 & $\mathrm{C}$ & 6.080960 & -.080960 & 6.107306 & -.107306 \\
\hline 5 & $\mathrm{C}$ & 6.080953 & -.080953 & 6.105464 & -.105464 \\
\hline 6 & C & 6.331542 & -.331542 & 6.267321 & -.267321 \\
\hline 7 & $\mathrm{H}$ & .820574 & .179426 & .853354 & .146646 \\
\hline 8 & $\mathrm{H}$ & .846041 & .153959 & .870744 & .129256 \\
\hline 9 & $\mathrm{H}$ & .846038 & .153962 & .868786 & .131214 \\
\hline 10 & $\mathrm{H}$ & .820591 & .179409 & .852158 & .147842 \\
\hline 11 & C & 5.366853 & .633147 & 5.801231 & .198769 \\
\hline 12 & O & 8.534188 & -.534188 & 8.252391 & -.252391 \\
\hline 13 & C & 6.064793 & -.064793 & 6.132990 & -.132990 \\
\hline 14 & $\mathrm{H}$ & .887261 & .112739 & .894603 & .105397 \\
\hline 15 & $\mathrm{H}$ & .876894 & .123106 & .894913 & .105087 \\
\hline 16 & $\mathrm{H}$ & .876863 & .123137 & .895022 & .104978 \\
\hline 17 & O & 8.506800 & -.506800 & 8.306352 & -.306352 \\
\hline 18 & $\mathrm{H}$ & .845790 & .154210 & .868502 & .131498 \\
\hline
\end{tabular}




\section{C-carbomethoxycarbonyl dibenzothiophenesulfonium ylide (41)}

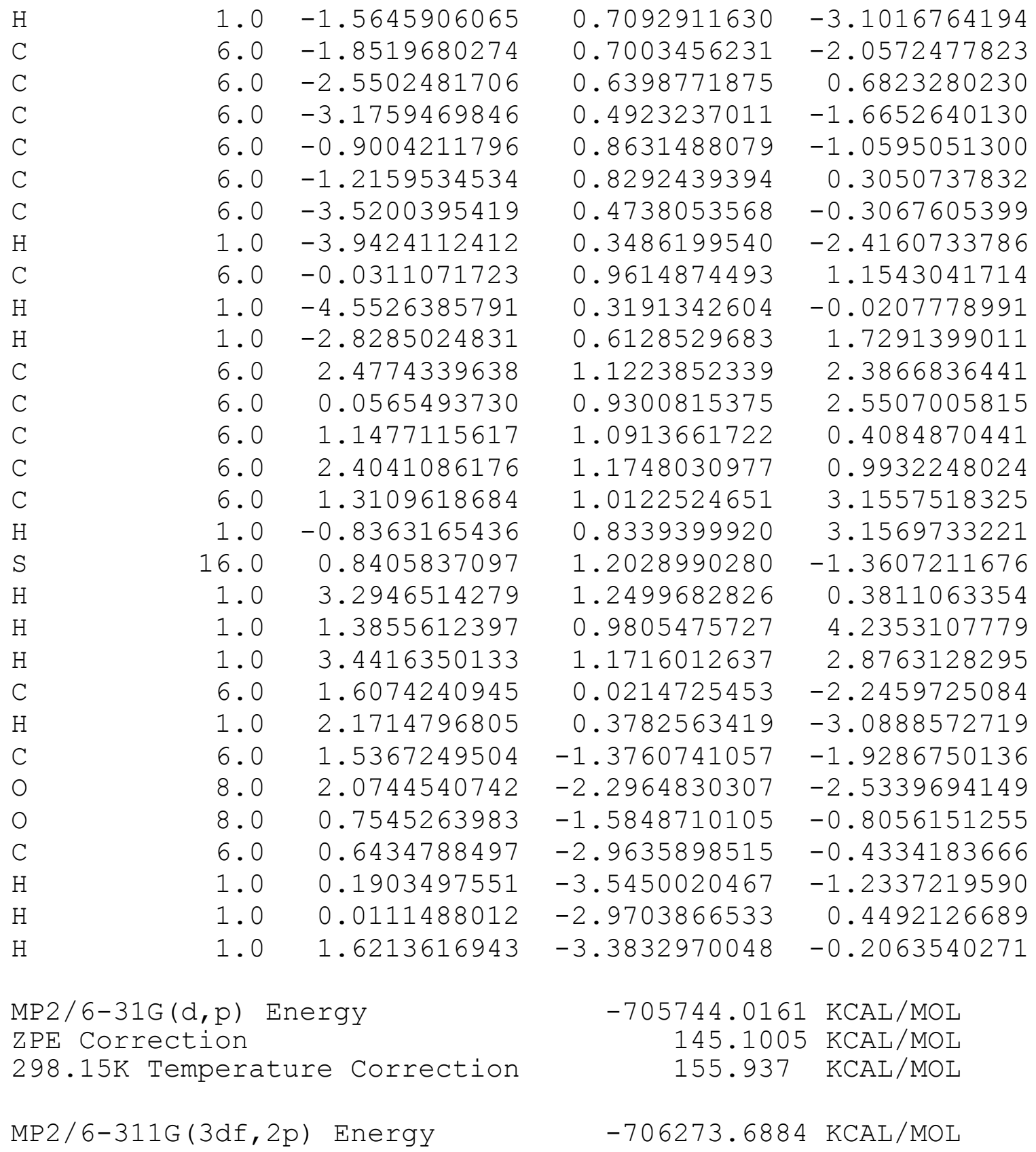




\begin{tabular}{|c|c|c|c|c|c|}
\hline ATOI & & MULL . POP . & CHARGE & LOW. POP. & CHARGE \\
\hline 1 & $\mathrm{H}$ & 0.851158 & 0.148842 & 0.874444 & 0.125556 \\
\hline 2 & $\mathrm{C}$ & 6.121811 & -0.121811 & 6.097440 & -0.097440 \\
\hline 3 & C & 6.099999 & -0.099999 & 6.100257 & -0.100257 \\
\hline 4 & C & 6.107585 & -0.107585 & 6.099418 & -0.099418 \\
\hline 5 & C & 6.225399 & -0.225399 & 6.150736 & -0.150736 \\
\hline 6 & $\mathrm{C}$ & 5.941808 & 0.058192 & 6.009511 & -0.009511 \\
\hline 7 & $\mathrm{C}$ & 6.132247 & -0.132247 & 6.102333 & -0.102333 \\
\hline 8 & $\mathrm{H}$ & 0.865645 & 0.134355 & 0.878868 & 0.121132 \\
\hline 9 & C & 5.941906 & 0.058094 & 6.009189 & -0.009189 \\
\hline 10 & $\mathrm{H}$ & 0.868227 & 0.131773 & 0.883392 & 0.116608 \\
\hline 11 & $\mathrm{H}$ & 0.868512 & 0.131488 & 0.884671 & 0.115329 \\
\hline 12 & C & 6.107587 & -0.107587 & 6.097847 & -0.097847 \\
\hline 13 & C & 6.099999 & -0.099999 & 6.103121 & -0.103121 \\
\hline 14 & C & 6.225365 & -0.225365 & 6.150136 & -0.150136 \\
\hline 15 & $\mathrm{C}$ & 6.121797 & -0.121797 & 6.100125 & -0.100125 \\
\hline 16 & $\mathrm{C}$ & 6.132252 & -0.132252 & 6.101008 & -0.101008 \\
\hline 17 & $\mathrm{H}$ & 0.868508 & 0.131492 & 0.882207 & 0.117793 \\
\hline 18 & S & 15.364044 & 0.635956 & 15.282411 & 0.717589 \\
\hline 19 & $\mathrm{H}$ & 0.851127 & 0.148873 & 0.872070 & 0.127930 \\
\hline 20 & $\mathrm{H}$ & 0.868235 & 0.131765 & 0.884538 & 0.115462 \\
\hline 21 & $\mathrm{H}$ & 0.865642 & 0.134358 & 0.880310 & 0.119690 \\
\hline 22 & C & 6.524120 & -0.524120 & 6.527860 & -0.527860 \\
\hline 23 & $\mathrm{H}$ & 0.844455 & 0.155545 & 0.860245 & 0.139755 \\
\hline 24 & $\mathrm{C}$ & 5.359418 & 0.640582 & 5.801730 & 0.198270 \\
\hline 25 & O & 8.499241 & -0.499241 & 8.301099 & -0.301099 \\
\hline 26 & O & 8.540004 & -0.540004 & 8.249149 & -0.249149 \\
\hline 27 & $\mathrm{C}$ & 6.063807 & -0.063807 & 6.130796 & -0.130796 \\
\hline 28 & $\mathrm{H}$ & 0.876269 & 0.123731 & 0.893751 & 0.106249 \\
\hline 29 & $\mathrm{H}$ & 0.887489 & 0.112511 & 0.895528 & 0.104472 \\
\hline 30 & $\mathrm{H}$ & 0.876348 & 0.123652 & 0.895812 & 0.104188 \\
\hline
\end{tabular}




\section{C-dicarbomethoxycarbonyl-S, S-dimethylsulfonium ylide (42)}

\begin{tabular}{|c|c|c|c|c|}
\hline$S$ & 16.0 & -0.7666248948 & -1.6048660991 & -0.9662789832 \\
\hline C & 6.0 & -0.0603509566 & -0.6290787874 & 0.2624784976 \\
\hline C & 6.0 & -0.8373300053 & 0.1406994390 & 1.1975853341 \\
\hline C & 6.0 & 1.2326829787 & -1.2175640657 & 0.6353210791 \\
\hline C & 6.0 & -1.9809349644 & -2.7272538714 & -0.2354876758 \\
\hline C & 6.0 & -1.8329512112 & -0.5334087296 & -1.9448448740 \\
\hline O & 8.0 & 1.5021787794 & -2.3975369319 & 0.4063868470 \\
\hline O & 8.0 & 2.1678545570 & -0.4515742759 & 1.2477881658 \\
\hline O & 8.0 & -2.1886644548 & 0.1781700226 & 0.8610369534 \\
\hline 0 & 8.0 & -0.4294421100 & 0.7169065474 & 2.2010063960 \\
\hline C & 6.0 & 2.1655738100 & 0.9756985485 & 1.0922595186 \\
\hline C & 6.0 & -2.9786208727 & 0.9414267550 & 1.7883874069 \\
\hline $\mathrm{H}$ & 1 . & 1.8281831410 & 1.4432401733 & 2.0100259374 \\
\hline $\mathrm{H}$ & 1 . & 1.5167229674 & 1.2729006126 & 0.2712873925 \\
\hline $\mathrm{H}$ & 1 . & 3.1947263338 & 1.2513942920 & 0.8731499397 \\
\hline $\mathrm{H}$ & 1 . & -3.9946720620 & 0.8852128734 & 1.4098447211 \\
\hline $\mathrm{H}$ & 1 . & -2.6394792553 & 1.9737732412 & 1.823846449 \\
\hline $\mathrm{H}$ & 1 . & -2.9174779783 & 0.5171029575 & 2.7874704533 \\
\hline $\mathrm{H}$ & 1 . & -1.4044134603 & -3.4025973113 & 0.390982977 \\
\hline $\mathrm{H}$ & & -2.4741470419 & -3.2830687740 & -1.0307658302 \\
\hline $\mathrm{H}$ & & -2.6925116537 & -2.1569148451 & 0.3532841964 \\
\hline $\mathrm{H}$ & & -1.1913906229 & 0.2374466880 & -2.362543753 \\
\hline $\mathrm{H}$ & & -2.6044887595 & -0.0912054300 & -1.326461056 \\
\hline & & -2.2550206732 & -1.1364099194 & -2.746231452 \\
\hline \multirow{2}{*}{\multicolumn{3}{|c|}{ MP2 / 6-31G $(\mathrm{d}, \mathrm{p})$ Energy }} & -609188.1826 & $\mathrm{KCAL} / \mathrm{MOL}$ \\
\hline & & & 121.9832 & $\mathrm{KCAL} / \mathrm{MOL}$ \\
\hline \multicolumn{3}{|c|}{ 298.15K Temperature Correction } & 131.749 & KCAL/MOL \\
\hline \multicolumn{3}{|c|}{ MP2/6-311G (3df,2p) Energy } & -609648.4771 & $\mathrm{KCAL} / \mathrm{MOL}$ \\
\hline
\end{tabular}




\begin{tabular}{rrrrrr}
\multicolumn{1}{l}{ TOTAL MULLIKEN AND LOWDIN ATOMIC } & POPULATIONS \\
ATOM & MULL.POP & CHARGE & \multicolumn{1}{c}{ LOW.POP. } & \multicolumn{1}{c}{ CHARGE } \\
$1 \mathrm{~S}$ & 15.386457 & 0.613543 & 15.268977 & 0.731023 \\
2 & $\mathrm{C}$ & 6.542455 & -0.542455 & 6.444724 & -0.444724 \\
3 & $\mathrm{C}$ & 5.347274 & 0.652726 & 5.804434 & 0.195566 \\
4 & $\mathrm{C}$ & 5.371609 & 0.628391 & 5.803549 & 0.196451 \\
5 & $\mathrm{C}$ & 6.504166 & -0.504166 & 6.423388 & -0.423388 \\
6 & $\mathrm{C}$ & 6.497520 & -0.497520 & 6.416429 & -0.416429 \\
7 & $\mathrm{O}$ & 8.472162 & -0.472162 & 8.297814 & -0.297814 \\
8 & $\mathrm{O}$ & 8.484830 & -0.484830 & 8.197585 & -0.197585 \\
9 & $\mathrm{O}$ & 8.560322 & -0.560322 & 8.260959 & -0.260959 \\
10 & $\mathrm{O}$ & 8.489294 & -0.489294 & 8.292240 & -0.292240 \\
11 & $\mathrm{C}$ & 6.067001 & -0.067001 & 6.127354 & -0.127354 \\
12 & $\mathrm{C}$ & 6.069792 & -0.069792 & 6.127843 & -0.127843 \\
13 & $\mathrm{H}$ & 0.851758 & 0.148242 & 0.884792 & 0.115208 \\
14 & $\mathrm{H}$ & 0.890962 & 0.109038 & 0.897825 & 0.102175 \\
15 & $\mathrm{H}$ & 0.883906 & 0.116094 & 0.898885 & 0.101115 \\
16 & $\mathrm{H}$ & 0.886545 & 0.113455 & 0.897107 & 0.102893 \\
17 & $\mathrm{H}$ & 0.868343 & 0.131657 & 0.893378 & 0.106622 \\
18 & $\mathrm{H}$ & 0.865757 & 0.134243 & 0.891566 & 0.108434 \\
19 & $\mathrm{H}$ & 0.805452 & 0.194548 & 0.848571 & 0.151429 \\
20 & $\mathrm{H}$ & 0.850712 & 0.149288 & 0.869833 & 0.130167 \\
21 & $\mathrm{H}$ & 0.819684 & 0.180316 & 0.864637 & 0.135363 \\
22 & $\mathrm{H}$ & 0.826337 & 0.173663 & 0.857133 & 0.142867 \\
$23 \mathrm{H}$ & 0.809350 & 0.190650 & 0.861177 & 0.138823 \\
24 & $\mathrm{H}$ & 0.848313 & 0.151687 & 0.869801 & 0.130199
\end{tabular}


Computed BDEs as a function of basis set

Table S1. Computational and experimental BDEs for sulfoxides (1-4) in $\mathrm{kcal} / \mathrm{mol}$.

\begin{tabular}{|c|c|c|c|c|}
\hline Method $^{\mathrm{a}}$ & 1 & 2 & 3 & 4 \\
\hline MP2/6-311++G(3df,2p) & 89.6 & 88.7 & 87.4 & 85.1 \\
\hline MP2/6-311G(3df,2p) & 88.1 & 87.8 & 86.5 & 85.3 \\
\hline MP2/6-311++G(d) & 67.9 & 67.4 & 66.2 & 63.1 \\
\hline $\mathrm{MP} 2 / 6-31+\mathrm{G}(2 \mathrm{~d}, \mathrm{p})$ & 78.4 & 78.0 & 76.9 & 74.7 \\
\hline MP2/6-31G(2d) & 73.4 & 73.9 & 72.7 & 72.1 \\
\hline MP2/6-31G(d,p) & 67.3 & 67.6 & 66.7 & 66.0 \\
\hline MP2/6-311G(d) & 64.3 & 65.0 & 63.9 & 62.7 \\
\hline MP2/6-31+G(d) & 72.6 & 72.0 & 71.0 & 68.2 \\
\hline MP2/6-31G(d) & 67.7 & 68.0 & 67.0 & 66.1 \\
\hline B3LYP/6-311++G(3df,2p) & 81.9 & 80.8 & 79.9 & 77.7 \\
\hline B3LYP/6-311G(3df,2p) & 81.9 & 81.3 & 80.5 & 79.2 \\
\hline B3LYP/6-311++G(d) & 67.2 & 66.8 & 66.0 & 64.1 \\
\hline B3LYP/6-31+G(2d,p) & 75.1 & 74.1 & 73.3 & 71.2 \\
\hline B3LYP/6-31G(2d) & 72.1 & 72.0 & 71.3 & 70.7 \\
\hline B3LYP/6-31G(d,p) & 67.9 & 68.1 & 67.3 & 66.8 \\
\hline B3LYP/6-311G(d) & 65.6 & 65.3 & 65.6 & 65.0 \\
\hline B3LYP/6-31+G(d) & 69.3 & 68.6 & 67.9 & 65.8 \\
\hline B3LYP/6-31G(d) & 67.9 & 68.0 & 67.3 & 66.6 \\
\hline G3 $(0 \mathrm{~K})$ & 84.3 & 84.0 & 83.0 & N/A \\
\hline G3 (298 K) & 82.3 & 81.8 & 80.8 & N/A \\
\hline $\operatorname{Expt}^{\mathrm{b}}$ & $86.5 \pm 0.5$ & $88.8 \pm 0.6$ & $88.7 \pm 1.2$ & $89.3 \pm 1.4$ \\
\hline
\end{tabular}


Table S2. Computational and experimental BDEs for sulfoxides (5-9) in $\mathrm{kcal} / \mathrm{mol}$.

\begin{tabular}{lccccc}
\hline \multicolumn{1}{c}{ Method $^{\text {a }}$} & $\mathbf{5}$ & $\mathbf{6}$ & $\mathbf{7}$ & $\mathbf{8}$ & $\mathbf{9}$ \\
\hline MP2/6-311++G(3df,2p) & 89.3 & 86.0 & 60.4 & 67.3 & 72.1 \\
MP2/6-311G(3df,2p) & 86.5 & 85.5 & 59.6 & 66.4 & 71.3 \\
MP2/6-311++G(d) & 66.1 & 65.3 & 40.1 & & \\
MP2/6-31+G(2d,p) & 75.1 & 75.4 & 50.3 & & \\
MP2/6-31G(2d) & 70.5 & 71.9 & 47.5 & & \\
MP2/6-31G(d,p) & 65.8 & 65.9 & 42.3 & & \\
MP2/6-311G(d) & 62.5 & 62.8 & 36.6 & & \\
MP2/6-31+G(d) & 70.6 & 70.0 & 45.1 & & \\
MP2/6-31G(d) & 66.1 & 66.3 & 42.6 & & \\
B3LYP/6-311++G(3df,2p) & 80.7 & 77.8 & 56.4 & & \\
B3LYP/6-311G(3df,2p) & 80.9 & 78.7 & 57.0 & & \\
B3LYP/6-311++G(d) & 66.7 & 63.9 & 42.9 & & \\
B3LYP/6-31+G(2d,p) & 73.8 & 71.3 & 50.1 & & N/A \\
B3LYP/6-31G(2d) & 71.3 & 69.8 & 49.4 & & \\
B3LYP/6-31G(d,p) & 67.3 & 65.6 & 46.0 & & \\
B3LYP/6-311G(d) & 65.2 & 63.6 & 41.8 & & \\
B3LYP/6-31+G(d) & 68.7 & 65.9 & 45.2 & & \\
B3LYP/6-31G(d) & 67.3 & 65.7 & 46.1 & & \\
G3 (0 K) & 80.8 & 81.0 & 58.5 & N/A \\
G3 (298 K) & 79.1 & 79.0 & 56.5 & N/A \\
a All BDE were determined from the listed method energies performed at the MP2/6-31G(d,p) \\
geometry and include the unscaled ZPE and a temperature correction to 298.15 K. & \\
& & & & \\
\end{tabular}


Table S3. BDEs for 1-4 from isodesmic reactions at different basis sets, in kcal $/ \mathrm{mol}$.

\begin{tabular}{|c|c|c|c|c|}
\hline Method $^{\mathrm{a}}$ & $\mathbf{1}$ & 2 & 3 & 4 \\
\hline \multirow[t]{4}{*}{$\mathrm{MP} 2 / 6-311++\mathrm{G}(3 \mathrm{df}, 2 \mathrm{p})$} & N/A & 85.6 & 84.2 & 86.1 \\
\hline & $(89.8)$ & (N/A) & $(87.6)$ & $(85.2)$ \\
\hline & $\{91.0\}$ & $\{90.0\}$ & $\{\mathrm{N} / \mathrm{A}\}$ & $\{86.4\}$ \\
\hline & [93.9] & [93.0] & [91.6] & {$[\mathrm{N} / \mathrm{A}]$} \\
\hline \multirow[t]{4}{*}{ MP2/6-311G(3df,2p) } & N/A & 86.2 & 84.9 & 84.9 \\
\hline & $(89.2)$ & (N/A) & $(87.6)$ & $(86.4)$ \\
\hline & $\{90.3\}$ & $\{90.0\}$ & $\{\mathrm{N} / \mathrm{A}\}$ & $\{87.5\}$ \\
\hline & [92.1] & [91.8] & [90.5] & {$[\mathrm{N} / \mathrm{A}]$} \\
\hline \multirow[t]{4}{*}{ MP2/6-311++G(d) } & $\mathrm{N} / \mathrm{A}$ & 86.0 & 84.8 & 84.7 \\
\hline & $(89.4)$ & (N/A) & $(87.7)$ & $(84.6)$ \\
\hline & $\{90.4\}$ & $\{89.9\}$ & $\{\mathrm{N} / \mathrm{A}\}$ & $\{85.6\}$ \\
\hline & [94.1] & [93.6] & [92.4] & {$[\mathrm{N} / \mathrm{A}]$} \\
\hline \multirow[t]{4}{*}{ MP2/6-31+G(2d,p) } & $\mathrm{N} / \mathrm{A}$ & 86.1 & 84.9 & 83.1 \\
\hline & $(89.3)$ & $(\mathrm{N} / \mathrm{A})$ & $(87.7)$ & $(85.5)$ \\
\hline & $\{90.3\}$ & $\{89.9\}$ & $\{\mathrm{N} / \mathrm{A}\}$ & $\{86.5\}$ \\
\hline & [93.1] & [92.7] & [91.5] & {$[\mathrm{N} / \mathrm{A}]$} \\
\hline \multirow[t]{4}{*}{ MP2/6-31G(2d) } & N/A & 86.9 & 85.8 & 83.6 \\
\hline & $(88.5)$ & $(\mathrm{N} / \mathrm{A})$ & $(87.8)$ & $(87.2)$ \\
\hline & $\{89.4\}$ & $\{89.8\}$ & $\{\mathrm{N} / \mathrm{A}\}$ & $\{88.1\}$ \\
\hline & [90.6] & [91.0] & [89.9] & {$[\mathrm{N} / \mathrm{A}]$} \\
\hline \multirow[t]{4}{*}{ MP2/6-31G(d,p) } & N/A & 86.8 & 85.9 & 85.0 \\
\hline & (88.6) & $(\mathrm{N} / \mathrm{A})$ & $(88.0)$ & $(87.2)$ \\
\hline & $\{89.3\}$ & $\{89.6\}$ & $\{\mathrm{N} / \mathrm{A}\}$ & $\{87.9\}$ \\
\hline & [90.7] & [91.0] & [90.1] & {$[\mathrm{N} / \mathrm{A}]$} \\
\hline \multirow[t]{4}{*}{ MP2/6-311G(d) } & $\mathrm{N} / \mathrm{A}$ & 87.1 & 86.0 & 84.6 \\
\hline & $(88.3)$ & (N/A) & $(87.8)$ & $(86.6)$ \\
\hline & $\{89.2\}$ & $\{89.8\}$ & $\{86.6\}$ & $\{87.5\}$ \\
\hline & [91.0] & [91.6] & [90.5] & {$[\mathrm{N} / \mathrm{A}]$} \\
\hline \multirow[t]{4}{*}{$\mathrm{MP} 2 / 6-31+\mathrm{G}(\mathrm{d})$} & $\mathrm{N} / \mathrm{A}$ & 85.9 & 84.9 & 84.5 \\
\hline & (89.5) & (N/A) & $(87.9)$ & $(85.1)$ \\
\hline & $\{90.3\}$ & $\{89.7\}$ & $\{\mathrm{N} / \mathrm{A}\}$ & $\{85.9\}$ \\
\hline & [93.7] & [93.1] & [92.1] & {$[\mathrm{N} / \mathrm{A}]$} \\
\hline \multirow[t]{4}{*}{ MP2/6-31G(d) } & $\mathrm{N} / \mathrm{A}$ & 86.8 & 85.8 & 84.9 \\
\hline & (88.6) & $(\mathrm{N} / \mathrm{A})$ & $(87.9)$ & $(87.1)$ \\
\hline & $\{89.4\}$ & $\{89.7\}$ & $\{\mathrm{N} / \mathrm{A}\}$ & $\{87.8\}$ \\
\hline & [90.9] & [91.1] & [90.2] & {$[\mathrm{N} / \mathrm{A}]$} \\
\hline \multirow[t]{4}{*}{ B3LYP/6-311++G(3df,2p) } & $\mathrm{N} / \mathrm{A}$ & 84.2 & 84.4 & 82.3 \\
\hline & $(90.0)$ & (N/A) & $(88.0)$ & $(85.8)$ \\
\hline & $\{90.8\}$ & $\{89.6\}$ & $\{\mathrm{N} / \mathrm{A}\}$ & $\{86.5\}$ \\
\hline & [93.5] & [92.4] & [91.5] & {$[\mathrm{N} / \mathrm{A}]$} \\
\hline \multirow[t]{3}{*}{ B3LYP/6-311G(3df,2p) } & $\mathrm{N} / \mathrm{A}$ & 84.9 & 85.1 & 83.8 \\
\hline & $(89.5)$ & $(\mathrm{N} / \mathrm{A})$ & $(88.1)$ & $(86.8)$ \\
\hline & $\{90.1\}$ & $\{89.5\}$ & $\{\mathrm{N} / \mathrm{A}\}$ & $\{87.4\}$ \\
\hline
\end{tabular}




\begin{tabular}{|c|c|c|c|c|}
\hline & {$[92.0]$} & {$[91.4]$} & [90.6] & {$[\mathrm{N} / \mathrm{A}]$} \\
\hline \multirow[t]{4}{*}{ B3LYP/6-311++G(d) } & $\mathrm{N} / \mathrm{A}$ & 84.8 & 85.3 & 83.4 \\
\hline & $(89.4)$ & (N/A) & $(88.2)$ & $(86.3)$ \\
\hline & $\{89.9\}$ & $\{89.4\}$ & $\{\mathrm{N} / \mathrm{A}\}$ & $\{86.8\}$ \\
\hline & {$[92.4]$} & [91.9] & [91.2] & {$[\mathrm{N} / \mathrm{A}]$} \\
\hline \multirow[t]{4}{*}{$\mathrm{B} 3 \mathrm{LYP} / 6-31+\mathrm{G}(2 \mathrm{~d}, \mathrm{p})$} & $\mathrm{N} / \mathrm{A}$ & 84.9 & 84.7 & 82.6 \\
\hline & (89.9) & (N/A) & $(88.1)$ & $(86.0)$ \\
\hline & $\{90.5\}$ & $\{89.5\}$ & $\{\mathrm{N} / \mathrm{A}\}$ & $\{86.6\}$ \\
\hline & [93.2] & [92.2] & [91.4] & {$[\mathrm{N} / \mathrm{A}]$} \\
\hline \multirow[t]{4}{*}{ B3LYP/6-31G(2d) } & $\mathrm{N} / \mathrm{A}$ & 85.8 & 85.7 & 85.1 \\
\hline & $(89.0)$ & $(\mathrm{N} / \mathrm{A})$ & $(88.2)$ & $(87.6)$ \\
\hline & $\{89.5\}$ & $\{89.4\}$ & $\{\mathrm{N} / \mathrm{A}\}$ & $\{88.1\}$ \\
\hline & {$[90.7]$} & [90.6] & [89.9] & {$[\mathrm{N} / \mathrm{A}]$} \\
\hline \multirow[t]{4}{*}{ B3LYP/6-31G(d,p) } & $\mathrm{N} / \mathrm{A}$ & 85.9 & 85.9 & 85.3 \\
\hline & $(88.8)$ & (N/A) & $(88.2)$ & $(87.6)$ \\
\hline & $\{89.3\}$ & $\{89.4\}$ & $\{\mathrm{N} / \mathrm{A}\}$ & $\{88.1\}$ \\
\hline & {$[90.5]$} & {$[90.6]$} & [89.9] & {$[\mathrm{N} / \mathrm{A}]$} \\
\hline \multirow[t]{4}{*}{ B3LYP/6-311G(d) } & N/A & 86.0 & 86.5 & 85.8 \\
\hline & $(88.2)$ & (N/A) & $(88.2)$ & $(87.2)$ \\
\hline & $\{88.7\}$ & $\{89.4\}$ & $\{\mathrm{N} / \mathrm{A}\}$ & $\{88.0\}$ \\
\hline & [90.0] & [90.6] & {$[90.0]$} & [N/A] \\
\hline \multirow[t]{4}{*}{ B3LYP/6-31+G(d) } & $\mathrm{N} / \mathrm{A}$ & 84.9 & 85.0 & 83.0 \\
\hline & $(89.7)$ & (N/A) & $(88.2)$ & $(86.2)$ \\
\hline & $\{90.2\}$ & $\{89.4\}$ & $\{\mathrm{N} / \mathrm{A}\}$ & $\{86.6\}$ \\
\hline & [92.8] & [92.0] & [91.4] & {$[\mathrm{N} / \mathrm{A}]$} \\
\hline \multirow[t]{4}{*}{ B3LYP/6-31G(d) } & $\mathrm{N} / \mathrm{A}$ & 85.8 & 85.9 & 85.3 \\
\hline & $(88.8)$ & (N/A) & $(88.2)$ & $(87.6)$ \\
\hline & $\{89.3\}$ & $\{89.4\}$ & $\{\mathrm{N} / \mathrm{A}\}$ & $\{88.1\}$ \\
\hline & {$[90.5]$} & {$[90.6]$} & [89.9] & {$[\mathrm{N} / \mathrm{A}]$} \\
\hline \multirow[t]{4}{*}{ G3 (0 K) } & N/A & 86.2 & 85.2 & $\mathrm{~N} / \mathrm{A}$ \\
\hline & (89.2) & (N/A) & (87.9) & (N/A) \\
\hline & $\{90.0\}$ & $\{89.7\}$ & $\{\mathrm{N} / \mathrm{A}\}$ & $\{\mathrm{N} / \mathrm{A}\}$ \\
\hline & [N/A] & {$[\mathrm{N} / \mathrm{A}]$} & [N/A] & {$[\mathrm{N} / \mathrm{A}]$} \\
\hline \multirow[t]{4}{*}{ G3 (298 K) } & $\mathrm{N} / \mathrm{A}$ & 86.1 & 85.1 & $\mathrm{~N} / \mathrm{A}$ \\
\hline & (89.2) & (N/A) & (87.9) & $(\mathrm{N} / \mathrm{A})$ \\
\hline & $\{90.1\}$ & $\{89.7\}$ & $\{\mathrm{N} / \mathrm{A}\}$ & $\{\mathrm{N} / \mathrm{A}\}$ \\
\hline & [N/A] & [N/A] & {$[\mathrm{N} / \mathrm{A}]$} & {$[\mathrm{N} / \mathrm{A}]$} \\
\hline $\mathrm{Expt}^{\mathrm{b}}$ & $86.5 \pm 0.5$ & $88.8 \pm 0.6$ & $88.7 \pm 1.2$ & $89.3 \pm 1$ \\
\hline
\end{tabular}

${ }^{a}$ All BDE were determined from the listed method energies performed at the MP2/6-31G(d,p) geometry and include the unscaled ZPE and temperature correction to $298.15 \mathrm{~K}$. BDE were determined by four isodesmic reactions using $1: \mathrm{BDE}_{\text {std }}=86.5 \mathrm{kcal} / \mathrm{mol}, 2:\left(\mathrm{BDE}_{\mathrm{std}}=88.8\right.$ $\mathrm{kcal} / \mathrm{mol}), 3:\left\{\mathrm{BDE}_{\mathrm{std}}=88.7 \mathrm{kcal} / \mathrm{mol}\right\}$, and 4: $\left[\mathrm{BDE}_{\mathrm{std}}=89.3 \mathrm{kcal} / \mathrm{mol}\right]^{\mathrm{b}}$ Experimental BDE determined from $\Delta \mathrm{H}_{f}^{\circ}$ values from the NIST webbook. 
Table S4. BDEs for sulfilimines 10-15 as a function of basis set.

\begin{tabular}{lcccccc}
\hline \multicolumn{1}{c}{ Method $^{\mathrm{a}}$} & $\mathbf{1 0}$ & $\mathbf{1 1}$ & $\mathbf{1 2}$ & $\mathbf{1 3}$ & $\mathbf{1 4}$ & $\mathbf{1 5}$ \\
\hline MP2/6-311++G(3df,2p) & 47.5 & 25.5 & 43.1 & 34.2 & 38.3 & 42.4 \\
MP2/6-311G(3df,2p) & 46.2 & 23.3 & 41.2 & 33.2 & 37.0 & 41.5 \\
MP2/6-311++G(d) & 31.3 & 9.3 & 27.6 & 20.0 & 22.4 & 26.6 \\
MP2/6-31+G(2d,p) & 37.4 & 15.3 & 33.2 & 24.6 & 28.2 & 32.9 \\
MP2/6-31G(2d) & 32.9 & 11.4 & 28.2 & 21.0 & 24.4 & 29.1 \\
MP2/6-31G(d,p) & 27.1 & 5.2 & 23.6 & 15.3 & 18.8 & 23.4 \\
MP2/6-311G(d) & 28.7 & 5.9 & 24.4 & 14.3 & 20.4 & 24.9 \\
MP2/6-31+G(d) & 30.8 & 9.0 & 27.9 & 18.4 & 22.0 & 26.8 \\
MP2/6-31G(d) & 26.5 & 5.0 & 23.0 & 15.0 & 18.5 & 23.0 \\
B3LYP/6-311++G(3df,2p) & 39.6 & 20.3 & 37.1 & 27.3 & 32.0 & 34.5 \\
B3LYP/6-311G(3df,2p) & 39.4 & 19.2 & 36.4 & 27.3 & 31.7 & 34.7 \\
B3LYP/6-311++G(d) & 28.6 & 9.3 & 27.0 & 17.0 & 21.3 & 24.0 \\
B3LYP/6-31+G(2d,p) & 35.0 & 15.8 & 32.8 & 23.2 & 27.5 & 30.4 \\
B3LYP/6-31G(2d) & 32.2 & 14.1 & 29.4 & 21.5 & 25.5 & 28.3 \\
B3LYP/6-31G(d,p) & 28.4 & 7.7 & 25.9 & 16.7 & 20.7 & 24.4 \\
B3LYP/6-311G(d) & 28.4 & 7.5 & 25.9 & 16.8 & 20.9 & 24.2 \\
B3LYP/6-31+G(d) & 29.0 & 9.4 & 27.4 & 17.2 & 21.5 & 24.6 \\
B3LYP/6-31G(d) & 27.8 & 7.9 & 25.3 & 16.5 & 20.5 & 23.9 \\
G3 (0 K) & 22.9 & 22.8 & 38.5 & 30.9 & 34.6 & 38.2 \\
G3 (298 K) & 17.8 & 35.0 & 23.8 & 30.3 & 34.5 \\
\hline
\end{tabular}

${ }^{\mathrm{a}}$ All BDE were determined from the listed method energies performed at the MP2/6-31G(d,p) geometry and include the unscaled ZPE and temperature correction to $298.15 \mathrm{~K}$. Calculations are for the direct dissociation reactions, not an isodesmic reaction. 
Table S5. Calculated BDEs for sulfonium carbenes $\mathbf{1 9 - 2 3}$ in $\mathrm{kcal} / \mathrm{mol}$.

\begin{tabular}{|c|c|c|c|c|c|}
\hline Method $^{\mathrm{a}}$ & 19 & 20 & 21 & 22 & 23 \\
\hline MP2/6-311++G(3df,2p) & 45.8 & 26.5 & 33.7 & 38.4 & 43.6 \\
\hline MP2/6-311G(3df,2p) & 44.9 & 25.6 & 32.9 & 37.5 & 43.1 \\
\hline MP2/6-311++G(d) & 35.4 & 18.1 & 25.7 & 29.4 & 33.6 \\
\hline MP2/6-31+G(2d,p) & 37.7 & 18.5 & 25.7 & 30.2 & 35.6 \\
\hline MP2/6-31G(2d) & 33.9 & 16.5 & 23.1 & 27.4 & 33.1 \\
\hline MP2/6-31G(d,p) & 29.5 & 12.4 & 18.9 & 23.4 & 28.4 \\
\hline MP2/6-311G(d) & 34.1 & 16.8 & 20.8 & 27.9 & 32.7 \\
\hline MP2/6-31+G(d) & 32.6 & 14.6 & 21.4 & 26.2 & 30.8 \\
\hline MP2/6-31G(d) & 29.0 & 12.5 & 18.8 & 23.3 & 28.0 \\
\hline B3LYP/6-311++G(3df,2p) & 40.4 & 24.7 & 29.4 & 34.7 & 37.2 \\
\hline B3LYP/6-311G(3df,2p) & 40.1 & 24.5 & 29.4 & 34.5 & 37.4 \\
\hline B3LYP/6-311++G(d) & 34.1 & 19.6 & 23.6 & 29.1 & 31.3 \\
\hline $\mathrm{B} 3 \mathrm{LYP} / 6-31+\mathrm{G}(2 \mathrm{~d}, \mathrm{p})$ & 37.1 & 21.8 & 26.5 & 31.7 & 34.2 \\
\hline B3LYP/6-31G(2d) & 35.4 & 22.2 & 26.0 & 31.0 & 33.5 \\
\hline B3LYP/6-31G(d,p) & 33.3 & 17.9 & 22.8 & 27.8 & 31.3 \\
\hline B3LYP/6-311G(d) & 34.0 & 19.5 & 23.7 & 28.9 & 31.7 \\
\hline B3LYP/6-31+G(d) & 56.4 & 41.3 & 45.7 & 51.1 & 53.5 \\
\hline B3LYP/6-31G(d) & 33.2 & 18.7 & 23.1 & 28.2 & 31.2 \\
\hline G3 (0 K) & 44.6 & 27.3 & 33.6 & 38.0 & 42.1 \\
\hline G3 (298 K) & 40.6 & 22.3 & 29.1 & 33.6 & 38.1 \\
\hline
\end{tabular}

\footnotetext{
${ }^{a}$ All BDE were determined from the listed method energies performed at the MP2/6-31G(d,p) geometry and include the unscaled ZPE and temperature correction to $298.15 \mathrm{~K}$. Calculations are for the direct dissociation reactions, not an isodesmic reaction.
} 
Table S6. Computed BDEs for selenoxides 27-32, in $\mathrm{kcal} / \mathrm{mol}$

\begin{tabular}{|c|c|c|c|c|c|c|}
\hline Method $^{\mathrm{a}}$ & 27 & 28 & 29 & 30 & 31 & 32 \\
\hline MP2/6-311++G(3df,2p) & 79.1 & 75.7 & 74.6 & 57.5 & 59.8 & 64.2 \\
\hline MP2/6-311G(3df,2p) & 78.5 & 75.1 & 74.4 & & & \\
\hline MP2/6-311++G(d) & 62.3 & 59.6 & 58.8 & & & \\
\hline MP2/6-31+G(2d,p) & 80.0 & 71.1 & 75.0 & & & \\
\hline MP2/6-31G(2d) & 74.4 & 71.7 & 71.4 & & & \\
\hline MP2/6-31G(d,p) & 64.7 & 61.2 & 62.5 & & & \\
\hline MP2/6-311G(d) & 57.2 & 54.4 & 54.2 & & & \\
\hline MP2/6-31+G(d) & 74.5 & 71.1 & 69.5 & & & \\
\hline MP2/6-31G(d) & 64.8 & 61.4 & 62.9 & & & \\
\hline B3LYP/6-311++G(3df,2p) & 69.6 & 68.5 & 65.5 & & & \\
\hline B3LYP/6-311G(3df,2p) & 70.3 & 69.3 & 61.0 & & & \\
\hline B3LYP/6-311++G(d) & 61.6 & 61.0 & 57.9 & & & \\
\hline B3LYP/6-31+G(2d,p) & 73.4 & 72.3 & 68.4 & & & \\
\hline B3LYP/6-31G(2d) & 70.4 & 69.9 & 67.4 & & & \\
\hline B3LYP/6-31G(d,p) & 65.4 & 64.0 & 62.9 & & & \\
\hline B3LYP/6-311G(d) & 59.1 & 58.6 & 55.9 & & & \\
\hline B3LYP/6-31+G(d) & 71.2 & 70.0 & 65.9 & & & \\
\hline B3LYP/6-31G(d) & 65.2 & 64.0 & 62.9 & & & \\
\hline G3 (0 K) & 71.5 & 67.6 & 68.4 & & & \\
\hline G3 & 70.2 & 68.9 & 66.3 & & & \\
\hline
\end{tabular}

${ }^{a}$ All $\Delta \mathrm{H}$ were determined from the listed method energies runs performed at the MP2/6-31G $(\mathrm{d}, \mathrm{p})$ geometry and include the unscaled ZPE and a $298.15 \mathrm{~K}$ temperature correction. Calculations for 27-29 are for the direct dissociation reactions, not an isodesmic reaction. The entries for 30-32 corresponds to Method C. 